\title{
Genome-wide locus sequence typing (GLST) of eukaryotic pathogens
}

Philipp Schwabl ${ }^{\mathrm{a}}$, Jalil Maiguashca Sánchez ${ }^{\mathrm{b}}$, Jaime A. Costales ${ }^{\mathrm{b}}$, Sofía Ocaña ${ }^{\mathrm{b}}$, Maikell Segoviac, Hernán J. Carrasco $^{\mathrm{c}}$, Carolina Hernández ${ }^{\mathrm{d}}$, Juan David Ramírez ${ }^{\mathrm{d}}$, Michael D. Lewis ${ }^{\mathrm{e}}$, Mario J. Grijalva ${ }^{\mathrm{b}, \mathrm{f}}$ and Martin S. Llewellyn $^{\mathrm{a}}$

${ }^{a}$ Institute of Biodiversity, Animal Health \& Comparative Medicine, University of Glasgow, Glasgow G12 8QQ, UK

${ }^{\mathrm{b}}$ Centro de Investigación para la Salud en América Latina, Pontificia Universidad Católica del Ecuador, Quito, Ecuador

'Laboratorio de Biología Molecular de Protozoarios, Instituto de Medicina Tropical, Universidad Central de Venezuela, Caracas, Venezuela

${ }^{\mathrm{d}}$ Grupo de Investigaciones Microbiológicas, Programa de Biología, Universidad del Rosario, Bogotá, Colombia

${ }^{\mathrm{e}}$ London School of Hygiene \& Tropical Medicine, Keppel Street, London, WC1E 7HT, UK

fInfectious and Tropical Disease Institute, Biomedical Sciences Department, Heritage College of Osteopathic Medicine, Ohio University, 45701 Athens, OH, USA

\section{Abstract}

Analysis of genetic polymorphism is a powerful tool for epidemiological surveillance and research. Powerful inference from pathogen genetic variation, however, is often restrained by limited access to representative target DNA, especially in the study of obligate parasitic species for which ex vivo culture is resource-intensive or bias-prone. Modern sequence capture methods enable pathogen genetic variation to be analyzed directly from vector/host material but are often too complex and expensive for resource-poor settings where infectious diseases prevail. This study proposes a simple, cost-effective 'genome-wide locus sequence typing' (GLST) tool based on massive parallel amplification of information hotspots throughout the target pathogen genome. The multiplexed polymerase chain reaction amplifies hundreds of different, user-defined genetic targets in a single reaction tube, and subsequent agarose gel-based clean-up and barcoding completes library preparation at under 4 USD per sample. Approximately 100 libraries can be sequenced together in one Illumina MiSeq run. Our study generates a flexible GLST primer panel design workflow for Trypanosoma cruzi, the parasitic agent of Chagas disease. We successfully apply our 203-target GLST panel to direct, culture-free metagenomic extracts from triatomine vectors containing a minimum of $3.69 \mathrm{pg} / \mu 1$ T. cruzi DNA and further elaborate on method performance by sequencing GLST libraries from $T$. cruzi reference clones representing discrete typing units (DTUs) TcI, TcIII, TcIV, and TcVI. The 780 SNP sites we identify in the sample set repeatably distinguish parasites infecting sympatric vectors and detect correlations between genetic and geographic distances at regional $(<150 \mathrm{~km})$ as well as continental scales. The markers also clearly separate DTUs. We discuss the advantages, limitations and prospects of our method across a spectrum of epidemiological research. 


\section{Introduction}

Genome-wide single nucleotide polymorphism (SNP) analysis is a powerful and increasingly common approach in the study and surveillance of infectious disease. Understanding patterns of SNP diversity within pathogen genomes and across pathogen populations can resolve fundamental biological questions (e.g., reproductive mechanisms in $T$. cruzi ${ }^{1}$, reconstruct past ${ }^{2}$ and present transmission networks (e.g., Staphylococcus infections within hospitals) ${ }^{3}$ or identify the genetic bases of virulence ${ }^{4,5}$ and resistance to drugs (see examples from Plasmodium spp. ${ }^{6,7}$ ). A number of obstacles, however, complicate access to representative, genome-wide SNP information using modern sequencing tools. Micro-pathogens are often sampled in low quantities and together with large amounts of host/vector tissue, microbiota, or environmental DNA. Sequencing is rarely viable directly from the infection source and studies have often found it necessary to isolate and culture the target organism to higher densities before extracting DNA. These additional steps, however, are resource-intensive and bias-prone. Pathogen isolation is less often attempted on asymptomatic infections and is less likely to succeed when levels of parasitaemia in a sample are low. Genomic sequencing data on the protozoan parasite Leishmania infantum, for example, has for such reasons come to exhibit major selection bias towards aggressive strains isolated by invasive sampling from canine hosts. A short look into the limited number of whole-genome sequencing (WGS) datasets available for L. infantum at the European Nucleotide Archive (ENA) quickly confirms this statement. Vector-isolated genomes have yet to be reported from the Americas and only a single study claims to have sequenced L. infantum from asymptomatic hosts ${ }^{8}$. Selection bias also often occurs due to competition among isolated strains. Studies on the kinetoplastid Trypanosoma cruzi, for example, are time and again confounded by growth and survival rate differences among genotypes in culture ${ }^{9-11}$, and gradual reductions to genetic diversity are often observed over time ${ }^{12}$. Karyotypic changes are also known to arise during T. cruzi micromanipulation and axenic growth ${ }^{13,14}$.

A variety of approaches therefore aim to obtain genome-wide SNP information without first performing pathogen isolation and culturing steps. Some studies separate target sequences from total DNA or RNA by exploiting base modifications or transcriptional properties specific to the pathogen ${ }^{15}$, vector ${ }^{16}$ or host $^{17,18}$. Others describe the use of biotinylated hybridization probes ${ }^{19-22}$ or selective whole-genome amplification, e.g., based on the strand displacement function of phi29 DNA polymerase ${ }^{23}$. Such techniques are costly and often excessive when a study's primary objective is to evaluate genetic distances and diversity among samples rather than to reconstruct complete haplotypes or investigate structural genetic traits. Epidemiological tracking and source attribution studies, for example, often benefit little from measuring invariant sequence areas or defining the complete architecture of sample genomes. Also pathogen typing or population assignment objectives primarily require information on polymorphic sites. It is nevertheless quite common to see such studies to undertake expensive WGS procedures only for final analyses to take place 'post-VCF' ${ }^{24}$, i.e., using a list of diagnostic markers compiled from a small fraction of polymorphic reads.

Highly multiplexed polymerase chain reaction (PCR) amplicon sequencing offers a much more efficient option when obtaining genome-wide SNP information is the primary goal. First marketed under the name Ion 
AmpliSeq by Thermo Fisher Scientific ${ }^{25}$, the method consists in the simultaneous amplification of dozens to hundreds of DNA targets known or hypothesized to contain sequence polymorphism in the sample set. Each sample's resultant amplicon pool is then prepared for sequencing by index/adaptor ligation or in a subsequent 'barcoding' PCR. Panel construction is highly flexible, requiring only that the primers exhibit similar melting/annealing temperatures and a low propensity to cross-react. As such, target selection can be tailored to specific research goals, for example, to profile resistance markers ${ }^{26}$ or to genotype neutral SNP variation for landscape genetic techniques ${ }^{27}$. The potential to isolate and genotype pathogen DNA at high-resolution directly from uncultured sample types by multiplexed amplicon sequencing has however received little attention thus far. Simultaneous PCR-based detection of multiple pathogen species or genotypes is certainly $\operatorname{common}^{28}$, but multiplexable primer panels are rarely designed for subsequent sequencing and polymorphism analysis. The Ion AmpliSeq brand currently offers pre-designed panels for studies on ebola ${ }^{29}$ and tuberculosis ${ }^{30}$ but the use of custom panels for other pathogen species (e.g., Bifidobacterium ${ }^{31}$ or human papilloma virus ${ }^{32}$ ) remains surprisingly rare in the literature.

In this study we describe the design and implementation of a large multiplexable primer panel for $T$. cruzi, parasitic agent of Chagas disease. In contrast to past multi-locus sequence typing (MLST) methods involving at most 32 (individually amplified) gene fragments, our 'genome-wide locus typing' (GLST) tool simultaneously amplifies 203 sequence targets across 33 (of 47) T. cruzi chromosomes. We apply GLST to metagenomic DNA extracts from triatomine vectors collected in Colombia, Venezuela and Ecuador and further describe method sensitivity/specificity by sequencing GLST libraries from $T$. cruzi clones representing discrete typing units (DTUs) TcI, TcIII, TcIV, and TcVI. The 780 SNP sites identified from GLST amplicon sequencing repeatably distinguish parasites infecting sympatric vectors and detect correlations between genetic and geographic distances at regional $(<150 \mathrm{~km})$ and continental scales. The markers also clearly separate DTUs. We discuss the advantages and limitations of our method for epidemiological studies in resource-poor settings where Chagas and other 'neglected tropical diseases' prevail.

\section{Methods}

\section{Triatomine samples and $T$. cruzi reference clones}

T. cruzi-infected intestinal tract and/or faeces samples of Rhodnius ecuadoriensis and Panstrongylus chinai were collected by the Centro de Investigación para la Salud en América Latina (CISeAL) in Loja Province, Ecuador, following protocols described in Grijalva et al. 2012 ${ }^{33}$. DNeasy Blood and Tissue Kit (Qiagen) was used to extract metagenomic DNA. Infected intestinal material of Panstrongylus geniculatus, $R$. pallescens and $R$. prolixus from northern Colombia was also collected in previous projects ${ }^{34-36}$, likewise using DNeasy Blood and Tissue Kit to extract metagenomic DNA. Panstrongylus geniculatus specimens from Caracas, Venezuela were collected by the citizen science triatomine collection program (http://www.chipo.chagas.ucv.ve/vista/index.php) at Universidad Central de Venezuela. This program has supported various epidemiological studies in the capital district ${ }^{37-39}$. DNA was extracted from the insect faeces 
by isopropanol precipitation. Geographic coordinates and ecotypes (domestic, peri-domestic, or sylvatic) of the sequenced samples are provided in Supplementary Tbl. 1.

T. cruzi epimastigote DNA from reference clones Chile c22 (TcI) Arma18 cl. 1 (TcIII), Saimiri3 cl. 8 (TcIV), Para7 cl. 3 (TcVI), Chaco9 col. 15 (TcVI) and CL Brener (TcVI) was obtained from the London School of Hygiene \& Tropical Medicine (LSHTM). DNA extractions at LSHTM followed Messenger et al. $2015^{40}$.

Uninfected Rhodnius prolixus gut tissue samples used for mock infections (see 'Method development and library preparation') were also provided by LSHTM. Special thanks to C. Whitehorn and M. Yeo for supervising dissections. Insects were euthanized with $\mathrm{CO}_{2}$ and hindguts drawn into 5 volumes of RNAlater (Sigma-Aldrich) by pulling the abdominal apex toward the posterior with sterile watchmaker's forceps.

T. cruzi TcI X10/1 Sylvio reference clone ('TcI-Sylvio') epimastigotes used for mock infections and various other stages of method development were obtained from CISeAL. Cryo-preserved cells were returned to logphase growth in liver infusion tryptose (LIT) and quantified by hemocytometer before pelleting at 25,000 g. Pellets were washed twice in PBS and parasites killed by resuspension in 10 volumes of RNAlater. DNA from these T. cruzi cells (and their dilutions with preserved T. prolixus intestinal tissue) was extracted by isopropanol precipitation.

Isopropanol precipitation was also used to extract DNA from T. cruzi plate clone TBM_2795_CL2. This sample was previously analyzed by WGS ${ }^{1}$ and served as a control for GLST method development in this study.

\section{GLST target and primer selection}

We began our GLST sequence target selection process by screening single-nucleotide variants previously identified in T. cruzi populations from southern Ecuador ${ }^{1}$. Briefly, Schwabl et al. sequenced genomic DNA from 45 cloned and 14 non-cloned T. cruzi field isolates on the Illumina HiSeq 2500 platform and mapped resultant $125 \mathrm{nt}$ reads to the TcI-Sylvio reference assembly using default settings in BWA-mem v0.7.3 ${ }^{41}$. Single-nucleotide polymorphisms (SNPs) were summarized by population-based genotype and likelihood assignment in Genome Analysis Toolkit v3.7.042, excluding sites with low cumulative call confidence (QUAL $<1,500)$ and/or aberrant read-depth $(<10$ or $>100)$ as well as those belonging to clusters of three or more SNPs. A 'virtual mappability' mask ${ }^{43}$ was also applied to avoid SNP inference in areas of high sequence redundancy in the $T$. cruzi genome. Read-mapping and variant exclusion criteria were verified by subjecting TcI-Sylvio Illumina reads from Franzen et al. $2012^{44}$ to the same pipelines as the Ecuadorian dataset. An additional mask was set around small insertion-deletions suggested to occur in these reads based on the assumption that the reference sample should not present alternate genotypes in high-quality contigs of the assembled genome.

We extracted $160 \mathrm{nt}$ segments from the T. cruzi reference genome (.fasta file) whose internal sequence (positions 41 to 120) contained between one and ten of 75,038 SNPs identified in the above WGS dataset. 
These 56,428 segments were further filtered for synteny between T. cruzi and Leishmania major genomes as defined by the OrthoMCL algorithm at TriTrypDB ${ }^{45}$. Such conserved segments may be least prone to repeatdriven nucleotide diversity and as such most amenable to $\mathrm{PCR}^{46}$. The 6,259 synteny segments found by OrthoMCL therefore proceeded to primer search with the high-throughput primer design engine BatchPrimer $3^{47}$. As target SNPs did not occur in the outer $40 \mathrm{nt}$ of each synteny segment, these flanking regions provided additional flexibility to identify primers matching the following criteria:

- $\quad$ min. size $=24 \mathrm{nt}$

- $\quad \max$. size $=35 \mathrm{nt}$

- $\quad$ optimal size $=24 \mathrm{nt}$

- $\quad$ min. product size $=120 \mathrm{nt}$

- $\quad$ max. product size $=160 \mathrm{nt}$

- $\quad$ optimal product size $=120 \mathrm{nt}$

- $\quad$ min. melting temperature $=63^{\circ} \mathrm{C}$,

- $\quad$ max. melting temperature $=65^{\circ} \mathrm{C}$,

- optimal melting temperature $=63{ }^{\circ} \mathrm{C}$,

- max. self-complementarity: $4 \mathrm{nt}$

- $\quad \max .3$ ' self-complementarity: $2 \mathrm{nt}$

- $\quad$ max. length of mononucleotide repeats $=3 \mathrm{nt}$

- $\min$. GC content $=40 \%$

- $\max$. GC content $=60 \%$

Each of 286 forward primer candidates output by BatchPrimer3 received the additional 5' tag sequence 5'ACACTGACGACATGGTTCTACA-3' and reverse primer candidates received the 5' tag sequence 5'TACGGTAGCAGAGACTTGGTCT-3'. These tag sequences enable single-end barcode and Illumina P5/P7 adaptor attachment in second-round PCR. Next, we determined binding energies $(\Delta G)$ for all possible primerpairs using the primer compatibility software MultiPLX v2.1.4. We discarded primers with inter-quartile ranges crossing a threshold of $\Delta \mathrm{G}=-12.0 \mathrm{kcal} / \mathrm{mol}$. Primers with 20 or more interactions showing $\Delta \mathrm{G} \leq-12.0$ $\mathrm{kcal} / \mathrm{mol}$ were also disallowed. The remaining 248 primer-pairs (median $\Delta \mathrm{G}=-9.0$ ) underwent a last filtering step by screening for perfect matches in raw WGS sequence files (.fastq). Low match frequency led to the elimination of 45 additional primer pairs. WGS alignments corresponding to the 203 sequence regions targeted by this final primer set were visualized in Belvu v12.4.3 ${ }^{48}$. The 403 SNPs occurring within these sequence regions distributed evenly across individuals in Loja Province. Using the 'nj' function from the 'ape' package v5.0 in R v3.4. ${ }^{49}$, the 403 SNPs also reproduced neighbor-joining relationships observed based on total polymorphism identified by WGS (Supplementary Fig. 1). These observations lent further support to the suitability of the GLST marker panel for the analysis of genetic differentiation at the landscape-scale. The GLST sequence target selection process described above is summarized in Fig. 1. 
bioRxiv preprint doi: https://doi.org/10.1101/2020.03.24.003590; this version posted March 25, 2020. The copyright holder for this preprint (which was not certified by peer review) is the author/funder, who has granted bioRxiv a license to display the preprint in perpetuity. It is made available under aCC-BY-ND 4.0 International license.

181

182

183

184

185

186

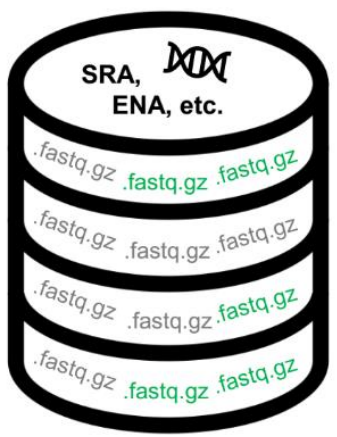

9) Simulate prospective GLST-based analyses, e.g., by abridging the WGS-VCF to include only target SNPS and comparing $\mathrm{NJ}$ relationships with those obtained from the unabridged WGS-VCF. It is also useful to visualize target alignments, e.g., to ensure that outlier samples have not mislead marker choice (unless prior MAF filters can exclude such effects).
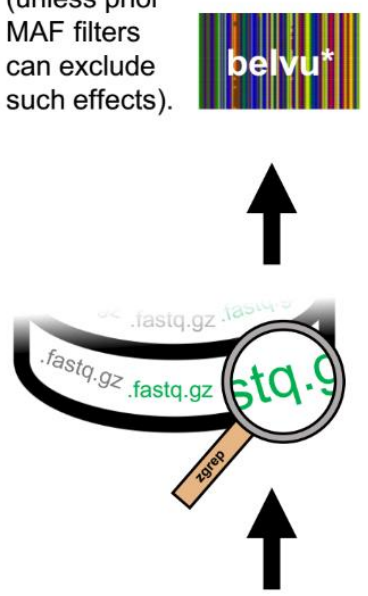

8) Exclude primer sequences that do not occur in WGS reads (also search for reverse complements).

7) Calculate primer alignment energies $(\Delta G)$, e.g., using MultiPLX*. Remove primers with high non-target affinity, e.g., primers for which $\Delta G$ inter-quartile ranges cross $-12.0 \mathrm{kcal} / \mathrm{mol}^{*}$.

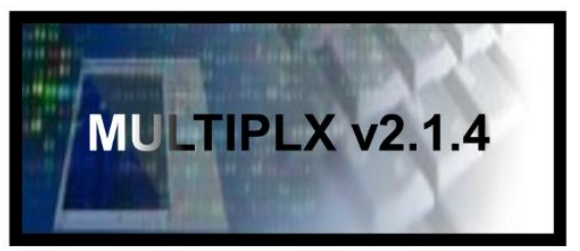

Repeat after removing cross-reactive primers to assess final $\Delta \mathrm{G}$ scores.

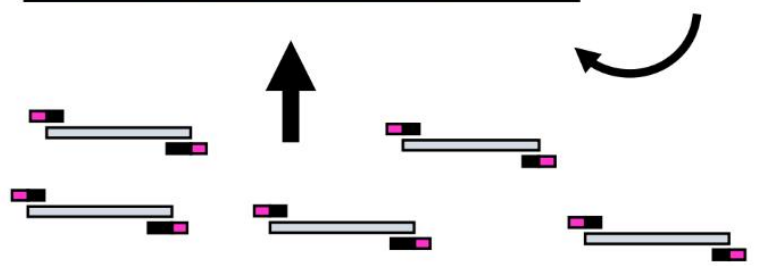

6) Add 5' tags (ㅁ) to primers ( $\square$ ) from Batchprimer3.

These $22 \mathrm{nt}^{\star}$ tags contain binding sites for barcoding PCR.
2) Identify SNP variants in mapped WGS reads (GATK* ${ }^{*}$ FreeBayes, SAMtools, etc). Summarize in VCF.
1) Map WGS reads from public database (or pilot study) to reference genome

(BWA*, SMALT, Bowtie, etc.)

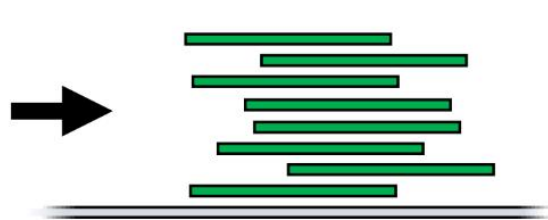

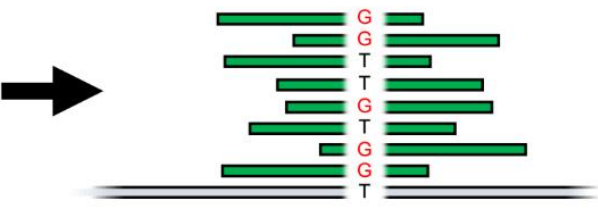

3) Apply SNP quality control (e.g., GATK best practices) and filter for amenability to PCR and amplicon mapping, e.g., by selecting for 'virtually mappable** or single-copy sequence regions ${ }^{\star}$ ). Also apply study-specific filters (e.g., restrict levels of linkage or MAF) to arrive at preliminary set of candidate SNPs.
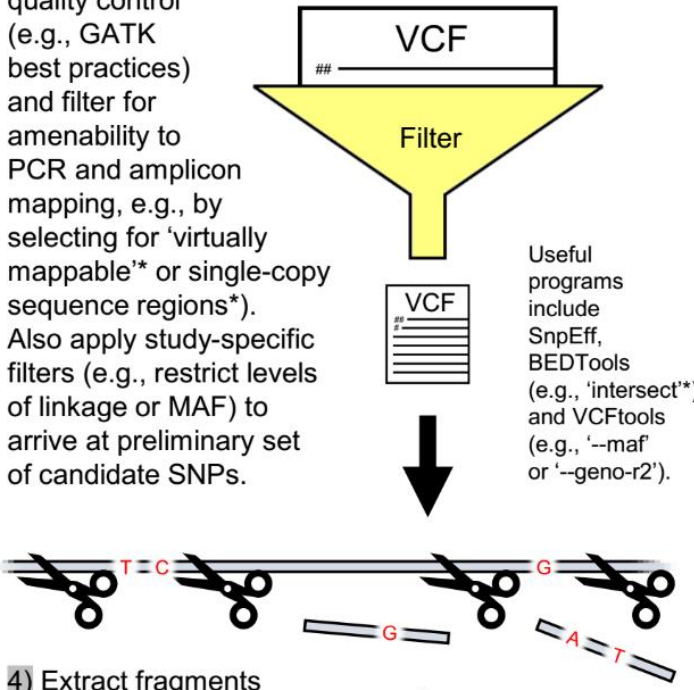

4) Extract fragments from reference genome where candidate SNPs occur. Ensure SNPs do not occur near fragment edges (e.g., within $40 \mathrm{nt}^{\star}$ ).

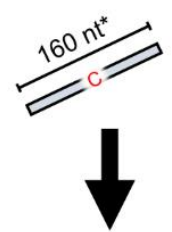

Useful programs include VCFtools '--SNPdensity"* and SAMtools 'faidx'.

\section{BatchPrimer3 v1.0}

5) Submit fragments to primer design software, e.g., Batchprimer $3^{\star}$. Key design parameters include min. / max. / opt. primer size, e.g., 24 / 35 / 24 nt*, $^{*}$

min. / max. / opt. product size, e.g., $120 / 160 / 120$ nt $^{*}$,

min. / max. / opt. melting temp., e.g., $63 / 65 / 63^{\circ} \mathrm{C}^{*}$,

max. self-complementarity, e.g., $4 \mathrm{nt}^{*}$, max. 3' self-complementarity, e.g., $2 \mathrm{nt}^{*}$, max. length mononuc. repeats, e.g., $3 \mathrm{nt}^{*}$, and $\mathrm{min}$. / max. GC content, e.g., $40 / 60 \%$ *
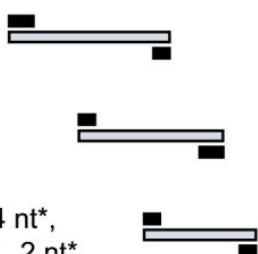

Figure 1 GLST sequence target selection from preliminary genomic data. Nine steps of primer panel construction and validation run clockwise from top left. Various methods and criteria can be applied to complete many of these steps. Those specific to this study are asterisked, e.g., we used BWA in step 1 and GATK in step 2. Abbreviations: SRA (Sequence Read Archive at www.ncbi.nlm.nih.gov/sra); ENA (European Nucleotide Database at www.ebi.ac.uk/ena; WGS (whole-genome sequencing); SNP (single-nucleotide polymorphism); MAF (minor allele frequency); PCR (polymerase chain reaction); VCF (variant call format); NJ (neighbor-joining). 


\section{Wet lab method development and library preparation}

The 203 primers pairs designed above (Supplementary Tbl. 2) were purchased from Eurofins Genomics (Ebersberg, Germany) at $200 \mu \mathrm{M}$ concentration in salt-free, 96-well plate format. Primer pairs were first tested individually to establish cycling conditions for PCR (Supplementary Fig. 2). Optimal target amplification occurred with an initial incubation step at $98{ }^{\circ} \mathrm{C}(2 \mathrm{~min}) ; 30$ amplification cycles at $98{ }^{\circ} \mathrm{C}(10 \mathrm{~s}), 60{ }^{\circ} \mathrm{C}(30 \mathrm{~s})$, and $72{ }^{\circ} \mathrm{C}(45 \mathrm{~s})$; and a final extension step at $72{ }^{\circ} \mathrm{C}(2 \mathrm{~min})$. The $10 \mu \mathrm{l}$ reactions contained $5 \mu \mathrm{l}$ Q5 HighFidelity Master Mix (New England Biolabs), $1 \mu \mathrm{l}$ forward primer $[10 \mu \mathrm{M}], 1 \mu 1$ reverse primer $[10 \mu \mathrm{M}]$, and $3 \mu 1$ TcI-Sylvio epimastigote DNA. The multiplexed, first-round 'GLST' PCR reaction was prepared by combining all 406 primers in equal proportions and diluting the combined mix to $50.75 \mu \mathrm{M}$, resulting in individual primer concentrations of $50.75 \mu \mathrm{M} / 406=125 \mathrm{nM}$. GLST reactions incorporated $2 \mu \mathrm{l}$ of this primer mix rather than two separate $1 \mu \mathrm{l}$ forward/reverse primer inputs as above.

We first tested GLST PCR on DNA extracts from mock infections, each consisting of $10^{4}, 10^{5}$ or $10^{6}$ TcISylvio epimastigote cells and one uninfected R. prolixus intestinal tract (Supplementary Fig. 3). Amplicons from lower concentration epimastigote dilutions gave weaker signals in gel electrophoresis, suggesting lower infection load thresholds at which vector gut DNA becomes unsuitable for GLST. Most vector gut DNA extracts obtained for this study represented donated material of limited quality and infection load, some samples were also without signal in PCR spot tests for the presence of high frequency ' $\mathrm{TcZ}$ ' ${ }^{50}$ satellite DNA (commonly targeted to diagnose human T. cruzi infections).

We therefore first used qPCR to identify vector gut samples containing $T$. cruzi DNA quantities within ranges successfully visualized from GLST reactions on epimastigote DNA quantified by Qubit fluorometry (Invitrogen) and serially diluted from $1.35 \mathrm{ng} / \mu \mathrm{l}$ to $2.50 \mathrm{pg} / \mu \mathrm{l}$ in $\mathrm{dH}_{2} \mathrm{O}$ (Supplementary Fig. 4). Each $20 \mu \mathrm{l}$ qPCR reaction consisted of $10 \mu$ l SensiMix SYBR Low-ROX reagent (Bioline), $1 \mu 1$ TcZ forward primer $\left(5^{\prime} \text {-GCTCTTGCCCACAMGGGTGC-3') }\right)^{50} \quad[10 \quad \mu \mathrm{M}], \quad 1 \quad \mu l \quad$ TcZ $\quad \begin{array}{llll}10 & \text { reverse primer }\end{array}$

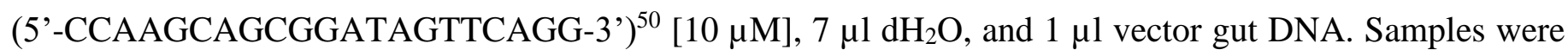
amplified together with a 15 -step standard curve containing between $0.30 \mathrm{pg}$ and $4.82 \mathrm{ng} T$. cruzi epimastigote DNA. Reaction conditions consisted of an initial incubation step at $95^{\circ} \mathrm{C}(10 \mathrm{~min})$ and 40 amplification cycles at $95{ }^{\circ} \mathrm{C}(15 \mathrm{~s}), 55{ }^{\circ} \mathrm{C}(15 \mathrm{~s})$, and $72{ }^{\circ} \mathrm{C}(15 \mathrm{~s})$. Fluorescence acquisition occurred at the end of each cycle and final product dissociation was measured in $0.5^{\circ} \mathrm{C}$ increments between 55 and $95{ }^{\circ} \mathrm{C}$.

Vector gut samples suggested to contain at least $1.0 \mathrm{pg} / \mu 1 \mathrm{~T}$. cruzi concentrations based on qPCR proceeded to final library construction (Supplementary. Tbl. 1) alongside DNA from T. cruzi clones TBM_2795_cl2 (TcI), Chile c22 (TcI) Arma18 cl. 1 (TcIII), Saimiri3 cl. 8 (TcIV), Para7 cl. 3 (TcV), Chaco9 col. 15 (TcVI) and CL Brener (TcVI). Several samples were processed in $2-4$ replicates beginning with the first-round GLST PCR reaction step. First-round PCR products were electrophoresed in $0.8 \%$ agarose gel to separate target bands (mode $=164 \mathrm{nt}$ ) from primer polymers quantified with the Agilent Bioanalyzer 2100 System (see $78 \mathrm{nt}$ primer peak in Supplementary Fig. 5). Excised target bands were resolubilized with the PureLink Quick 
Gel Extraction Kit (Invitrogen) to create input for subsequent barcoding PCR. This second PCR reaction consisted of an initial incubation step at $98{ }^{\circ} \mathrm{C}(2 \mathrm{~min}) ; 7$ amplification cycles at $98{ }^{\circ} \mathrm{C}(30 \mathrm{~s}), 60{ }^{\circ} \mathrm{C}(30 \mathrm{~s})$, and $72{ }^{\circ} \mathrm{C}(1 \mathrm{~min})$; and a final extension step at $72{ }^{\circ} \mathrm{C}(3 \mathrm{~min})$. Only 7 amplification cycles were used given polymer 'daisy-chaining' observed when cycling at 13 and 18x (Supplementary Fig. 6). The barcoding reaction adds Illumina flow cell and sequencing primer binding sites to each first-round PCR product. A different reverse primer is used for each sample. The reverse primer (5'-CAAGCAGAAGACGGCATACGAGAT*X*TACGGTAGCAGAGACTTGGTCT-3') contains a $10 \mathrm{nt}$ barcode $\left(* X^{*}\right)$ to distinguish reads from different samples during pooled sequencing. It also contains CS2 (sequencing primer binding sites). A single forward primer (5'-AATGATACGGCGACCACCGAGATCTACACTGACGACATGGTTCTA-3') containing CS1 is used for all samples. Each $20 \mu \mathrm{l}$ barcoding reaction contained $10 \mu \mathrm{l}$ Q5 High-Fidelity Master Mix (New England Biolabs), $0.8 \mu \mathrm{l}$ forward (universal) primer [10 $\mu \mathrm{M}$ ], $0.8 \mu \mathrm{l}$ (barcoded) reverse primer [10 $\mu \mathrm{M}], 5.4 \mu 1 \mathrm{dH}_{2} \mathrm{O}$ and $3 \mu \mathrm{l}$ (gel-purified) first-round PCR product. Barcoding primers were purchased from Eurofins Genomics at $100 \mu \mathrm{M}$ concentration in HPLC-purified, 96-well plate format. Barcoded amplicons (e.g., Supplementary Fig. 7) were quantified by Qubit fluorometry (Thermo Fisher Scientific), and pooled at equimolar concentrations, gel-excised, re-solubilized, and verified by microfluidic electrophoresis (Supplementary Fig. 8) as above.

\section{GLST amplicon sequencing and variant discovery}

The GLST pool was sequenced twice on an Illumina MiSeq instrument. We first used the pool to 'spike' additional base diversity into a collaborator's $16 \mathrm{~S}$ amplicon sequencing run. 16S samples were loaded to achieve $80 \%$ sequence output whereas GLST and PhiX DNA ${ }^{51}$ were each loaded at $10 \%$. This first run occurred in 500-cycle format using MiSeq Reagent Kit v2. The second run occurred in 300-cycle format using MiSeq Reagent Micro Kit v2 and was dedicated solely to GLST (also no PhiX). Both runs were performed at Glasgow Polyomics using Fluidigm Custom Access Array sequencing primers FL1 (CS1 + CS2) and CS2 rc $^{52}$.

Demultiplexed sequence reads were trimmed to $120 \mathrm{nt}$ and mapped to the TcI-Sylvio reference assembly using default settings in BWA-mem v0.7.3. Mapped reads with poor alignment scores (AS < 100) were discarded to decontaminate samples of non-T.cruzi sequences sharing barcodes with the GLST dataset. Identical results were achieved using BWA-sw in DeconSeq v0.4.3 $3^{53}$ to decontaminate reads. After merging alignment (.bam) files from sequencing runs 1 and 2 with Picard Tools v1.11 ${ }^{54}$, single-nucleotide polymorphisms (SNPs) were identified in each sample using the 'HaplotypeCaller' algorithm in GATK v3.7.0 ${ }^{42}$. Population-based genotype and likelihood assignment followed using 'GenotypeGVCFs'. We excluded SNP sites with QUAL $<$ 80, D < 10, Mapping Quality (MQ) < 80 and or Fisher Strand Bias (FS) > 10. Individual genotypes were set to missing (./.) if they contained $<10$ reads and set to reference $(0 / 0)$ if they contained only a single alternate read (i.e., if they were classified as heterozygotes based on minor allele frequencies $\leq 10 \%$ ). These filtering thresholds were cleared by all expected SNPs (i.e., SNPs also found in prior WGS sequencing) but not by all new SNPs found using GLST (e.g., see comparison of QUAL density curves in Supplementary Fig. 9). SNP 
calling with GATK was also performed separately for sequencing runs 1 and 2 in order to exclude SNP sites uncommon to both analyses from the merged dataset described above.

\section{GLST repeatability, population genetic and spatial analyses}

We used PopART v1.7 to plot genetic differences between samples and sample replicates as a median-joining network, i.e., a minimum spanning tree composed of observed sequences and unobserved (reconstructed) sequence nodes ${ }^{55}$. Genetic differences were measured by applying the 'vcf-to-tab' script from VCFtools v0.1.13 to the filtered SNP dataset, concatenating each sample's output fields and counting the number of mismatching alleles $(0,1$ or 2$)$ per site and sample pair. A phylogenetic tree was built by counting the number of non-reference alleles in each genotype with the VCFtools function '--012', summing pairwise Euclidean distances at biallelic sites and plotting neighbor-joining relationships with the 'nj' function from the 'ape' package v5.0 in $\mathrm{R}$ v3.4.199.

Considering only the first replicate of multiply sequenced samples, linkage and neutrality statistics were calculated using VCFtools functions '--geno-r2' (calculates correlation coefficients between genotypes following Purcell et al. ${ }^{56}$ ), '--het' (calculates inbreeding coefficients using a method of moments ${ }^{57}$ ) and '-hwe' (filters sites by deviation from Hardy-Weinberg Equilibrium following Wigginton et al. ${ }^{58}$ ). FST differentiation was calculated using ARLSUMSTAT v3.5.2 $2^{59,60}$.

Correlations between geographic and genetic differences were also calculated from non-reference allele counts in R v3.4.1 ${ }^{49}$. The 'mantel' function from the 'vegan' package v2.4.4 ${ }^{61}$ was used to test significance of the Mantel statistic by permuting geographic distances and re-measuring correlations to genetic distances 999 times. Again, we used only the first replicate for samples with replicate sets. DTU reference clones were also excluded from analysis. Geographic distances were measured by projecting sample latitude/longitude (WGS 84) coordinates into a common xy plane (EPSG code 3786) selected following Šavrič et al. $2016^{62}$ (Supplementary Tbl. 1). EPSG 3786 projection was also used to map samples with the Natural Earth quick start kit in QGIS v2.18.4.

Given that missing information in sequence alignment can confound inference on genetic distances between samples ${ }^{63}$, above repeatability and phylogenetic analyses excluded SNP sites in which genotypes were missing for any individual, and mantel analyses excluded SNP sites in which genotypes were missing in $>10 \%$ individuals. These exclusion criteria initially led to significant information loss due to the presence of two outlier samples, ARMA18_CL1_rep2 and COL253, libraries of which had been sequenced despite poor target visibility in gel electrophoresis (i.e., final PCR product banding appeared similar to that of ECU2 in Supplementary Fig. 7). Read-depths for the two samples ended up averaging 1.2 interquartile ranges below the sample set median and precluded genotype assignment at $>25 \%$ SNP sites. We therefore decided to exclude them from all analyses. 


\section{Results}

\section{SNP polymorphism and repeatability}

GLST amplicons contained a total of 780 SNP sites, 387 polymorphic among TcI samples and 393 private to non-TcI reference clones (Fig. 2). Median read-depth was 266x across all sites. Of 403 loci targeted from the WGS dataset $^{1}, 97 \%$ (391) were recovered by GLST and 82 contained polymorphism outside of Ecuador. GLST recovered 80 of 87 SNPs previously identified in TBM_2795_CL2 using WGS. Minimum parasite DNA concentration successfully genotyped from metagenomic DNA was $3.69 \mathrm{pg} / \mu 1$ (sample ECU36 - see Supplementary Fig. 10).

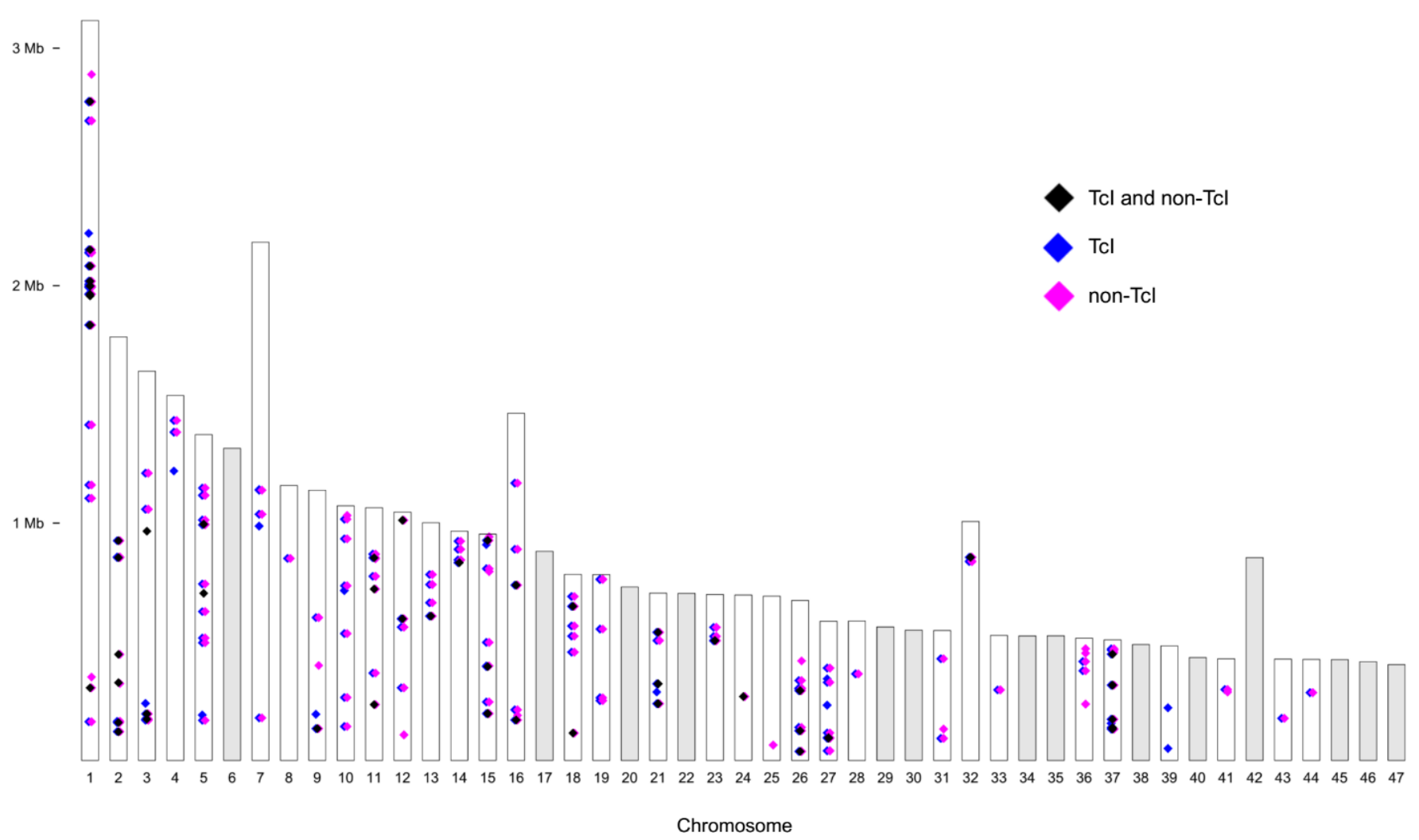

Figure 2 Variant loci detected in T. cruzi I samples and reference clones of other sub-lineages. The genome-wide distribution of SNP variants is shown relative to the Tcl-Sylvio reference assembly. Each column represents one of 47 putative chromosomes. Pink diamonds comprise 393 variants that occur only in non-Tcl samples. The remaining 387 variants are private to (blue) or shared by $\mathrm{Tcl}$ and other sub-lineages (black). Diamonds representing nearby SNPs (e.g., those occurring on the same GLST target segment) overlap at this scale.

The TBM_2795_CL2 control sample underwent GLST in four replicates. These replicates were identical at all 561 SNP sites for which genotypes were called in all samples of the dataset. Median number of allelic differences ( $\mathrm{AD}=0,1$ or 2 per site) at non-missing sites between other replicate pairs was 3 (Tbl. 1). Pairwise $\mathrm{AD}$ did not correlate to minimum, maximum or difference in mean read-depth between the two replicates ( $\mathrm{p}$ $<0.80)$. 
bioRxiv preprint doi: https://doi.org/10.1101/2020.0324.003590; this version posted March 25, 2020. The copyright holder for this preprint (which was not certified by peer review) is the author/funder, who has granted bioRxiv a license to display the preprint in perpetuity. It is made available under aCC-BY-ND 4.0 International license.

Read-mapping coverage was inconsistent among replicates but strongly correlated between sequencing runs (Pearson's $r=0.93, p<0.001$ ) (Supplementary Figs. $11-12$ ). Variant calling was also highly consistent: prior to variant filtration, only 10 SNP sites were called from run1 that were not also called from run 2 (these were excluded from analysis - see Methods).

\section{Differentiation among $T$. cruzi individuals, sampling areas and sub-lineages}

Sampling sites in Colombia, Venezuela and Ecuador are plotted in Fig. 3, and a median-joining network of allelic differences among GLST genotypes is shown in Fig. 4. GLST clearly distinguished TcI individuals at common collection sites in Soata (COL466 vs. COL468, AD = 37), Paz de Ariporo (COL133 vs. COL135, $\mathrm{AD}=33$ ), Tamara (COL154 vs. COL155 AD = 107) and Lebrija (COL77 vs. COL78, $\mathrm{AD}=43$ ) municipalities of Colombia but not in the community of Bramaderos (ECU3 vs. ECU8 vs. ECU10, AD =0) in Loja Province, Ecuador. Samples from nearby sites within Caracas, Venezuela were also clearly distinguished by GLST (e.g., VZ16816 vs. VZ17114, AD = 43).

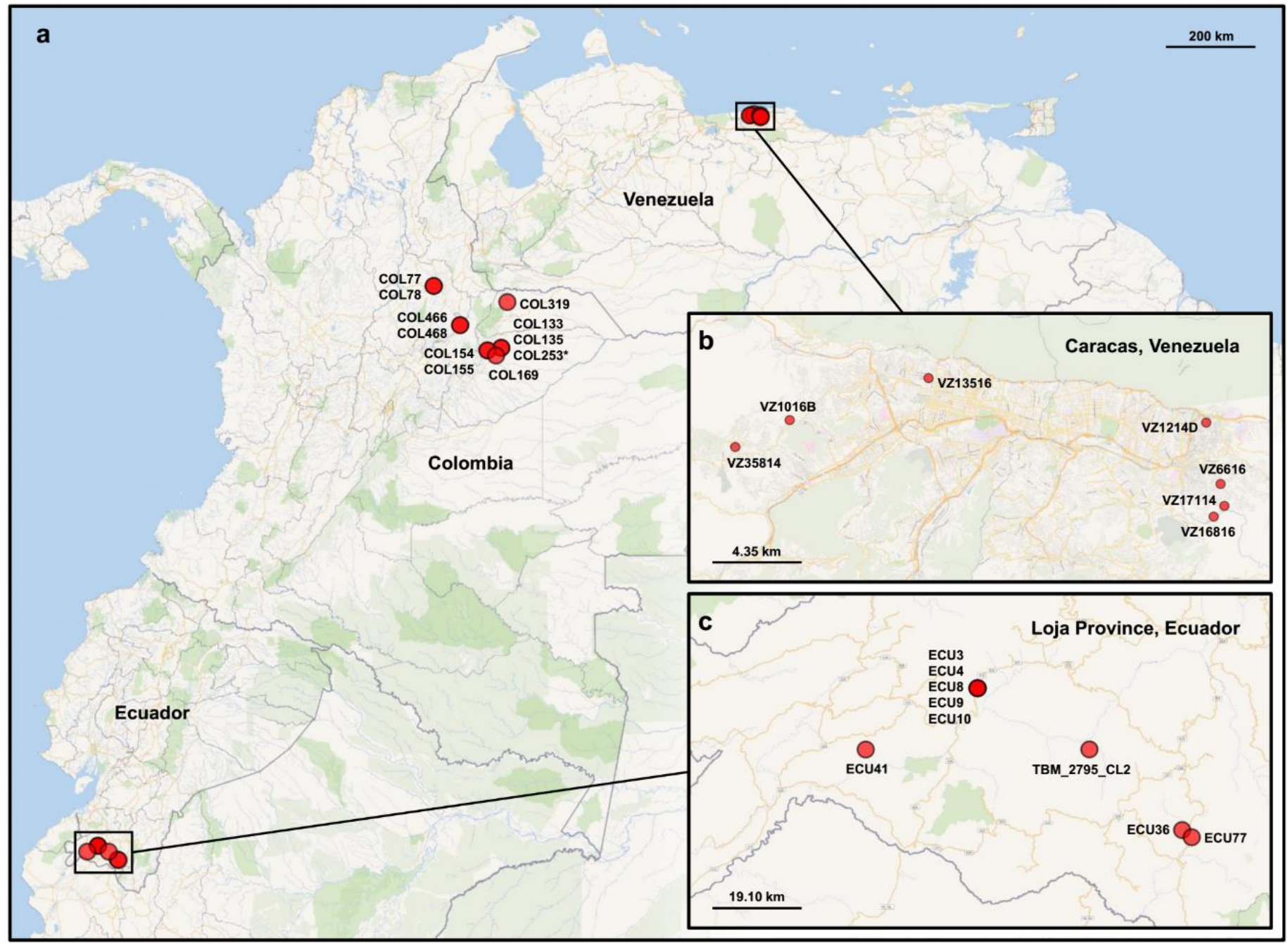

Figure 3 Map of vector sampling sites. a Sampling in Colombia involved a larger spatial area than that in Venezuela and Ecuador. T. cruzi-infected intestinal material was collected from Panstrongylus and Rhodnius vectors in Arauca, Casanare, Santander and Boyacá. We asterisk COL253 because low read-depth led to sample exclusion. b $P$. geniculatus material from Venezuela was collected within the Metropolitan District of Caracas. c $R$. ecuadoriensis and $P$. chinai material from Ecuador was collected in Loja Province. Supplementary Tbl. 1 lists coordinates and other details. 
387 Nucleotide diversity ( $\pi=$ mean pairwise AD) was higher in samples from Caracas $(\pi=29.0)$ than in those from Loja Province $(\pi=22.8)$ but not in those from Colombia $(\pi=43.2)$ (Tbl. 2$)$. Hardy-Weinberg ratios, linkage and inbreeding coefficients are also listed in Tbl. 2.

\section{Tcl Colombia}
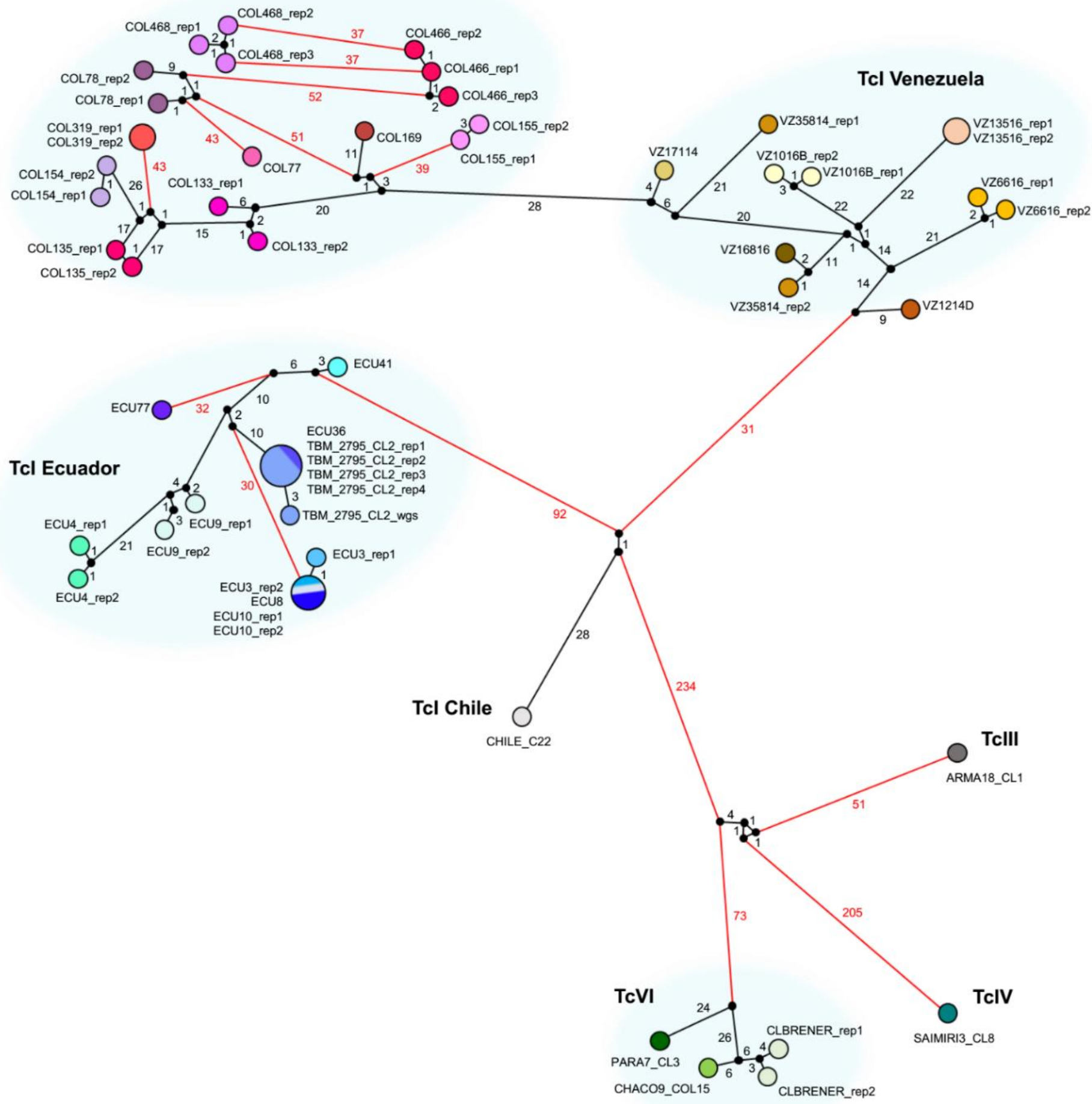

Figure 4 Allelic differences among T. cruzi I samples and reference clones of other sub-lineages as a median-joining network. A single SNP locus can differ by 0,1 or 2 between two individuals (i.e., the individuals match at both, one, or neither allele). The $A D$ measurement indicated on each edge of the network represents the total number of differences across all loci for which genotypes were called in all individuals of the dataset $(n=561)$. Red edges indicate differences of 30 and above. Technical replicates are represented by circles of the same fill color. Larger circles represent the occurrence of identical GLST genotypes. Edge length is not directly proportional to AD. 
Table 1 Allelic differences between GLST replicates. Eighteen samples were processed in $2-4$ replicates after DNA extraction. A single SNP locus can differ by 0,1 or 2 between two replicates (i.e., replicates can match at both, one, or neither allele). The AD measurement represents the total number of pairwise differences across all loci for which genotypes are called in all individuals $(\mathrm{n}=$ 561). The discrepancy between VZ35814 replicates likely represents barcode contamination with VZ16816 (see close similarity in Fig. 3).

\section{Replicate comparison}

\section{AD}

COL319_rep1 vs. COL319_rep2

ECU10_rep1 vs. ECU10_rep2

TBM_2795_CL2_rep1 vs. TBM_2795_CL2_rep2

TBM_2795_CL2_rep1 vs. TBM_2795_CL2_rep3

TBM_2795_CL2_rep1 vs. TBM_2795_CL2_rep4

TBM_2795_CL2_rep2 vs. TBM_2795_CL2_rep3

TBM_2795_CL2_rep2 vs. TBM_2795_CL2_rep4

TBM_2795_CL2_rep3 vs. TBM_2795_CL2_rep4

VZ13516_rep1 vs. VZ13516_rep2

COL154_rep1 vs. COL154_rep2

COL466_rep1 vs. COL466_rep2

ECU3_rep1 vs. ECU3_rep2

COL135_rep1 vs. COL135_rep2

COL468_rep1 vs. COL468_rep2

ECU4_rep1 vs. ECU4_rep2

COL155_rep1 vs. COL155_rep2

COL466_rep1 vs. COL466_rep3

COL468_rep1 vs. COL468_rep3

COL468_rep2 vs. COL468_rep3

VZ6616_rep1 vs. VZ6616_rep2

COL466_rep2 vs. COL466_rep3

VZ1016B_rep1 vs. VZ1016B_rep2

CL_Brener_rep1 vs. CL_Brener_rep2

COL133_rep1 vs. COL133_rep2

ECU9_rep1 vs. ECU9_rep2

COL78_rep1 vs. COL78_rep2

VZ35814_rep1 vs. VZ35814_rep2

0
0
0
0
0
0
0
0
0
1
1
1
2
2
2
2
3
3
3
3
3
3
3
3
4
3

49

Table 2 Basic diversity statistics for T. cruzi I samples from Colombia (COL), Venezuela (VZ) and Ecuador (ECU). Abbreviations: n (sample size); PS (polymorphic sites); HWE (Hardy-Weinberg equilibrium); $F_{I S}$ (inbreeding coefficient), $r^{2}$ (linkage coefficient), $\Pi$ (nucleotide diversity), $Q$ (quartile); $M$ (median); Fst (between-group fixation index).

$\begin{array}{rcccccccc}\text { Group (n) } & \text { PS } & \begin{array}{c}\text { PS in } \\ \text { HWE }\end{array} & \text { Fis (Q1, M, Q3) } & \mathbf{r}^{2}(\mathbf{Q 1}, \mathbf{M}, \mathbf{Q 3}) & \mathbf{\pi} & \begin{array}{c}\mathbf{F s T} \\ \text { to COL }\end{array} & \begin{array}{c}\text { Fst } \\ \text { to VZ }\end{array} & \begin{array}{c}\text { Fst } \\ \text { to ECU }\end{array} \\ \text { COL (11) } & 175 & 169 & -0.19,0.13,0.24 & 0.03,0.07,0.19 & 43.2 & 0.000 & 0.136 & 0.595 \\ \text { VZ (7) } & 147 & 143 & -0.35,-0.19,0.11 & 0.02,0.09,0.27 & 29.0 & 0.136 & 0.000 & 0.632 \\ \text { ECU (9) } & 148 & 142 & -0.20,-0.09,0.18 & 0.04,0.17,0.36 & 22.8 & 0.595 & 0.632 & 0.000\end{array}$


Genetic distances increased with spatial distances among samples (Mantel's $r=0.89, p=0.001$ ), but the correlation coefficient was largely driven by high FST between sample sets from Colombia/Venezuela and Ecuador (Tbl. 2 and Fig. 5a): Mantel's $r$ decreased to $0.30(p=0.001)$ after restricting analysis to sample pairs separated by $<250 \mathrm{~km}$ (Fig. 5b). Within-country IBD appeared to grow stronger for samples separated by < $150 \mathrm{~km}$ (Mantel's $r=0.48, p=0.002$ ) given a lack of correlation observed at higher distance classes within the Colombian dataset (Fig. 5b).

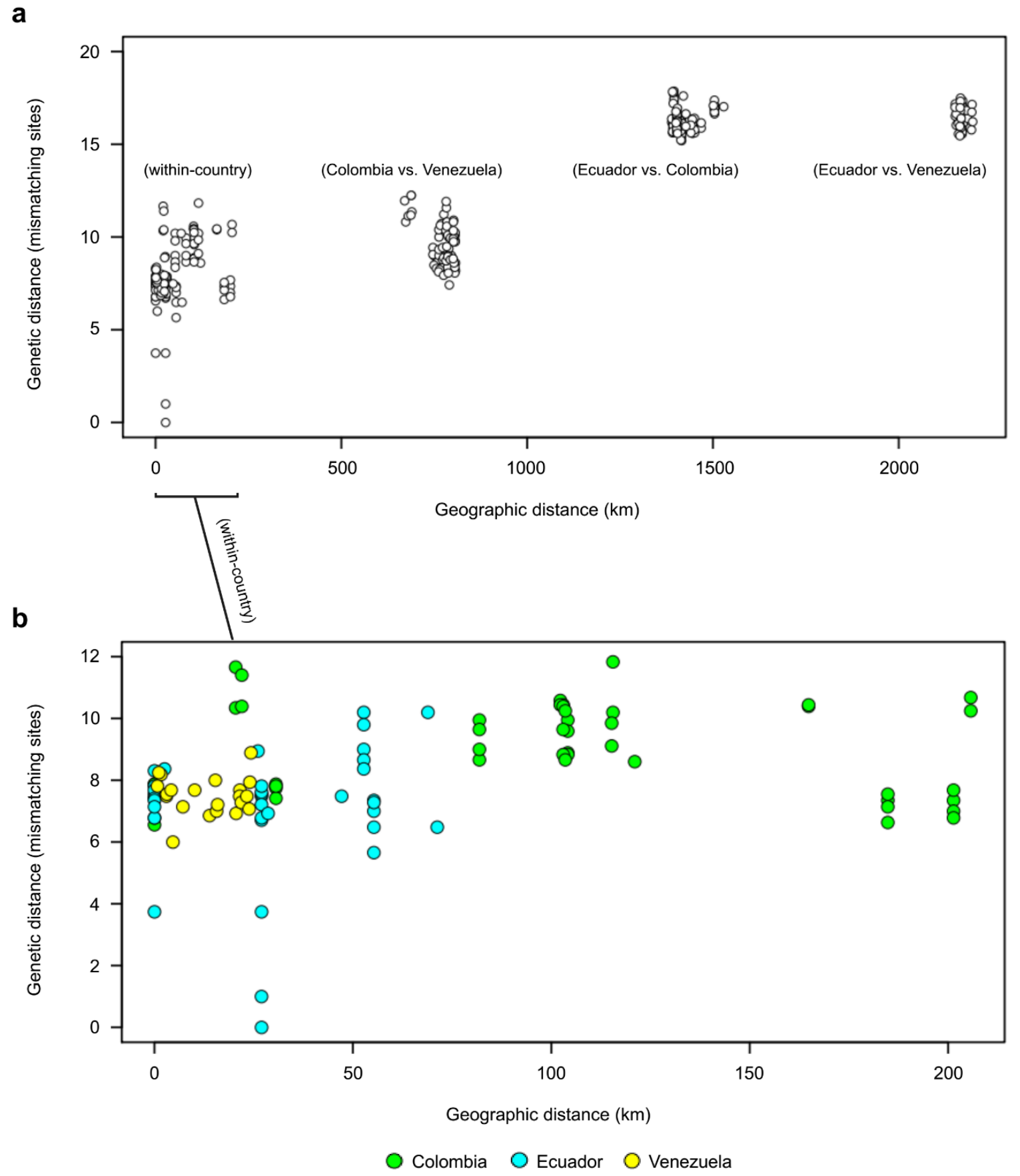

Figure 5 Isolation-by-distance among T. cruzi I samples. a Each circle represents geographic and genetic distances between two Tcl samples. Global isolation-by-distance (IBD) is significant (Mantel's $r=0.89, p=0.001$ ) but driven by divergence between Ecuadorian samples and the rest of dataset (see two clusters at top right). b Nevertheless, IBD remains significant for within-country comparisons at $<250 \mathrm{~km}$ (Mantel's $r=0.30, p=0.009$ ) and $<150 \mathrm{~km}$ (Mantel's $r$ $=0.48, p=0.002)$. Green, cyan and yellow fill colors represent comparisons within Colombia, Ecuador and Venezuela, respectively. Each of the above Mantel tests remains significant when sample pairs with genetic distances $<2$ are removed (see arrows). Only variant sites with $\leq 10 \%$ missing genotypes $(n=285)$ are used in analysis. Only the first replicate is used for samples represented by multiple replicates. 
481 Finally, GLST also clearly separated sub-lineages TcI, TcIII, TcIV, and TcVI in network (Fig. 3) and neighbor-joining tree construction (Fig. 6). AD between reference clones of different sub-lineages ranged from 153 (Arma18 cl1 (TcIV) vs. Para7 cl.3 (TcV)) to 472 (Chile c22 (TcI) vs. Saimiri3 cl. 8 (TcIV)).

Tcl Colombia

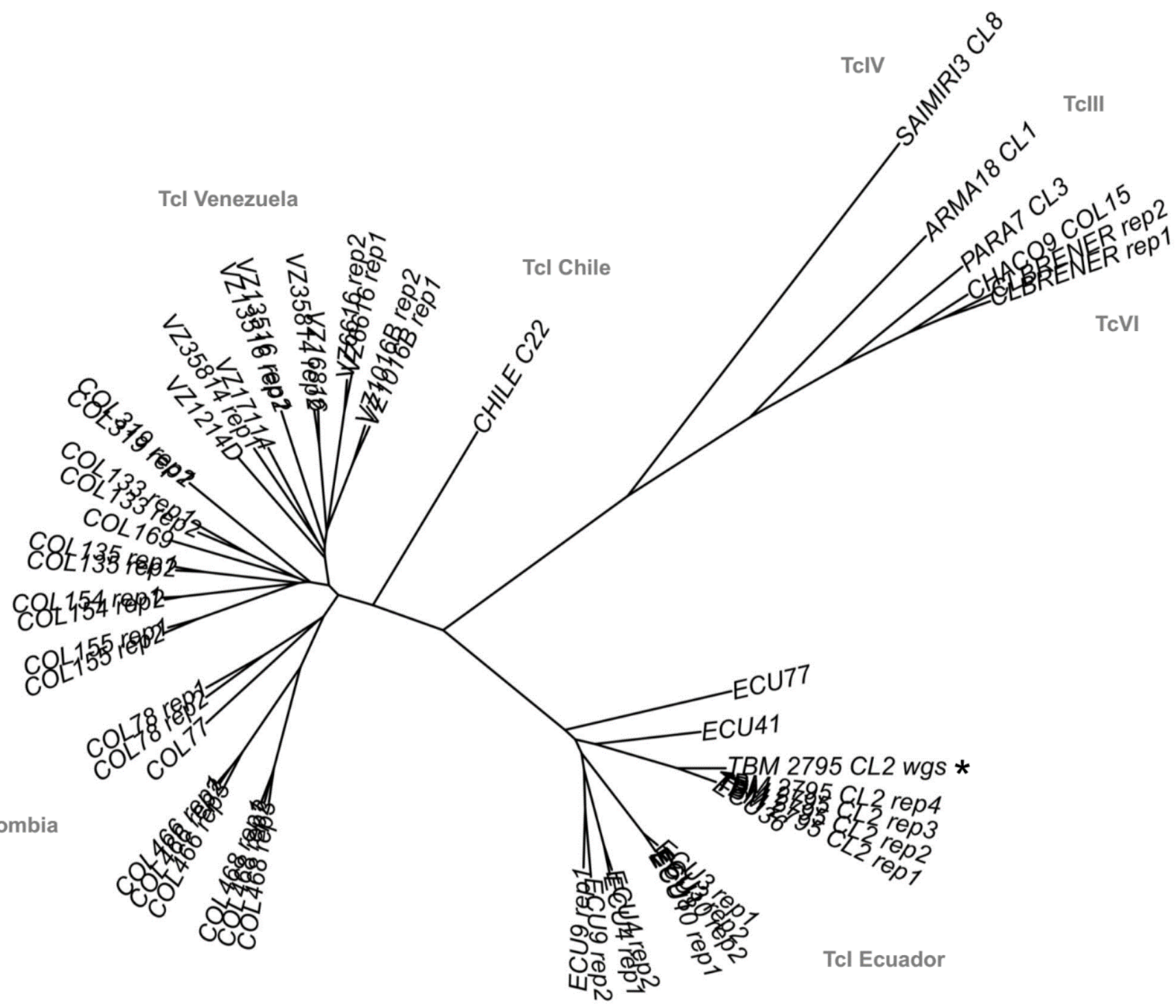

Figure 6 Neighbor-joining relationships among T. cruzi I samples and reference clones of other sub-lineages. Genetic distances are based on 556 biallelic SNP sites for which genotypes are called in all individuals. Results indicate high repeatability among most technical replicates (see 'rep1 - 4' suffices) and clearly separate Tcl, Tclll, TcIV and TcVI. The tree also contains TBM_2795_CL2_wgs (see asterisk). This control sample was genotyped at the same 556 GLST loci using whole-genome sequencing (Illumina HiSeq) data from Schwabl et al. $2019^{1}$. 


\section{Discussion}

\section{Principle results}

The GLST primer panel design and amplicon sequencing workflow outlined in this study aimed to profile $T$. cruzi genotypes at high resolution directly from infected triatomine intestinal content by simultaneous amplification of 203 genetic target regions that display sequence polymorphism in publicly available WGS reads. Mapped GLST amplicon sequences generated from $T$. cruzi reference clones and from metagenomic intestinal DNA extracts containing a minimum of $3.69 \mathrm{pg} / \mu \mathrm{l}$ T. cruzi DNA achieved high target specificity $(<$ $1 \%$ off-target mapping) and yield (391 of 403 target SNP sites mapped). Mapping depth variation across target loci was highly repeatable between sequencing runs. 387 SNP sites were identified among T. cruzi DTU I samples and 393 SNP sites were identified in non-TcI reference clones. These markers showed low linkage and clearly separated T. cruzi individuals within and across DTUs, for the most part also individuals collected at the same or closely separated localities in Colombia, Venezuela, and Ecuador. An increase in pairwise genetic differentiation was observed with increasing geographic distance in analyses within and beyond 150 $\mathrm{km}$.

\section{Cost-effective spatio-genetic analysis}

GLST achieved an important resolution benchmark in recovering isolation-by-distance (IBD) ${ }^{64}$ at less than $150 \mathrm{~km}$. These correlations indicate the potential of GLST in spatially explicit epidemiological studies which, for example, aim to identify environmental variables or landscape features that modify IBD ${ }^{27}$. High spatial sampling effort is typically required by such studies and often limits budget for genotyping tools. GLST appears promising in this context as library preparation costs $<4.00$ USD per sample (see cost summary in Supplementary Tbl. 3) and can be completed comfortably in two days. The first-round PCR reaction requires very low primer concentrations $(0.125 \mu \mathrm{M})$ such that a single GLST panel purchase $(0.01 \mu$ mol production scale) enables $>100,000$ reactions and can be shared by several research groups. Sequencing represents a substantial cost but is highly efficient due to short fragment sizes and few off-target reads. High library complexity also promotes the use of GLST in the role of PhiX, i.e., as a spike-in to enhance read quality in a different sequencing run. Our study easily decontaminated reads from a spiked amplicon pool sharing barcodes with GLST (run 1). Alternatively, i.e, when GLST is sequenced alone (run 2), one Illumina MiSeq run is expected to generate $>70 \mathrm{x}$ median genotype depth for 100 samples using Reagent Micro Kit v2 (ca. 1,000 - 1,500 USD, depending on provider; Supplementary Tbl. 3).

\section{GLST in relation to multi-locus microsatellite typing}

We consider multi-locus microsatellite typing (MLMT) as the primary alternative for high-resolution T. cruzi genotyping directly from metagenomic DNA. MLMT has revolutionized theory on T. cruzi ecology and microevolution, for example, on the role of disparate transmission cycles ${ }^{65,66}$, ecological host-fitting ${ }^{67}$ and 'cryptic sexuality ${ }^{68}$ in shaping population genetic structure in TcI. In some cases ${ }^{69,70}$ (but others not ${ }^{66,67,71}$ ), the hypervariable, multiallelic nature of microsatellites allows every sample in a dataset to be distinguished 
with a different multi-locus genotype (MLG). This depends on panel size and spatial scale but also on local reproductive modes - e.g., sampling from clonal sylvatic vs. non-clonal domestic transmission cycles has correlated with the presence or absence of repeated MLGs ${ }^{66}$. In this study, we found two identical GLST genotypes shared among five samples from southern Ecuador. All other samples appeared unique, including those from Venezuela, where triatomine collection occurred at seven domestic localities within the city of Caracas. The small subset of repeated genotypes found in this study may reflect patchy, transmission cycledependent clonal/sexual population structure in southern Ecuador (see Schwabl et al. $2019^{1}$ and OcañaMayorga et al. 2010 ${ }^{66}$ ) but may also represent a weakness in GLST compared to MLMT in tracking individual parasite strains. The use of large MLMT panels, however, is significantly more resource-intensive because each microsatellite marker requires a separate PCR reaction and capillary electrophoresis cannot be highly multiplexed. MLMT data are poorly archivable across studies and may also be less suitable for inter-lineage phylogenetic analyses due to unclear mutational models and artefactual similarity from saturation effects ${ }^{72}$. Although our GLST panel was designed for TcI, its focus on syntenous sequence regions enabled efficient coamplification of non-TcI DNA. GLST clearly separated TcI samples from all non-TcI reference clones, with highest divergence observed in Saimiri3 cl. 8. Interestingly, large MLMT panels have shown comparatively little differentiation between this sample and TcI, also more generally suggesting that TcIV and TcI represent monophyletic sister clades ${ }^{72}$.

\section{Adjustment and transferability}

Considering the great variety of sample types to which studies have successfully applied PCR ${ }^{73-77}$, we expect that GLST can be applied to metagenomic DNA from many host/vector tissue types, not only from triatomine intestine as shown here. Further tests are required to determine whether low T. cruzi DNA concentrations in chronic infections or sparsely infected organs (e.g., liver and heart ${ }^{78}$ ) are also amenable to GLST. We focused analysis on $T$. cruzi DNA concentrations of at least one picogram per microliter metagenomic DNA (this equates to ca. 30 parasites per microliter in the case of $\mathrm{TcI}^{79}$ ) without heavily investigating options to enhance sensitivity or sensitivity measurement, for example, by additional removal of PCR inhibitors, improved primer purification (e.g., HPLC vs. salt-free), post-PCR probe-hybridization ${ }^{80}$ or barcoding/sequencing of samples with unclear first-round PCR amplicon bands. Even relatively aggressive processing methods may be tolerable given that DNA fragmentation is unlikely to compromise the 120 - $160 \mathrm{nt}$ size range targeted by GLST. Increasing sensitivity by increasing PCR amplification cycles, however, is less advised. PCR error appeared relevant with as little as 30x (+7x barcoding) amplification in this study as we observed noise among replicates despite high read-depth and SNP-call overlap between sequencing runs. Rates or error were, however, well within margins expected for methods involving $\mathrm{PCR}^{81}$. We also note that the exceptional discrepancy between VZ35814 replicates unlikely represents systematic error but barcode contamination with VZ16816. Such error is perhaps less likely if primers are kept in separate vials instead of in the plate format which we have used here. 
Wet lab aside, the main objective of this study was to provide a transparent bioinformatic workflow for highly multiplexable primer panel design using freely available softwares and publicly archived WGS reads (e.g., see www.ebi.ac.uk/ena or www.ncbi.nlm.nih.gov/sra). Importantly, we show that knowledge of polymorphic genetic regions in parasite genomes from one small study area (Loja Province, Ecuador) can suffice to guide variant discovery at distant, unassociated sampling sites. Our demonstration using T. cruzi should be easily transferable to any other pathogenic species with a published reference genome. Target selection can also be tailored to a variety of objectives. For example, while landscape genetic studies on dispersal often focus on neutral or non-coding sequence variation ${ }^{82}$, experimental (e.g., drug testing) studies may seek to detect singlenucleotide changes in coding regions, perhaps in genes belonging to specific ontology groups or associated with results of high-throughput proteomic screens ${ }^{83}$. The candidate SNP pool can easily be filtered for such criteria during GLST panel design, e.g., using SnpEff $^{84}$ or BEDTools ${ }^{85}$ and data mining strategies at EuPathDB ${ }^{86}$. Candidate SNP filtering by minor allele frequency (MAF) may also be useful when the target population is closely related to that of the WGS dataset guiding panel design. Placing a minimum threshold on MAF (using VCFtools ${ }^{87}$, etc.), for example, may improve analyses of population structure and genealogy whereas a focus on low-frequency variants may help in tracking individuals or recent gene flow at the landscape scale ${ }^{88}$. It may also be possible to refine panel design towards markers that meet model assumptions in later analysis. Hardy Weinberg Equilibrium (HWE), for example, is a common requirement in demographic modelling ${ }^{89-91}$, Bayesian clustering ${ }^{92}$, admixture/migration ${ }^{93,94}$ and hybridization tests ${ }^{95}$. Deviation from HWE may occur more frequently in specific genetic regions (e.g., near centromeres ${ }^{96}$ ), and SNPs in these could be excluded from the target pool. Numerous other filtering options - e.g., based on allele count (to enhance resolution per SNP), distance to insertion-deletions (to improve target alignment), or percent missing information (to avoid poorly mapping regions) - are easily implemented with common analysis tools ${ }^{97}$.

GLST is also highly scalable because increasing panel size does not lead to more laboratory effort or processing time. Sequencing depth requirements and thermodynamic compatibilities among primers are more relevant in limiting panel size. However, it is also possible to divide large GLST panels into two or more PCR multiplexes based on $\Delta$ G-based partitioning in MultiPLX ${ }^{98}$. Unintended primer affinities (i.e., polymer formations) can also be removed by gel excision, e.g., as we have done using the PureLink Quick Gel Extraction Kit.

\section{Prospects}

This study sought to provide a framework for various epidemiological research but was restricted in its own ability to make important inferences on $T$. cruzi ecology because only few samples (remainders from different projects) were analyzed. Samples were also aggregated either to domestic or to sylvatic ecotopes (see Supplementary Tbl. 1). More extensive, purposeful sampling could have, for example, helped us explore whether COL468's position deep within the Cordillera Oriental contributes to its strong divergence to samples such as COL135 or COL319, these perhaps more closely related due to lower 'cost-distances' 99 along the basin range. Fuelling landscape genetic simulators such as $\mathrm{CDMetaPOP}^{91}$ with high GLST sample sizes is an 
especially exciting direction for future research. It would also be interesting, for example, to extend this study's sampling to cover gradients along the perimeter of Caracas and adjacent El Ávila National Park (see Fig. 4b). Sylvatic $P$. geniculatus vector populations appear to be rapidly adapting to habitats within Caracas $^{39,100}$ but parallel changes in the distribution of $T$. cruzi genetic diversity have yet to be tracked. The low cost of GLST also makes it more feasible for studies to simultaneously assess genetic polymorphism in each vector individual from which parasite markers were amplified. Such coupled genotyping would enhance resolution of parasite-vector genetic co-structure and thus, for example, help quantify rates of parasite transmission from domiciliating vectors or determine whether parasite gene flow proxies for (or improves understanding of) dispersal patterns in more slowly evolving vectors or hosts. It would also be interesting to test in how far deep-sequenced GLST libraries could help in detecting (and reconstructing distinct MLGs from) multiclonal T. cruzi infections without the use of cloning tools ${ }^{101}$, e.g., using bioinformatic strategies developed for malaria research ${ }^{102-105}$. Multiclonality has important implications for public health ${ }^{106,107}$ but its potential prevalence in $T$. cruzi vectors and hosts ${ }^{108,101,109}$ is difficult to describe from cultured cells ${ }^{108,110}$. Countless other applications are conceivable for GLST. Some research fields, however, will surely be less amenable to the PCR-based approach. Relative amplicon concentrations, for example, appeared to be too stochastic in this study to allow inference of copy number variation or other structural rearrangements based on read-mapping depths. Unintended primer alignment is also likely to occur if PCR targets are located within highly repetitive sequences such as those encoding surface protein families in sub-telomeric regions of the $T$. cruzi genome ${ }^{46}$.

We look forward to seeing GLST approaches in a wide variety of research for which such limitations do not apply. Regarding population and landscape genetic studies, prudent spatial and genetic sampling design is often key to meaningful inference and we hope that the low cost and high flexibility of our pipeline helps researchers achieve all criteria required. 
bioRxiv preprint doi: https://doi org/10.1101/2020.03.24.003590; this version posted March 25, 2020. The copyright holder for this preprint (which was not certified by peer review) is the author/funder, who has granted bioRxiv a license to display the preprint in perpetuity. It is made available under aCC-BY-ND 4.0 International license.

\section{Supplementary figures and tables}
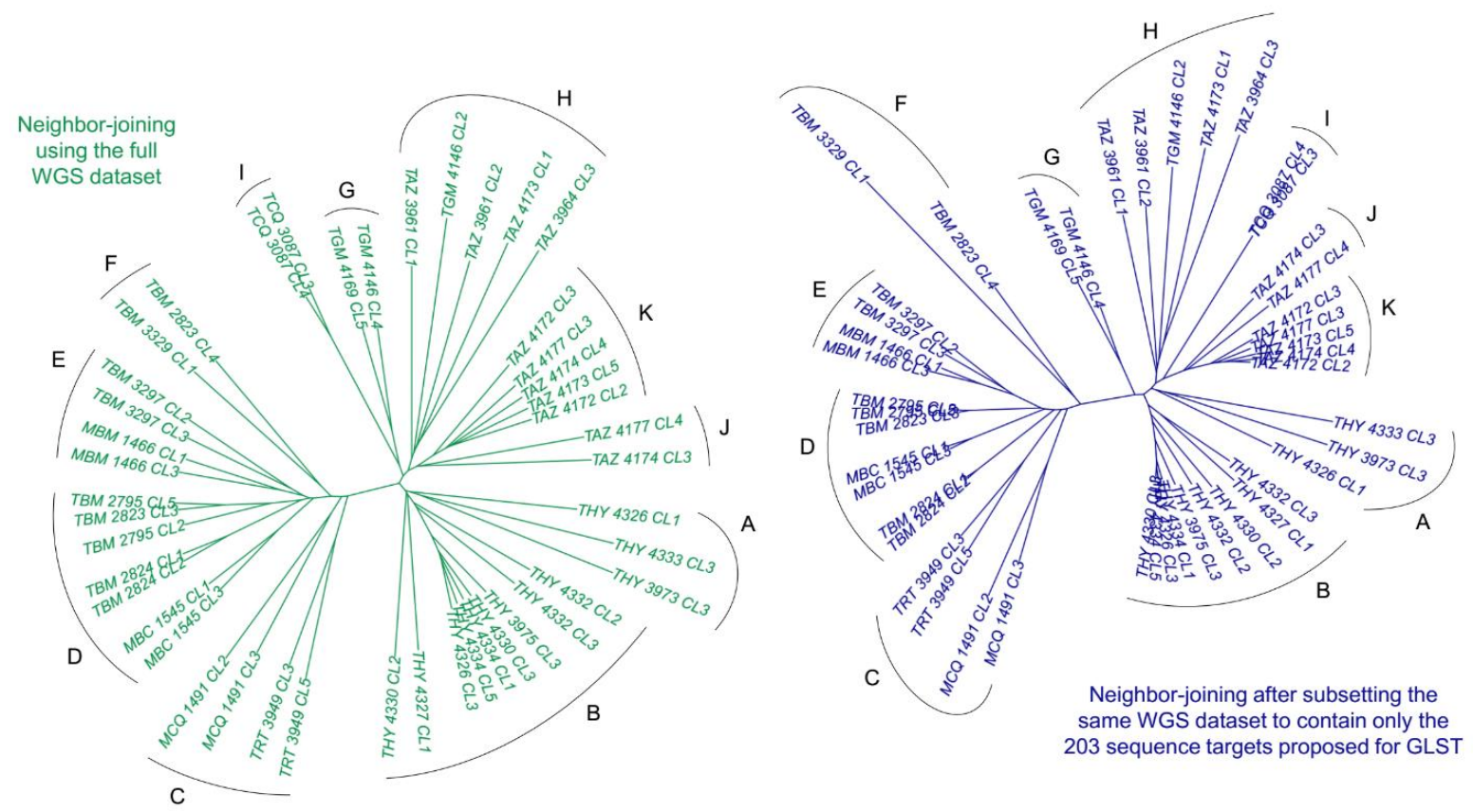

Supplementary Figure 1 Phylogenetic resolution at GLST loci in silico. The green tree shows neighbor-joining (NJ) relationships calculated from 106,007 SNP sites identified from whole-genome sequencing (WGS) of 45 Tcl clones in southern Ecuador ${ }^{1}$. Sites missing genotypes in $\geq 10 \%$ individuals are excluded. Less than $45 \mathrm{~km}$ separate the most distant sampling sites within the study region. Several pairs of clones also represent the same host/vector individual (see first seven characters of IDs). NJ was repeated after abridging the WGS dataset to contain only SNPs within the 203 sequence targets proposed by GLST (also excluding sites missing $\geq 10 \%$ genotypes). This resultant tree (blue, at right) uses 391 SNP sites and recreates clusters A-K observed in WGS.

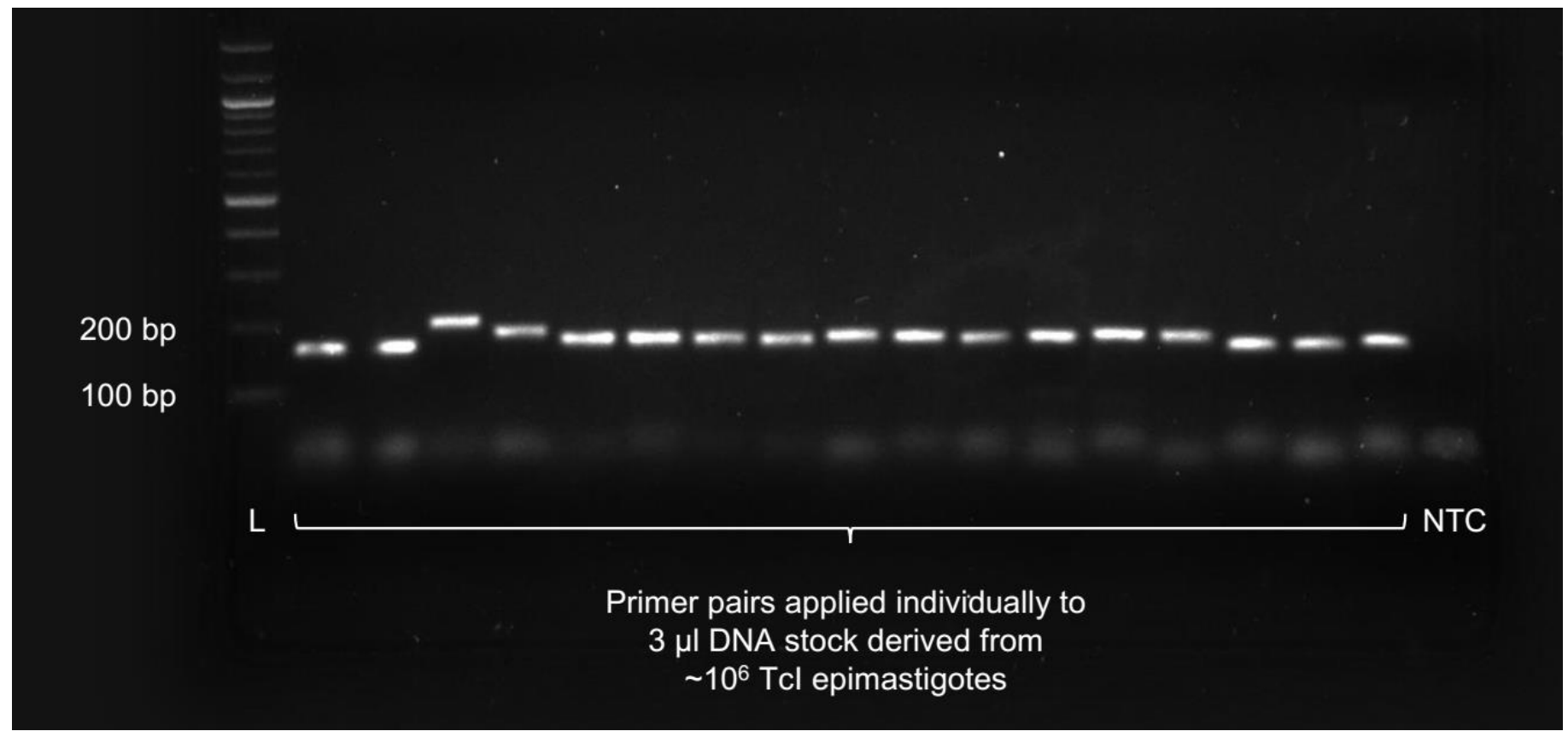

Supplementary Figure 2 Individual primer pair validation. Primer pairs were first applied individually to pure Tcl epimastigote DNA to confirm product amplification within the expected size range (164-204 bp). The figure shows the electrophoresed products of 17 different primer pairs in $0.8 \%$ agarose gel as well as DNA ladder (L) and no-template control (NTC). All other primer pairs achieved similar results using an initial incubation step at $98{ }^{\circ} \mathrm{C}(2 \mathrm{~min}) ; 30$ amplification cycles at $98^{\circ} \mathrm{C}(10 \mathrm{~s}), 60^{\circ} \mathrm{C}(30 \mathrm{~s})$, and $72{ }^{\circ} \mathrm{C}(45 \mathrm{~s})$; and a final extension step at $72{ }^{\circ} \mathrm{C}(2 \mathrm{~min})$. 


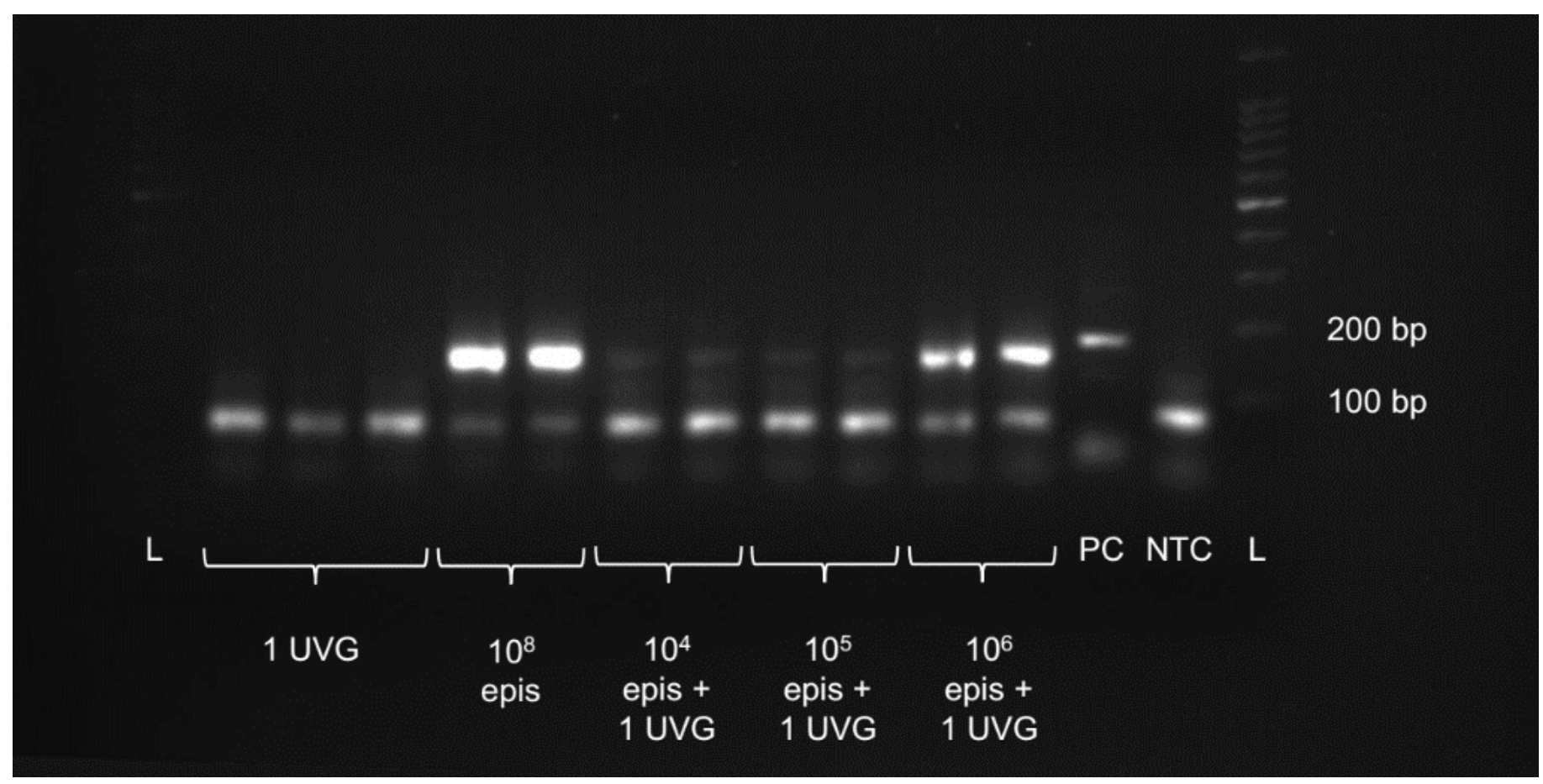

Supplementary Figure 3 Preliminary GLST (multiplex) trials on T. cruzi I mock infections. We created mock infections by mixing $10^{4}, 10^{5}$ and $10^{6}$ RNAlater-preserved Tcl-Sylvio epimastigote (epi) cells with uninfected Rhodnius prolixus vector gut (UVG). DNA extracted from these mock infections was subjected to the multiplexed, 203-target GLST reaction (using the same cycling conditions as for single-target reactions - see Methods or Supplementary Fig. 2 legend) and products were electrophoresed in $0.8 \%$ agarose gel. Fainter banding of GLST products from lower concentration mock infections encouraged follow-up on sensitivity thresholds using additional dilution curves and qPCR. Next to DNA ladder (L) and no-template control (NTC), the gel also contains TcZ primer product from pure Tcl epimastigote DNA. TcZ primers provide a highly sensitive positive control (PC) as they target 195 bp satellite DNA repeats that make up ca. $5 \%$ of the T. cruzi genome.

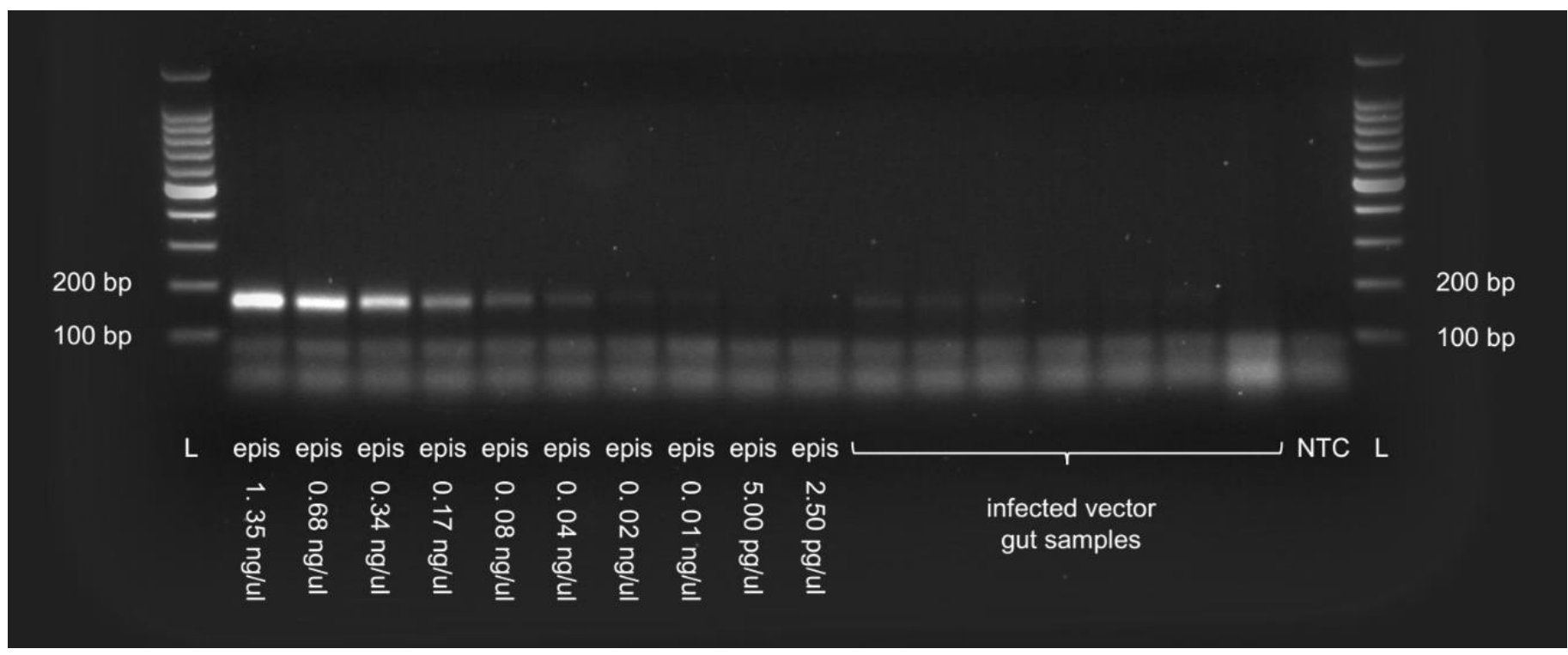

Supplementary Figure 4 T. cruzi I DNA dilutions and GLST product visibility in $0.8 \%$ agarose gel. The left side shows electrophoresed GLST amplicons generated from $3 \mu \mathrm{l}$ pure Tcl epimastigote (epi) DNA with concentrations between $1.35 \mathrm{ng} / \mu \mathrm{l}$ and $2.50 \mathrm{pg} / \mu \mathrm{l}$ (see cycling conditions in Methods or Supplementary Fig. 2 legend). Lanes on the right contain amplicons from seven random metagenomic samples that tested positive for $T$. cruzi satellite DNA (not shown). DNA ladders (L) and no-template control (NTC) are indicated left and right. Poor amplicon visibility occurs at $\leq 60 \mathrm{pg}$ epimastigote DNA input. Gut DNA amplicon visibility is also limited but whether this relates to low T. cruzi content or amplification interference is unclear without QPCR. 
bioRxiv preprint doi: https://doi.org/10.1101/2020.03.24.003590; this version posted March 25, 2020. The copyright holder for this preprint (which was not certified by peer review) is the author/funder, who has granted bioRxiv a license to display the preprint in perpetuity. It is made available under aCC-BY-ND 4.0 International license.

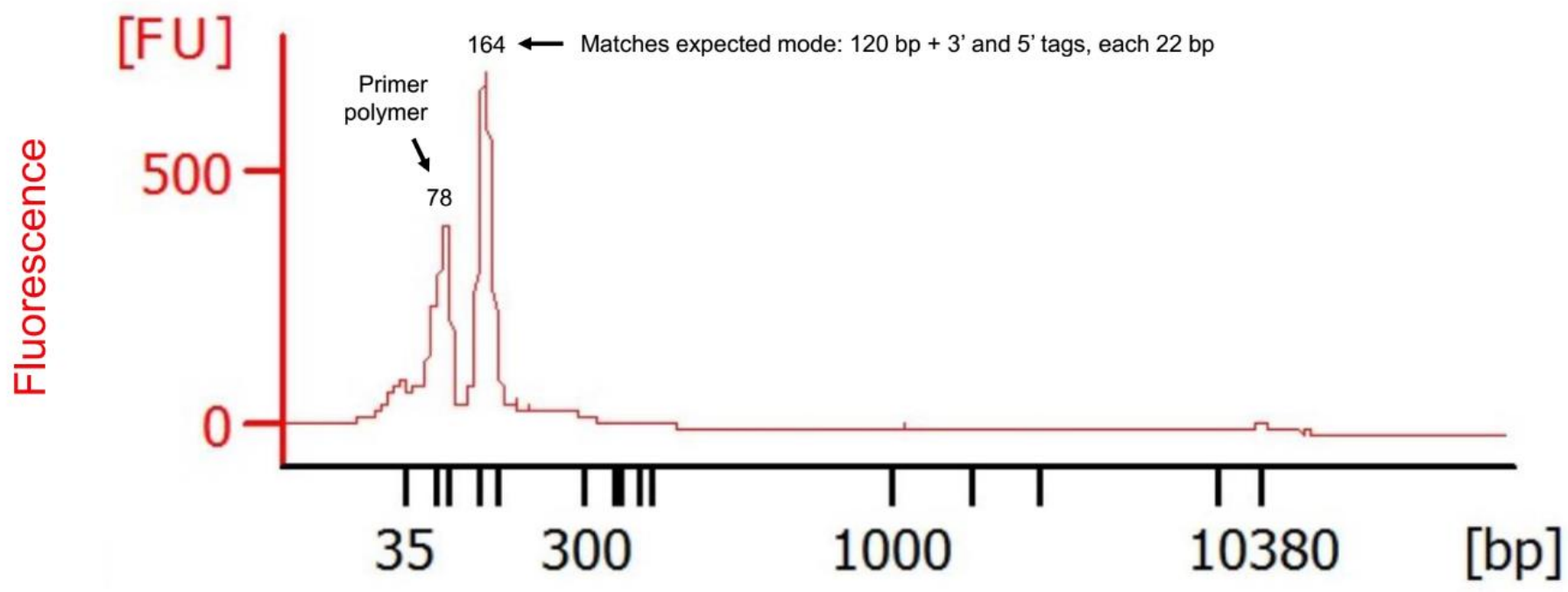

\section{Fragment length}

Supplementary Figure 5 First-round (unbarcoded) PCR product size composition measurement using microfluidic electrophoresis. The figure plots fragment sizes (calculated based on migration times relative to those of standards) and fluorescence intensity (FU) of first-round PCR products (see cycling conditions in Methods or Supplementary Fig. 2 legend) measured with the Agilent Bioanalyzer 2100 System. The first peak represents primer polymerization that is removed in subsequent gel excision/re-solubilization steps. The second peak matches expectations for the multi-target GLST product (164 - 204 bp). Special thanks to Craig Lapsley at the Wellcome Centre for Molecular Parasitology in Glasgow for generating this data.
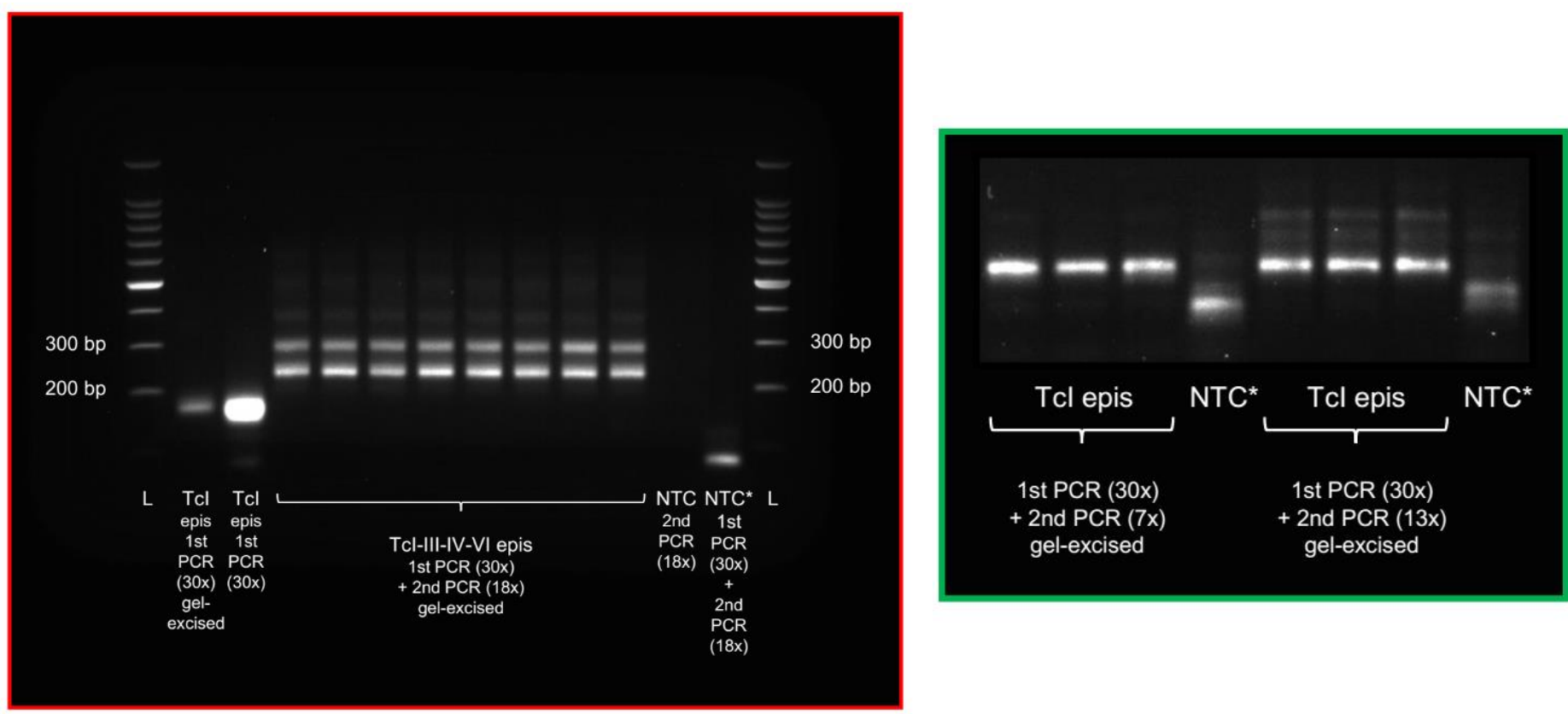

Supplementary Figure 6 Large polymer formation from excessive amplicon barcoding. The second (barcoding) PCR reaction uses an initial incubation step at $98^{\circ} \mathrm{C}(2 \mathrm{~min}) ; 7$ amplification cycles at $98{ }^{\circ} \mathrm{C}(30 \mathrm{~s}), 60^{\circ} \mathrm{C}(30 \mathrm{~s})$, and $72{ }^{\circ} \mathrm{C}$ ( $1 \mathrm{~min}$ ); and a final extension step at $72^{\circ} \mathrm{C}(3 \mathrm{~min})$. Seven amplification cycles were chosen because unwanted polymers formed at 13 and $18 x$. The center lanes in the $0.8 \%$ agarose gel at left (red border) show electrophoresed GLST products from reference clones after eighteen cycles of barcoding PCR. Large, non-target banding occurs at $\geq 300 \mathrm{bp}$. Unbarcoded products from Tcl epimastigote (epi) DNA are also shown at left. No template controls from barcoding (NTC) and first-round + barcoding PCR (NTC*) occur next to the DNA ladder (L) on the right side of the gel. The smaller image (green border) to the right shows how unwanted banding becomes less pronounced at $13 x$ and largely disappears at $7 \mathrm{x}$. This $0.8 \%$ agarose gel also contains NTC* samples, i.e., negative controls carried through both first and secondround PCR. 
bioRxiv preprint doi: https://doi.org/10.1101/2020.03.24.003590; this version posted March 25, 2020. The copyright holder for this preprint (which was not certified by peer review) is the author/funder, who has granted bioRxiv a license to display the preprint in perpetuity. It is made available under aCC-BY-ND 4.0 International license.

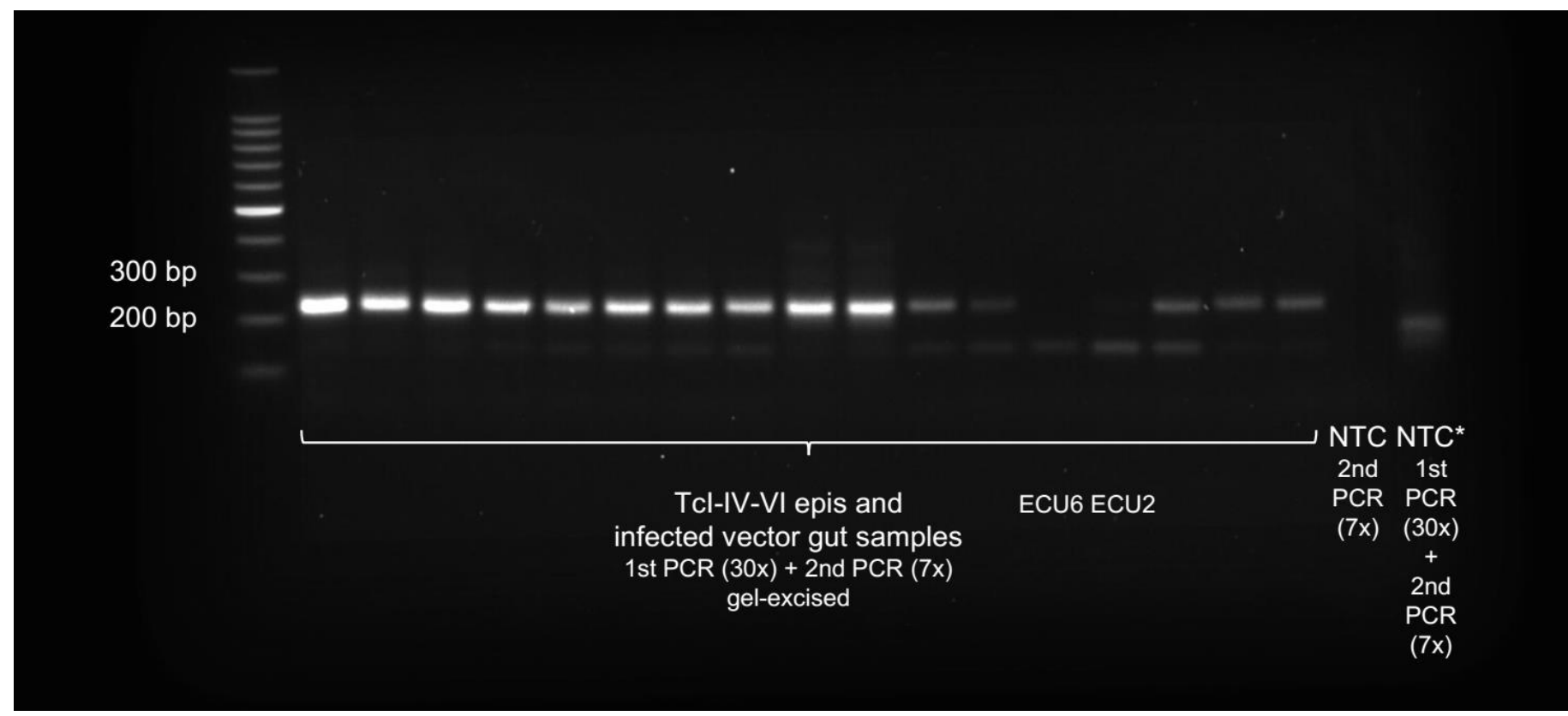

Supplementary Figure 7 Barcoded GLST products ready for final pooling and purification. The $0.8 \%$ agarose gel shows a subset of fifteen GLST products from the second-round (barcoding) PCR reaction (see cycling conditions in Methods or Supplementary Fig. 6 legend) prior to equimolar pooling and final gel excision/re-solubilization steps. Products from ECU6 and ECU2 occur in this gel but were not included in the final pool. The gel also contains DNA ladder $(L)$ and notemplate controls from barcoding (NTC) and first-round + barcoding PCR (NTC*).

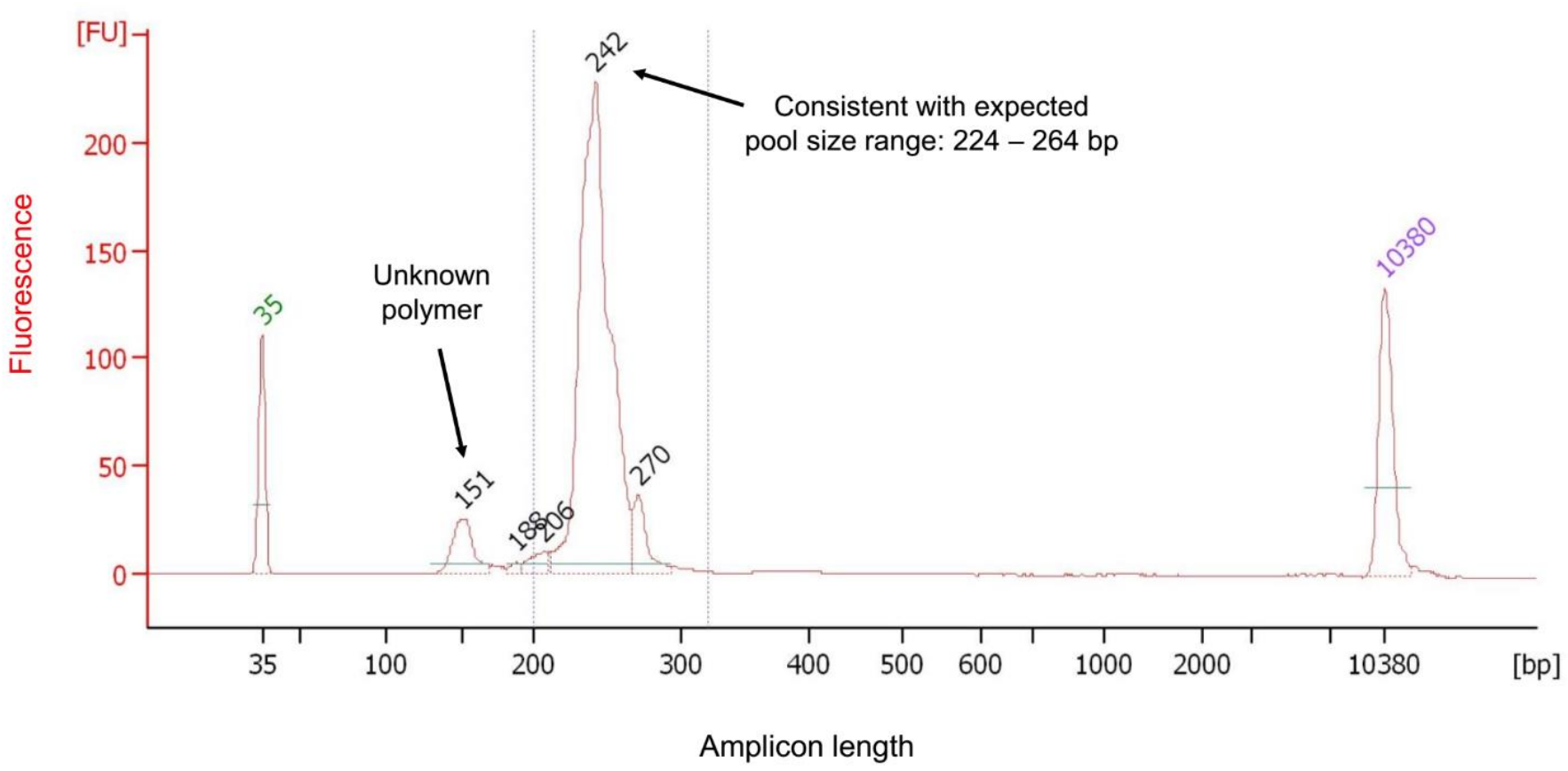

Supplementary Figure 8 Final (barcoded) GLST pool size composition measurement using microfluidic electrophoresis. The figure plots fragment sizes (calculated based on migration times relative to those of standards) and fluorescence intensity (FU) of the final GLST pool measured with the Agilent Bioanalyzer 2100 System. The large peak matches expectations for the multi-target GLST product pool (224-264 bp). Left and right peaks labelled in green and purple represent standards of known size. A small non-target peak remaining near 151 bp encourages improvement of prior size selection steps. Special thanks to Julie Galbraith at Glasgow Polyomics for generating this data. 


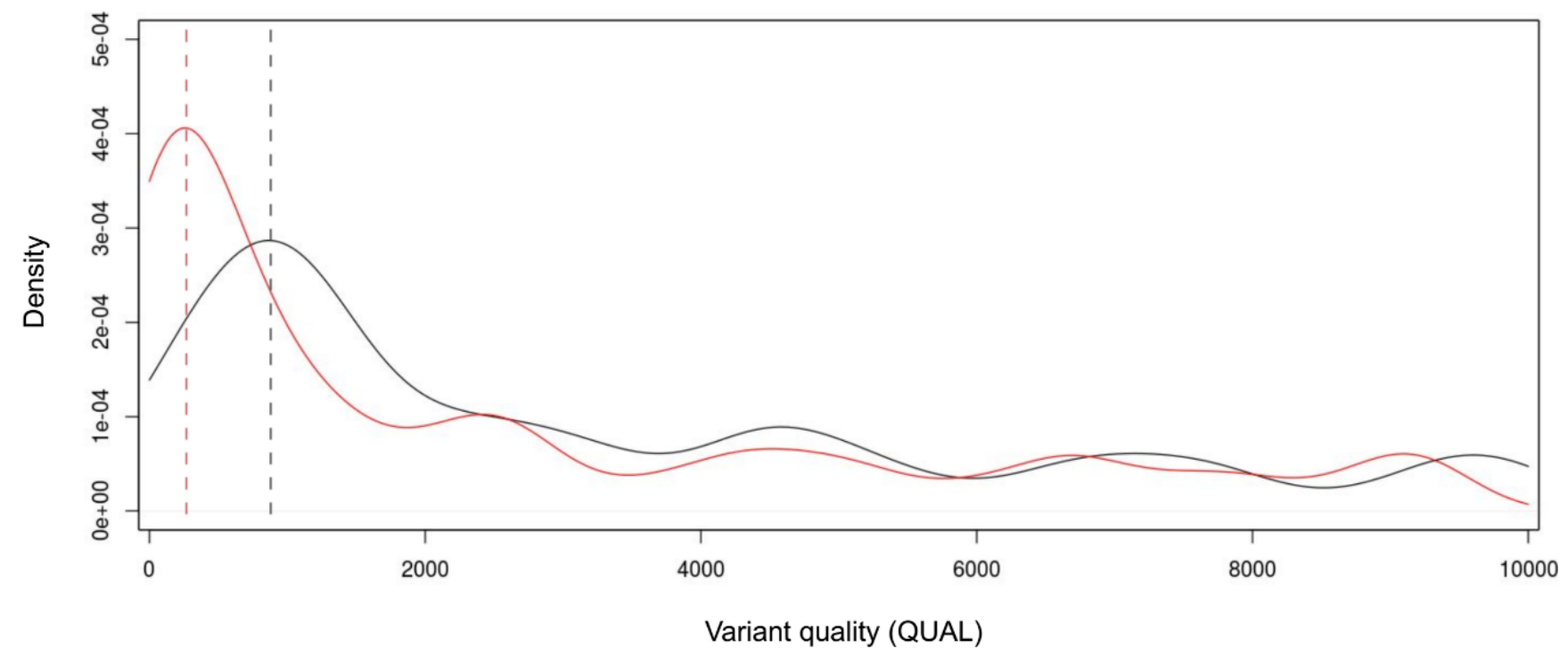

Supplementary Figure 9 Quality scores at previously identified vs. unidentified variant sites. The GLST primer panel was designed based on single-nucleotide polymorphisms (SNPs) in Ecuadorian Tcl clones. It was applied, however, to samples from distant geographic locations as well as to non-Tcl clones. Additional, previously unidentified SNP sites (PU) were thus expected to be found but we needed to distinguish true PU from PCR and sequencing error. We reasoned that quality statistics (e.g., mapping quality, strand bias, minor allele frequency, etc. - see Methods) at previously identified SNP sites (PI) could help calibrate quality filters applied to the wider dataset. This strategy finds support in the above density plot of QUAL scores computed by Genome Analysis Toolkit 42 . The plot suggests that, prior to variant filtration, lower QUAL scores occur more often at PU (red) than at PI (black). We thus imposed the most stringent filtering criteria possible without losing PI.

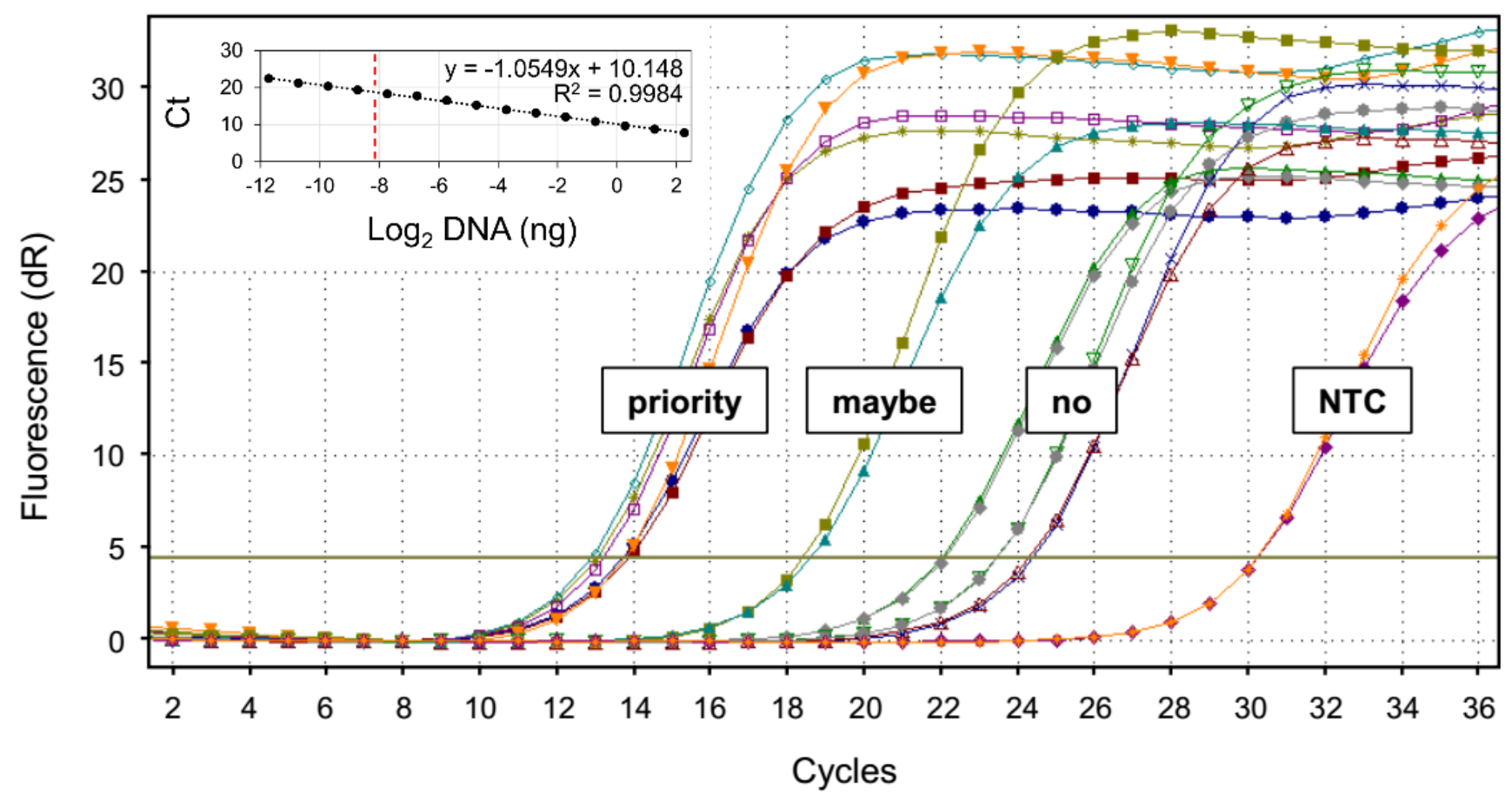

Supplementary Figure 10 GLST sample selection and sensitivity estimation via qPCR. We used T. cruzi satellite DNA qPCR to identify vector gut samples with $T$. cruzi DNA quantities within ranges successfully visualized in GLST reactions using epimastigote DNA (Supplementary Fig. 4). The qPCR reaction used an initial incubation step at $95{ }^{\circ} \mathrm{C}(10 \mathrm{~min})$ and 40 amplification cycles at $95{ }^{\circ} \mathrm{C}(15 \mathrm{~s}), 55^{\circ} \mathrm{C}(15 \mathrm{~s})$, and $72{ }^{\circ} \mathrm{C}(15 \mathrm{~s})$. The plot shows baseline-corrected fluorescence $(\mathrm{dR})$ for seven sample duplicates. Following the regression equation from the standard curve (see inset), the three samples with highest cycle thresholds (Ct values) in this example represent gut extracts with 0.05 to $0.14 \mathrm{ng} / \mu \mathrm{l}$ T. cruzi DNA. Such samples with T. cruzi DNA concentrations above $0.01 \mathrm{ng} / \mu \mathrm{l}$ were prioritized for GLST and none failed in library construction. ECU36, with a mean Ct value of 18.68 in the plot, was also successfully sequenced. A Ct value of 18.68 represents $3.69 \mathrm{pg} / \mu \mathrm{l} T$. cruzi DNA. Not all samples with concentrations at single-digit picogram levels $($ per $\mu l)$ were successful and we did not troubleshoot those with substantially lower concentrations based on qPCR. 


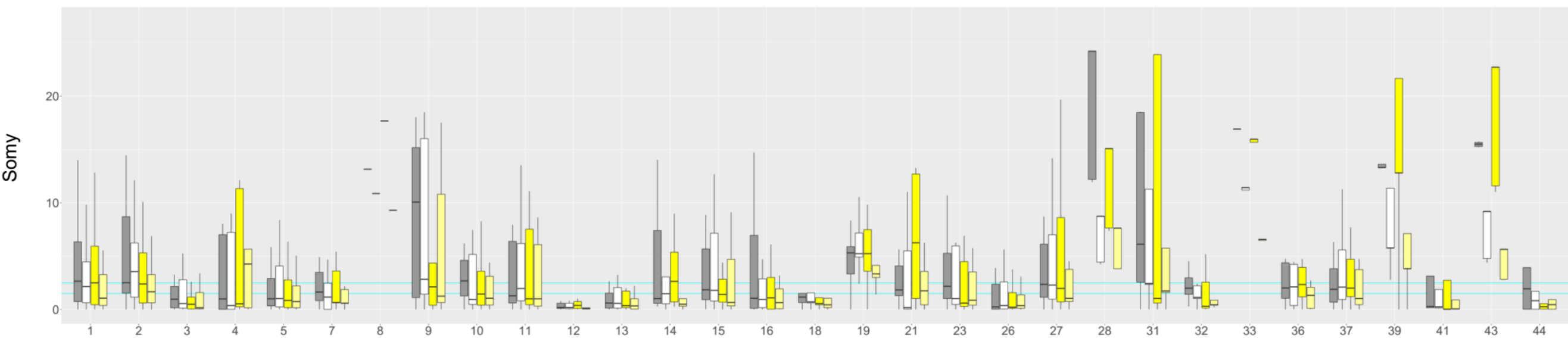

Chromosome

Supplementary Figure 11 Target coverage in control replicates confirms expectations that the GLST panel applied in this study is unreliable for copy number estimation. We adapted methods from Schwabl et al. $2019^{1}$ to derive somy estimates for each base position within GLST amplicons. Briefly, we calculated median-read-depth of all target bases for each chromosome. We let the median of these chromosomal medians (the 'inter-chromosomal median') represent expectations for the disomic state, estimating copy number per base position by dividing each position's read-depth by the inter-chromosomal median and multiplying by two. Boxplots show median and interquartile ranges of these site-wise somy estimates for each chromosome in TBM 2975 CL2 control replicates. TBM 2795 CL2 did not show chromosomal amplifications in whole-genome analysis ${ }^{1}$. Not unexpectedly for a PCR-based method, somy values estimated from GLST read-depths differ substantially among replicates and are unrealistically high/low on many chromosomes. Estimates on chromosomes with few GLST targets appear especially unreliable - e.g., see chromosomes 8, 28, 33, 39 and 43. These chromosomes contain $\leq 2$ GLST targets each. The horizontal lines cyan lines mark $y=1.5$ and $y=2.5$. 


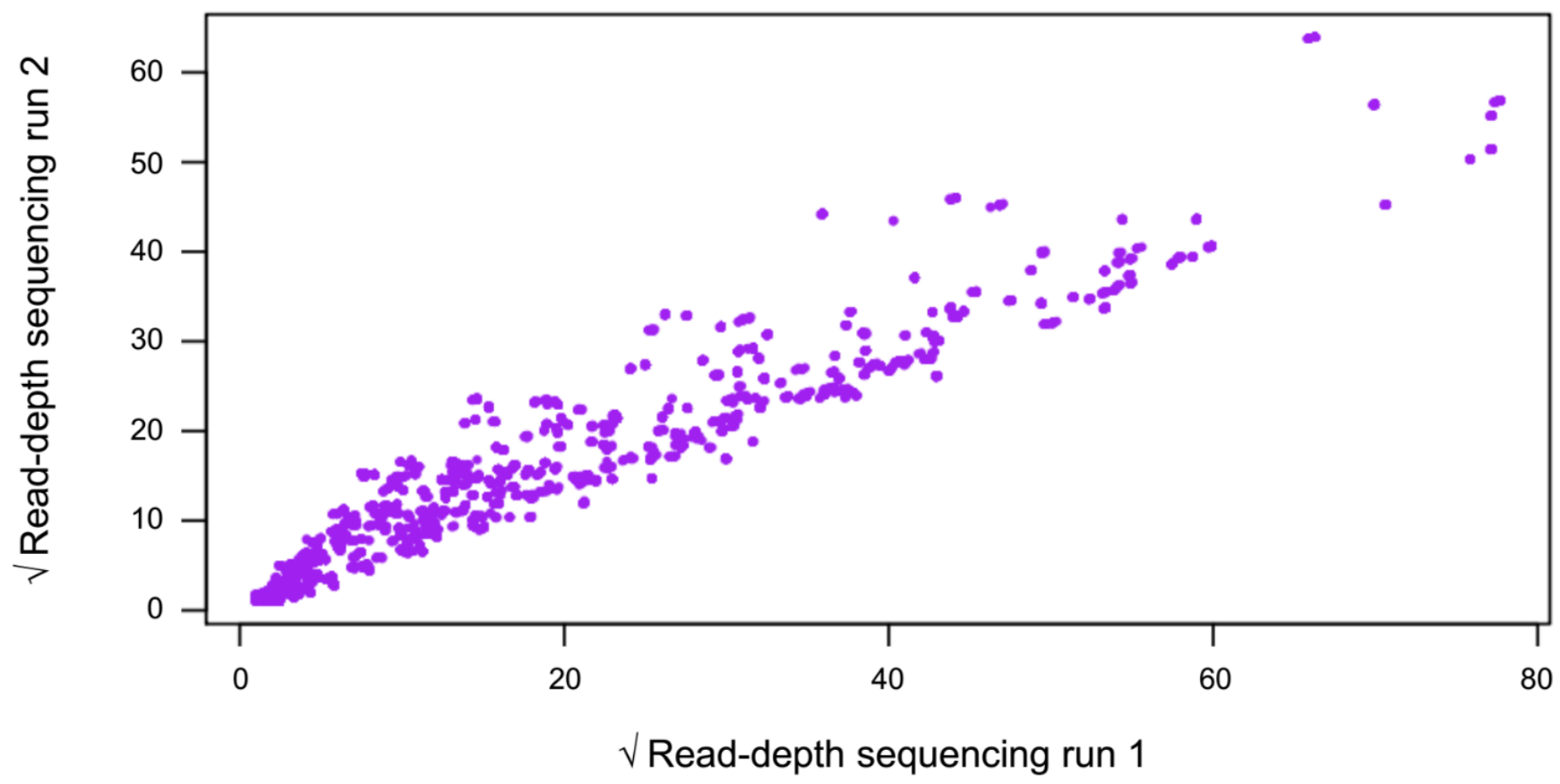

Supplementary Figure 12 Similar read-depth distribution between separate sequencing runs. We sequenced the same GLST pool in two separate Illumina MiSeq runs. Run 1 involved GLST as a spike to a collaborator's $16 S$ amplicon library, whereby GLST reads were subsequently decontaminated from (barcode-sharing) $16 \mathrm{~S}$ reads by alignment to the Tcl-Sylvio reference genome. Run 2 was dedicated solely to GLST, i.e., no non-GLST libraries were simultaneously sequenced on the flow cell. The plot shows that run 1 and run 2 read-depths at each GLST base position (purple points) are highly correlated (Pearson's $r=0.93, p<0.001$ ), and that run 1 had higher sequencing output than run 2 . Readdepth values are square-root transformed and represent control sample TBM_2975_CL2_rep1. 
Supplementary Table 1 Details on T. cruzi-infected metagenomic triatomine gut samples from Colombia (COL), Venezuela (VZ) and Ecuador (ECU). Abbreviations: Dep. (Department); Met. Caracas (Metropolitan District of Caracas); EPSG (European Petroleum Survey Group coordinate system); reps. (technical replicates).

ID

COL77

COL78

COL133

COL135

COL154

COL155

COL169

COL253

COL319

COL466

COL468

ECU3

ECU4

ECU8

ECU9

ECU10

ECU36

ECU41

ECU77

TBM_2795_CL2

VZ13516 Panstrongylus geniculatus

VZ35814 Panstrongylus geniculatus

VZ6616 Panstrongylus geniculatus

VZ1214D Panstrongylus geniculatus

VZ16816 Panstrongylus geniculatus

VZ17114 Panstrongylus geniculatus

\section{Region}

Santander Dep.

Santander Dep.

Casanare Dep.

Casanare Dep.

Casanare Dep.

Casanare Dep.

Casanare Dep.

Casanare Dep.

Arauca Dep.

Boyacá Dep.

Boyacá Dep.

Loja Province

Loja Province

Loja Province

Loja Province

Loja Province

Loja Province

Loja Province

Loja Province

Loja Province

Met. Caracas

Met. Caracas

Met. Caracas

Met. Caracas

Met. Caracas

Met. Caracas

Met. Caracas
Municipality / community

Lebrija

Lebrija

Paz de Ariporo

Paz de Ariporo

Tamara

Tamara

Pore

Paz de Ariporo

Fortul

Soata

Soata

Bramaderos

Bramaderos

Bramaderos

Bramaderos

Bramaderos

Galápagos

Guineo

Jacapo

Bella Maria

Libertador

Libertador

Libertador

Sucre

Sucre

Sucre

Sucre

X (EPSG 3786)
-8141577.9370
-8141577.9370
-7993997.4220
-7993997.4220
-8024081.7980
-8024081.7980
-8005271.3760
-7993997.4220
-7980623.1040
-8083880.0490
-8083880.0490
-8875849.2150
-8875849.2150
-8875849.2150
-8875849.2150
-8875849.2150
-8832711.9860
-8899431.9060
-8830688.2360
-8852271.1950
-7447967.9080
-7441110.8420
-7450655.1580
-7426686.3980
-7427396.8230
-7427026.2100
-7426501.1470

x (EPSG 3786)

y (EPSG 3786)

790936.6092

790936.6092

653950.4247

653950.4247

648298.0468

648298.0468

636869.6421

653950.4247

755354.1935

704231.6027

704231.6027

$-453603.4112$

$-453603.4112$

$-453603.4112$

$-453603.4112$

$-453603.4112$

$-483957.8804$

$-466731.6546$

$-485500.9341$

$-466705.6350$

1167084.6630

1169154.1140

1165756.5490

1163934.1740

1166961.1250

1162328.0720

1162853.1350

\section{Ecotope}

Sylvatic

Sylvatic

Domestic

Domestic

Domestic

Domestic

Domestic

Domestic

Domestic

Unknown

Unknown

Sylvatic

Sylvatic

Sylvatic

Sylvatic

Sylvatic

Sylvatic

Sylvatic

Sylvatic

Domestic

Domestic

Domestic

Domestic

Domestic

Domestic

Domestic

Domestic

$\begin{array}{cc}\text { Year } & \text { Reps } \\ 2015 & 1 \\ 2015 & 2 \\ 2016 & 2 \\ 2016 & 2 \\ 2016 & 2 \\ 2016 & 2 \\ 2016 & 1 \\ 2016 & 1 \\ 2016 & 2 \\ 2017 & 3 \\ 2017 & 3 \\ 2009 & 2 \\ 2009 & 2 \\ 2009 & 1 \\ 2009 & 2 \\ 2009 & 2 \\ 2009 & 1 \\ 2009 & 1 \\ 2008 & 1 \\ 2009 & 4 \\ 2016 & 2 \\ 2016 & 2 \\ 2014 & 2 \\ 2016 & 2 \\ 2014 & 1 \\ 2016 & 1 \\ 2014 & 1\end{array}$


Supplementary Table 2 GLST primer sequences. The 3' end of each first-round PCR primer is target-specific. The 5' end of each forward primer contains CS1. The 5' end of each reverse primer contains CS2. These sequencing primer binding sites are shown in pink. In subsequent barcoding PCR, the reverse primer consists of

5'-CAAGCAGAAGACGGCATACGAGAT ${ }^{*}{ }^{*}$ TACGGTAGCAGAGACTTGGTCT-3', where * $X^{*}$ is a unique 10 nt barcode used to label each sample's sequence reads. The reverse barcoding primer also contains CS2. The forward barcoding primer (5'-AATGATACGGCGACCACCGAGATCTACACTGACGACATGGTTCTA-3') contains CS1 and is the same for all samples.

\section{ID}

TC_LOJ_1

TC_LOJ_2

TC_LOJ_4

TC_LOJ_5

TC_LOJ_6

TC_LOJ_8

TC_LOJ_9

TC_LOJ_10

TC_LOJ_11

TC_LOJ_12

TC_LOJ_13

TC_LOJ_14

TC LOJ 15

TC_LOJ_16

TC_LOJ_19

TC_LOJ_20

TC_LOJ_23

TC_LOJ24

TC LOJ 25

TC_LOJ_26

TC_LOJ_27

TC_LOJ_28

TC_LOJ_29

TC LOJ 30

TC LOJ 32

TC_LOJ_34

\section{Target region}

chr16:130780-130919

chr10:534441-534583

chr11:368075-368194

chr1:2082456-2082586 chr12:1011748-1011869 chr5:515822-515951 chr1:163164-163296 chr1:1104374-1104501 chr5:995176-995297 chr14:833083-833213 chr23:560603-560743 chr19:763581-763703 chr4:1431898-1432017 chr16:1168122-1168248 chr43:177414-177556 chr26:294140-294261 chr18:690694-690813 chr1:1993894-1994026 chr36:470603-470728 chr13:433737-433859 chr24:269253-269379 chr27:389665-389794 chr36:451747-451871 chr7:1140939-1141071 chr2:120852-120972 chr16:170448-170597

\section{Forward primer sequence (5'-3')}

ACACTGACGACATGGTTCTACATGCCAATAACGGTCAAAGTAAACG ACACTGACGACATGGTTCTACAAGAGTTGTGGCATCCTTGTTCTTG ACACTGACGACATGGTTCTACAAGGAGGTGAAACGGATGGTAAAGA ACACTGACGACATGGTTCTACAAGCTCAAGGGCTGAAATAGACACA ACACTGACGACATGGTTCTACACCACTCTATCGTCTACGCATCCTC ACACTGACGACATGGTTCTACAAATGGAGATGGAGGATATGAAGCA ACACTGACGACATGGTTCTACACGCTGAGTATCAATTTAAGCGTAGCA ACACTGACGACATGGTTCTACATGCCCTTCACATTTATCCCAAGTA ACACTGACGACATGGTTCTACAGCAACTCCACAAACGACTCAGAAC ACACTGACGACATGGTTCTACACTTGTTGCTAAGTGTCCGTGTGTC ACACTGACGACATGGTTCTACAGTCTTTGATTTCTCGTCCGTACCTT ACACTGACGACATGGTTCTACAAAGATACAAGAGCACGGTACAAAGGA ACACTGACGACATGGTTCTACAAGGACTATGCTCAAGACGGGATCT ACACTGACGACATGGTTCTACATACAAACATCAACGCAGAACATGC ACACTGACGACATGGTTCTACACAGTCCTCCAGTTCTCCAAGTGAT ACACTGACGACATGGTTCTACAGCACAAGAACGGGTGTACCTTCTA ACACTGACGACATGGTTCTACAAAAGAAACTTCGGGTAGCGACAAC ACACTGACGACATGGTTCTACATTCTACACACTCCGCCTTACGTCT ACACTGACGACATGGTTCTACAGTGGCTCAGAAGCATGATCGTAAT ACACTGACGACATGGTTCTACACAATGGTGATGATGAGGTTAAGCA ACACTGACGACATGGTTCTACAGGCGATAAGGAAGAATGGAGAGAA ACACTGACGACATGGTTCTACAACCACTTCACCATTTGTCTGGTATTC ACACTGACGACATGGTTCTACAGTGTGTTTGAGATTGGGCCTGTAT ACACTGACGACATGGTTCTACAAGTTGATCGTCTTTCTTCCTTGACC ACACTGACGACATGGTTCTACAAAATGATGTACTGCCTGAACTGGAA ACACTGACGACATGGTTCTACAGGAAGAAGGCAGACTAAACAGGATG

\section{Reverse primer sequence (5'-3')}

TACGGTAGCAGAGACTTGGTCTGCACACGAAGGTACACTCACTTCC TACGGTAGCAGAGACTTGGTCTAAACGCCTTCACCTTACTCAGACA TACGGTAGCAGAGACTTGGTCTTGCGAAGAAGAAGATCAAACTCTCTC TACGGTAGCAGAGACTTGGTCTCGTTTAGGCTGGAAAGATGGAAGT TACGGTAGCAGAGACTTGGTCTATCATCTTGAGACACATGCCTTGC TACGGTAGCAGAGACTTGGTCTTTTAGACCTCATGTTTCCCGTGTC TACGGTAGCAGAGACTTGGTCTACCCATATCCGTCATCCCTATTGT TACGGTAGCAGAGACTTGGTCTAAATAGCATGGAACTCAGCCAGAA TACGGTAGCAGAGACTTGGTCTGATGCTGCCATTTCGTCTTTACTC TACGGTAGCAGAGACTTGGTCTGCCTTTATATTGATCGGCTCCTCT TACGGTAGCAGAGACTTGGTCTTGCATCTTCTACTTTCTCGGAAGC TACGGTAGCAGAGACTTGGTCTGTGAAGAGGGATGGATCAACATTC TACGGTAGCAGAGACTTGGTCTCATCAAGTGGACACAACAGCAACT TACGGTAGCAGAGACTTGGTCTCACACATCCCGTAACTCAATGGTA TACGGTAGCAGAGACTTGGTCTGAGATTGTTCTCTCTGTCCCAACG TACGGTAGCAGAGACTTGGTCTTGTGTCGAGGGAATTGATTACTGC TACGGTAGCAGAGACTTGGTCTCACCACTTCTGCTAGACCACATCC TACGGTAGCAGAGACTTGGTCTGTCTGCAACGACACATAGATTGGA TACGGTAGCAGAGACTTGGTCTACCCTTGTAGTCTTCGCAGTCCTC TACGGTAGCAGAGACTTGGTCTACGTCCAATACACACAAACACACAG TACGGTAGCAGAGACTTGGTCTGTCATGTGCTTACGAGAGCCGTAG TACGGTAGCAGAGACTTGGTCTTTTAAGATGGCCGCATACAGTGAG TACGGTAGCAGAGACTTGGTCTCACATCAAGTACCTCCGTGTACGA TACGGTAGCAGAGACTTGGTCTAAATGTTCCTGCGTACACCAAGTC TACGGTAGCAGAGACTTGGTCTGTTCTCCGCCGTATTCTCCTCTAC TACGGTAGCAGAGACTTGGTCTAGCTTGTCACTGCTCACAGAGTTG 
TC_LOJ_35 chr26:125032-125153 TC_LOJ_36 chr5:1012765-1012911 TC_LOJ_37 chr1:2889409-2889535 TC_LOJ 38 chr21:465093-465213 TC_LOJ_39 chr1:1160205-1160334 TC_LOJ_40 chr7:1138368-1138496 TC_LOJ_41 chr1:2693345-2693466 TC_LOJ_42 chr10:1016129-1016269 TC_LOJ 43 chr1:1956698-1956821 TC_LOJ_44 chr3:173883-174019 TC_LOJ_45 chr3:174152-174277 TC_LOJ_46 chr1:1833807-1833948 TC_LOJ_47 chr14:844524-844671 TC_LOJ_48 chr3:1058072-1058196 TC_LOJ51 chr12:596775-596914 TC_LOJ_52 chr31:428464-428593 TC_LOJ_54 chr2:925727-925855 TC_LOJ_55 chr12:306151-306272 TC_LOJ_56 chr21:341510-341636 TC_LOJ_57 chr37:454539-454662 TC_LOJ_58 chr15:395493-395614 TC_LOJ_59 chr2:856618-856737 TC_LOJ_60 chr26:139346-139478 TC_LOJ_61 chr1:1992854-1992995 TC_LOJ_62 chr1:305886-306012 TC LOJ 63 chr26:303994-304113 TC_LOJ_64 chr14:889253-889389 TC_LOJ_67 chr10:143080-143202 TC_LOJ_69 chr2:446791-446914 TC_LOJ 70 chr32:839405-839556 TC_LOJ_71 chr7:179338-179460
ACACTGACGACATGGTTCTACAGTACGCTACACTGCGAGAGGAATG ACACTGACGACATGGTTCTACATCCGTCCCTGTTGTCTTCTCAATA ACACTGACGACATGGTTCTACACAGAGTTCCACGGATAAGTCGTCA ACACTGACGACATGGTTCTACATGGTTGTAGTCCGTGATCTCTGGT ACACTGACGACATGGTTCTACAACGTCACATTTGTACTGCGAGAGG ACACTGACGACATGGTTCTACAGTCCAAGCCGTTGTCTCTCAATAC ACACTGACGACATGGTTCTACATGGCTGGTGCAAATGTACTCATATC ACACTGACGACATGGTTCTACATACGACTCCCTTTCCACATACGAC ACACTGACGACATGGTTCTACAGCTCTCATGGGTGGTAGAAGCTAA ACACTGACGACATGGTTCTACAGTCATCATTCTCGGAAACAAAGTAGG ACACTGACGACATGGTTCTACAAGTACGCCACACGACAGTTCAGTT ACACTGACGACATGGTTCTACAATTCGTGTCATTAGCAGCAGCAAC ACACTGACGACATGGTTCTACAAGCAATTCACGGAGTTCACAGATG ACACTGACGACATGGTTCTACAGATAGCACAAACAAGCCAAATGGT ACACTGACGACATGGTTCTACAGATTGACATTACGGCGATTCAGAG ACACTGACGACATGGTTCTACACCCTCATGGAGACATCTACGAATCT ACACTGACGACATGGTTCTACAAATGCTAGAGGGCGATAATGAAGAC ACACTGACGACATGGTTCTACATGGGTCTGCTTGACTGGTTTCTTA ACACTGACGACATGGTTCTACAATACTCCTCTGCATTCACCTCCTG ACACTGACGACATGGTTCTACAGTACGTGAAACGCCCTGACTTTAC ACACTGACGACATGGTTCTACACTTTGTGACCACCTCCTTGTTATTG ACACTGACGACATGGTTCTACAGCCCGGTTCACAACTTTAGTAGAAA ACACTGACGACATGGTTCTACAGATTATGGTGGTGGTTTCAACACG ACACTGACGACATGGTTCTACAATCTGTTGAGGATGACCGAACACT ACACTGACGACATGGTTCTACATACTCAGGCGTAGAAACAGGCTCA ACACTGACGACATGGTTCTACACATGACAAGCATAAATACAGCGAGAG ACACTGACGACATGGTTCTACACTTCCCAGACTCATCTTTCTGCTG ACACTGACGACATGGTTCTACACACTAACTGGGTCAAAGTGTTCTTGC ACACTGACGACATGGTTCTACAGGTAGAAGGTACTCTCATCGGTAGCA ACACTGACGACATGGTTCTACAGGTGCGTACTGTCTTGGAAGGTTT ACACTGACGACATGGTTCTACAATGGGAGATCGGGAGTACATGAAG
TACGGTAGCAGAGACTTGGTCTGCACAACTGAGATTATAGCCAACTCC TACGGTAGCAGAGACTTGGTCTTGAGCAAAGTGTCCTTATTCTTCAGC TACGGTAGCAGAGACTTGGTCTACACACTTCCAGATCACTACGAAGC TACGGTAGCAGAGACTTGGTCTATAACTGGTTCGGGAAGGAAGAAA TACGGTAGCAGAGACTTGGTCTCCCTTACTTGTCTCCGACTCATTCT TACGGTAGCAGAGACTTGGTCTTGTTCGTTGTGGTGGAATGTGTAG TACGGTAGCAGAGACTTGGTCTTAAACAAGTGTGCCATTGCGTATC TACGGTAGCAGAGACTTGGTCTATATTGAGCCGAAACACGAAGTACA TACGGTAGCAGAGACTTGGTCTCCCACTGTCATTATTCAAACTGCTC TACGGTAGCAGAGACTTGGTCTGTGTCCATCAGCTCTACAATGCAC TACGGTAGCAGAGACTTGGTCTTGAGTAGTTGTGCCCTTCGATGTA TACGGTAGCAGAGACTTGGTCTGACGGTAAATTCTGCGTACACTGC TACGGTAGCAGAGACTTGGTCTAGGAGTCACCACAGAAGTCAGAGC TACGGTAGCAGAGACTTGGTCTGAAAGATACGCCTTCCAATCATCA TACGGTAGCAGAGACTTGGTCTTGTGGATCTTCTGCCATGATATTG TACGGTAGCAGAGACTTGGTCTTGAAGAACGAGTGTGCAGGTCATA TACGGTAGCAGAGACTTGGTCTACCTTTGCCTTGTGTTTACTGCTG TACGGTAGCAGAGACTTGGTCTGTACGGCGACTCACTTCCAAATAC TACGGTAGCAGAGACTTGGTCTGGTTGGTATAACCGAAGGAAATATGG TACGGTAGCAGAGACTTGGTCTTGGATGAACCTCCTTGTAGATGTTG TACGGTAGCAGAGACTTGGTCTAGGTATTTGGCATGTTTGATCTGC TACGGTAGCAGAGACTTGGTCTCACCAACACAGCTACGACAACAAC TACGGTAGCAGAGACTTGGTCTAAAGTGAATGGCAAATCCTAAGACG TACGGTAGCAGAGACTTGGTCTGAGAAATATCGCCGCACCTTCTAC TACGGTAGCAGAGACTTGGTCTTACCTCCGCTTATCAATGTTGTCC TACGGTAGCAGAGACTTGGTCTGAAGGTACAAGCAAGGAGCCATCT TACGGTAGCAGAGACTTGGTCTATTCCCGACTACTTTGGCATGATT TACGGTAGCAGAGACTTGGTCTAGCAACTGCGGATACTTGGTCTTC TACGGTAGCAGAGACTTGGTCTCAGAAACAGCTCGCCAGAAATAAA TACGGTAGCAGAGACTTGGTCTGTTGACGATCCACGGAAAGATATG TACGGTAGCAGAGACTTGGTCTTGAAGAGCCAAATGGGACACTAAT 
Supplementary Table 2 (continued)

TC_LOJ_74

TC_LOJ_75

TC_LOJ_76

TC_LOJ_80

TC_LOJ_81

TC_LOJ_82

TC_LOJ_85

TC_LOJ_86

TC_LOJ_87

TC_LOJ_88

TC_LOJ_89

TC_LOJ_91

TC_LOJ 93

TC_LOJ_97

TC_LOJ_99

TC_LOJ_100

TC_LOJ_102

TC_LOJ_103

TC_LOJ_104

TC_LOJ_107

TC_LOJ_108

TC LOJ 109

TC_LOJ_111

TC_LOJ_114

TC_LOJ_116

TC_LOJ_117

TC_LOJ 118

TC_LOJ_119

TC_LOJ_120

TC_LOJ 121

TC_LOJ_122 chr1:1413411-1413530 chr23:504383-504519 chr23:505516-505635 chr1:2018618-2018750 chr37:132370-132499 chr13:741015-741134 chr1:351420-351541 chr18:746701-746824 chr37:464692-464819 chr16:213322-213477 chr2:121560-121715 chr12:107750-107877 chr27:329031-329151 chr26:38201-38343 chr33:297174-297306 chr26:479107-479233 chr11:853646-853766 chr13:783091-783210 chr15:807734-807870 chr2:160058-160182 chr13:664297-664421 chr26:419336-419479 chr41:288290-288430 chr5:168922-169061 chr26:336772-336902 chr3:965641-965793 chr15:398374-398497 chr1:2137512-213763 chr3:196127-196261 chr27:93351-93474 chr36:377593-377718
ACACTGACGACATGGTTCTACACAAGATTGTTCCACTGACGAAGACA ACACTGACGACATGGTTCTACACTTCATCATCTATGCTCCGACGAC ACACTGACGACATGGTTCTACAGTGGACCCAAATGTACTCAGCAAC ACACTGACGACATGGTTCTACAAGTGGACATGGTGACGAAGATGAG ACACTGACGACATGGTTCTACAACCGGATGTATTCCTCTCGTGGTA ACACTGACGACATGGTTCTACACACAAACCGCTTAGACCCTGAAGT ACACTGACGACATGGTTCTACAAGACTCAATCGCCTTCACGACATA ACACTGACGACATGGTTCTACAACCCACTCCAGTAGCATTTCTTCC ACACTGACGACATGGTTCTACACAGATGCTGCCTTGACAGAGATGTA ACACTGACGACATGGTTCTACAGTAAATAGACACAAGCCATTCCCATC ACACTGACGACATGGTTCTACACTCATACCCTTGCTTTGTCATGCT ACACTGACGACATGGTTCTACAGAATGACAACAATGCCCTTTCTTC ACACTGACGACATGGTTCTACATCGTAAAGGTATTGGGCATATTCG ACACTGACGACATGGTTCTACATTTGAAGAGAAGATGGCCCTGAGT ACACTGACGACATGGTTCTACACAAGTTCCTGTTGGACGTGGTAGT ACACTGACGACATGGTTCTACATATTATTTACGAAACGGCGGAGGA ACACTGACGACATGGTTCTACAAGAACAGGAAGTTTGTGACGGTTG ACACTGACGACATGGTTCTACAGTACACCCGTCCTTGCAGTATGATT ACACTGACGACATGGTTCTACACAAGTTCGCAATGTAGGAAAGCTG ACACTGACGACATGGTTCTACAGTCATACCTTACCAAACGGCACAG ACACTGACGACATGGTTCTACATATCTGTGGTGGCTGTAGATGGTG ACACTGACGACATGGTTCTACACTTTCGGTGTTACGGTGTACTTCAG ACACTGACGACATGGTTCTACACCACGCCACCAGTAACGATAATAA ACACTGACGACATGGTTCTACATTAGAAACCGTGTAGAGACTTGTCAGC ACACTGACGACATGGTTCTACAGCTGTCTCCAAGAGTCGCAGAATA ACACTGACGACATGGTTCTACATCCAATCTCTTATCTTTCAGGAGAACG ACACTGACGACATGGTTCTACACCACAAGTAGGCTGAACCACAAAT ACACTGACGACATGGTTCTACAGAATCATCAGAGGGTCATTTGCAC ACACTGACGACATGGTTCTACATCATCCTCATCTTCTGGTGGTGAT ACACTGACGACATGGTTCTACAACTGCGTTGTATAGCCGAATCACT ACACTGACGACATGGTTCTACACTTTCCTGGGTTCGTTGGTTTAAG
TACGGTAGCAGAGACTTGGTCTTTTGAGAGCGTGAAGGAGTACACA TACGGTAGCAGAGACTTGGTCTTCTGAATGACTGGTTGAAAGACGA TACGGTAGCAGAGACTTGGTCTGAACCTAAGAAACGAAGAACCCTCA TACGGTAGCAGAGACTTGGTCTGTAGTGCTTCAAACCGCTCAAGAA TACGGTAGCAGAGACTTGGTCTCATGCACTTATCGTCGTCACTTTC TACGGTAGCAGAGACTTGGTCTCCAGAAGAAACAATCAATCAACAGC TACGGTAGCAGAGACTTGGTCTCAGAGGTGTTTATGAGCAAGTACCG TACGGTAGCAGAGACTTGGTCTTTAACTATGGCAATGAGGCAGAGC TACGGTAGCAGAGACTTGGTCTACGAGTGTAGAAGCGAAGATGCTG TACGGTAGCAGAGACTTGGTCTTACTATCACTACCGTGGGCGTCAG TACGGTAGCAGAGACTTGGTCTGTTCAGGAGACGGACCACTAGGTT TACGGTAGCAGAGACTTGGTCTGTATCTCCATCCATTTCCCAGTGC TACGGTAGCAGAGACTTGGTCTCCAGGATCATTCAGCTTAGTCCAG TACGGTAGCAGAGACTTGGTCTTTGAAGAAAGGATCTGCCTCGTAA TACGGTAGCAGAGACTTGGTCTAATGTACGCAAGGAGCGACTAGAG TACGGTAGCAGAGACTTGGTCTAGGAGATGGCTCACTCACTTGAAC TACGGTAGCAGAGACTTGGTCTATCACCTCTGAAAGAATCGACTGC TACGGTAGCAGAGACTTGGTCTCGCTGAGTTCACGAAGTTATGCTT TACGGTAGCAGAGACTTGGTCTTATCATGGTGGTCGATGCTGAATA TACGGTAGCAGAGACTTGGTCTATGTGAACAACCGTACTGGAGGTG TACGGTAGCAGAGACTTGGTCTCGACGACAACAAGGAAGAAGAGGTA TACGGTAGCAGAGACTTGGTCTTCACTGTTTACAACTACGGCCAGA TACGGTAGCAGAGACTTGGTCTGAAGAAGTGGTACTCTCCCGATCC TACGGTAGCAGAGACTTGGTCTATTACCCTGCACCAAGACACATTC TACGGTAGCAGAGACTTGGTCTCATGGATTTCTTTCCAGTGCTTTG TACGGTAGCAGAGACTTGGTCTCATACTCAAACGAGGCACGAATCT TACGGTAGCAGAGACTTGGTCTGTCAAGCCCTTCGTATCCCTGTTA TACGGTAGCAGAGACTTGGTCTAGTACACAACAAAGTTATCGCGGATG TACGGTAGCAGAGACTTGGTCTTGGACTCTCACTTCTGTATCTACTTTGTTG TACGGTAGCAGAGACTTGGTCTGACAGGAACACCAAATGTACTGTGAA TACGGTAGCAGAGACTTGGTCTCAGGTGTTCCTCGTCAAGCTGTAAT 
Supplementary Table 2 (continued)

TC_LOJ_124

TC_LOJ_125

TC_LOJ_126

TC_LOJ_128

TC_LOJ_129

TC_LOJ_130

TC_LOJ_131

TC_LOJ_136

TC_LOJ_137

TC_LOJ_138

TC_LOJ_140

TC_LOJ_141

TC LOJ 142

TC_LOJ_144

TC_LOJ_145

TC_LOJ_146

TC_LOJ_147

TC_LOJ_152

TC_LOJ_154

TC_LOJ_156

TC_LOJ_157

TC_LOJ_158

TC_LOJ_159

TC_LOJ_160

TC_LOJ_161

TC_LOJ_162

TC LOJ 163

TC_LOJ_165

TC_LOJ_166

TC_LOJ_168

TC_LOJ_169 chr10:933564-933686 chr21:539837-539959 chr15:908929-909068 chr11:775649-775772 chr18:115349-115471 chr9:601749-601872 chr9:601909-602028 chr23:522688-522812 chr16:889485-889604 chr5:1116604-1116723 chr19:251999-252118 chr37:317244-317399 chr2:327727-327846 chr11:235518-235637 chr6:23502-23628 chr27:232849-232974 chr4:1219111-1219233 chr19:553417-553540 chr37:156377-156496 chr5:627080-627199 chr1:1963178-1963304 chr1:1964699-1964825 chr1:1998360-1998510 chr16:738527-738679 chr43:149662-149786 chr16:189968-190097 chr18:523652-523773 chr3:169504-169625 chr3:169646-169792 chr28:364521-364659 chr11:721966-722086
ACACTGACGACATGGTTCTACATGCAAATACAGAAGATGAGCTACGC ACACTGACGACATGGTTCTACAAAATCTCAGCTACAACAACATCTCTGG ACACTGACGACATGGTTCTACAGGCCTTCTCACTAACTGTCGATCTG ACACTGACGACATGGTTCTACAGAAAGAAGCTGAAGAATGGGCAAA ACACTGACGACATGGTTCTACAGTGACTTGGCGATTATGATTCGTT ACACTGACGACATGGTTCTACATCCCGTTACATCCAATACATCCAA ACACTGACGACATGGTTCTACAACAAGCAATCCAATTACAACCACAG ACACTGACGACATGGTTCTACATTTCAAGCTGCGACTTAATCAACG ACACTGACGACATGGTTCTACACATTTCTGCTGCTTCCTTTGAGAA ACACTGACGACATGGTTCTACACATTTCACCAGAAGTGACAGCAAC ACACTGACGACATGGTTCTACACCCTCACCTCAATCATATCCACAC ACACTGACGACATGGTTCTACAATTGTGAGAGGATGGGTTCAAATG ACACTGACGACATGGTTCTACAATGCGGGAGTGTTGTGCATTAGTAT ACACTGACGACATGGTTCTACAACGCAGTTGGTCGAGAATTGTATC ACACTGACGACATGGTTCTACATTGGCATAAAGGTACGAATCATGG ACACTGACGACATGGTTCTACACTCAGTATGAACTCCGCTTCCTGT ACACTGACGACATGGTTCTACAAAGCTGAATAGATCGCACAAGCTC ACACTGACGACATGGTTCTACACATAAGGGCAGTGTCATCAACAAA ACACTGACGACATGGTTCTACAGTAAGGACCACAAGAGGGAAATGG ACACTGACGACATGGTTCTACATGGACTACGAGAAGGTTTCATACGAC ACACTGACGACATGGTTCTACATAGAAGAGCGTGTGAAGACTGTGG ACACTGACGACATGGTTCTACACTACACGCATTGTGAGAAACTTGG ACACTGACGACATGGTTCTACAACCGTGCTACTTTCTTCCTTTGGT ACACTGACGACATGGTTCTACACAGCCACTGTTCAGATCCACAAGT ACACTGACGACATGGTTCTACATGTACCTTTCTGCTTTGTCTTCTTCC ACACTGACGACATGGTTCTACAGCTTTGGAGTAGAGCAGATTTGGA ACACTGACGACATGGTTCTACAGATCGCGTTGTAAGCAAATTCAAG ACACTGACGACATGGTTCTACACACGAAAGTCAAACTCCTCCACAA ACACTGACGACATGGTTCTACAGGCAACGTGGTATGGAATGATAAC ACACTGACGACATGGTTCTACACTCGTGGAAGTTTAGTGCTGATCG ACACTGACGACATGGTTCTACAATGAAACACGTATGCACGATATGC
TACGGTAGCAGAGACTTGGTCTTGATTATGAGGAGGAGGATGCAGT TACGGTAGCAGAGACTTGGTCTTCATCCTTTCCATCGTTCTCACTT TACGGTAGCAGAGACTTGGTCTACCTTCTTATCACGGAAGAGTATCAGG TACGGTAGCAGAGACTTGGTCTGTTGATCCTGGCAATTACACTCGT TACGGTAGCAGAGACTTGGTCTCGTTTGTCTTCTCATCCTTCTTCG TACGGTAGCAGAGACTTGGTCTTGCATACACAACAGAGCTAAGTGTCG TACGGTAGCAGAGACTTGGTCTATTAAAGAAGGTCGCGGCAGTAGA TACGGTAGCAGAGACTTGGTCTGATGGAAATGCTTCTTGCACAGTC TACGGTAGCAGAGACTTGGTCTTCTGATGTTGATCTCTCTTTAACCTACCG TACGGTAGCAGAGACTTGGTCTGATGAGGGAGAAGCGAATTTGAAC TACGGTAGCAGAGACTTGGTCTGGGACAAGTACGGGAACAGAATAGA TACGGTAGCAGAGACTTGGTCTCCAGTGCATACTTCTGTGTTATGGTAGA TACGGTAGCAGAGACTTGGTCTACGGAATACGGGTGGAATAAGAAA TACGGTAGCAGAGACTTGGTCTGAAGGAGAGGTGGTGCAGCTTATC TACGGTAGCAGAGACTTGGTCTGAACTCACGACCCTGAATAAGACG TACGGTAGCAGAGACTTGGTCTGGATATGTGCTCAAAGTGCCTTGT TACGGTAGCAGAGACTTGGTCTTATGCCCTATCCGTGTTTCTTACG TACGGTAGCAGAGACTTGGTCTGTATTGCTGGTTGGTTCTCTTCCA TACGGTAGCAGAGACTTGGTCTGCAGAGTAGACAGCATGGAGTGTG TACGGTAGCAGAGACTTGGTCTGCTGTGGAAATGTTGTGATCCTGT TACGGTAGCAGAGACTTGGTCTATGACAACCGCGTCACTTGAATAC TACGGTAGCAGAGACTTGGTCTTGAATTTGTCTGGGATGTGGAAAC TACGGTAGCAGAGACTTGGTCTAATCTTCCTCAATCTCCCTGCTGT TACGGTAGCAGAGACTTGGTCTGGCACAAGACCATCAAAGTAGGAC TACGGTAGCAGAGACTTGGTCTTGATGACTATCGCTCCATTCTTCC TACGGTAGCAGAGACTTGGTCTCCGAGTTACATTTCTTTGCCTTTG TACGGTAGCAGAGACTTGGTCTGGCGTAAAGGGCAACTCAAAGTAT TACGGTAGCAGAGACTTGGTCTGGTAAATACACGTCCACCGACCTT TACGGTAGCAGAGACTTGGTCTTCTGCTCACACAGGACTGAATCTC TACGGTAGCAGAGACTTGGTCTCGATGATAAAGAAGTCTCCGTACCC TACGGTAGCAGAGACTTGGTCTGGCGCTAAATCTGTACGAATACCA 
Supplementary Table 2 (continued)

TC_LOJ_170

TC_LOJ_171

TC_LOJ_173

TC_LOJ_174

TC_LOJ_175

TC_LOJ_177

TC_LOJ_178

TC_LOJ_180

TC_LOJ_181

TC_LOJ_182

TC_LOJ_184

TC_LOJ_185

TC LOJ 187

TC_LOJ_188

TC_LOJ_191

TC_LOJ_192

TC_LOJ_195

TC_LOJ_197

TC_LOJ_200

TC_LOJ_201

TC_LOJ_203

TC_LOJ_204

TC_LOJ_205

TC_LOJ_206

TC LOJ 209

TC_LOJ211

TC LOJ 212

TC_LOJ_213

TC_LOJ 214

TC_LOJ_215

TC_LOJ_217 chr36:416713-416839 chr2:854454-854583 chr19:264153-264279 chr18:456154-456275 chr13:608121-608257 chr7:1112127-1112263 chr10:265161-265291 chr8:851024-851146 chr7:987164-987292 chr15:497344-497472 chr37:138690-138820 chr27:387192-387314 chr15:795497-795621 chr1:2220221-2220341 chr5:703969-704096 chr37:447759-447878 chr27:40705-40826 chr41:298702-298834 chr37:173415-173536 chr32:855499-855637 chr25:64845-64984 chr9:194610-194758 chr7:1037003-1037155 chr19:762223-762346 chr1:2005883-2006014 chr2:916287-916407 chr44:285730-285879 chr32:839358-839478 chr11:849661-849797 chr10:1052122-1052245 chr1:2773733-2773861
ACACTGACGACATGGTTCTACAGGGAGTACGAGTTTGCAGAGAAGA ACACTGACGACATGGTTCTACAAGCAAGGGCAGTCACAAAGTAACA ACACTGACGACATGGTTCTACACATTGAGAACCACGACTGGCTATT ACACTGACGACATGGTTCTACAATATCATGGGACTTGCCGGATTAC ACACTGACGACATGGTTCTACAACTGACATGGATCATAGCCAATCG ACACTGACGACATGGTTCTACACTTTGAGAGCTTTGCATCCTTCAC ACACTGACGACATGGTTCTACAGGTATGAGCATCGCCTTATTGATG ACACTGACGACATGGTTCTACAGACGATGAGGAGTTGGAGGATGTA ACACTGACGACATGGTTCTACATAGATGTTTGGTCCCATTTGAAGG ACACTGACGACATGGTTCTACATGTCCAAGACCTTCACATAGTCCA ACACTGACGACATGGTTCTACAAGCTTGGCCTTCAACACATCATTA ACACTGACGACATGGTTCTACAGGGTGATAGATGCTGTTGCTGAAT ACACTGACGACATGGTTCTACAGACAAACATTCGACCTTCATCTTCTG ACACTGACGACATGGTTCTACACCAGGTTGTTGGTTGTTATGTGGT ACACTGACGACATGGTTCTACACTATTGGATGGGAACGTGGTACAG ACACTGACGACATGGTTCTACACGTATCAAACAGGGCTGGAGACTT ACACTGACGACATGGTTCTACAATGTTTCCTTGCATGAGTTTGTGG ACACTGACGACATGGTTCTACAATTGGGACGGTAGAGCATGTAAGG ACACTGACGACATGGTTCTACACACGAAACTGCCAATGATGACTCT ACACTGACGACATGGTTCTACAAAGAGGCGTGTAAGAAGTATGTGGAG ACACTGACGACATGGTTCTACAACGCGGATACTAGGGAACATGAGT ACACTGACGACATGGTTCTACACTGTTCAAAGTCCATTGTGCTATCC ACACTGACGACATGGTTCTACAACAGGGCTTCAGGTGGACATTATT ACACTGACGACATGGTTCTACAAGCCTTCCCTTTCTACTGGTGGTA ACACTGACGACATGGTTCTACATCTTTGAAGGTTCTGGTGTTGGTT ACACTGACGACATGGTTCTACACTTGATAAACTCTGCGGCTTCCTC ACACTGACGACATGGTTCTACAGCTGTCCATATCCGCATCTTCTAA ACACTGACGACATGGTTCTACAGGTGACAAACCCATTCAGCTTACA ACACTGACGACATGGTTCTACATTACTACATTGGTGGCGAGACAAAC ACACTGACGACATGGTTCTACACAGAGTTCTACAAGGAAGATCGACAAA ACACTGACGACATGGTTCTACAAAACTTATGGCGTACAACAGGGAGT
TACGGTAGCAGAGACTTGGTCTAGAGGGTTGACATAAGGATGCAGA TACGGTAGCAGAGACTTGGTCTACTGTGGGTGATACAGGCAAAGAC TACGGTAGCAGAGACTTGGTCTGGACTATGAGATCGACAAGGAGTTTG TACGGTAGCAGAGACTTGGTCTCAATGTCTGGTTTGGAGGAAGAAG TACGGTAGCAGAGACTTGGTCTCGATAAAGGAACCCAACAAGAACC TACGGTAGCAGAGACTTGGTCTCCGGGACGAGTACACATATACCAA TACGGTAGCAGAGACTTGGTCTAAGAGAACCAAATCCCTGAGCAAC TACGGTAGCAGAGACTTGGTCTAGTGTGGCGATAGGTGATTGTGAT TACGGTAGCAGAGACTTGGTCTTGATACCGTCACTATTACCGCTAGAAA TACGGTAGCAGAGACTTGGTCTTGGTTACTTTCCAGACAAGGGATG TACGGTAGCAGAGACTTGGTCTGCGTCATACTCCCTCACATATCCA TACGGTAGCAGAGACTTGGTCTTGAGTTTAATGGACCCGAAGGAAC TACGGTAGCAGAGACTTGGTCTTGGTATTTGAGGATCATTCCAGTCA TACGGTAGCAGAGACTTGGTCTGCGGAGATTCACGAAATAGAGGAA TACGGTAGCAGAGACTTGGTCTGCACAATCTCTGTTGTAAGACTAAACTCCT TACGGTAGCAGAGACTTGGTCTATCAAGCTGCAAGAAGAGAACATCC TACGGTAGCAGAGACTTGGTCTGGAGTCGCCGTAGTATTCCCTTATG TACGGTAGCAGAGACTTGGTCTGCCTGAGTTCCTCCAGTCTTTCTT TACGGTAGCAGAGACTTGGTCTCACCTCCGTCTTTCTTCTCCTTCT TACGGTAGCAGAGACTTGGTCTTGCAAGTAGTCAGCAATGTCCAGT TACGGTAGCAGAGACTTGGTCTTTGAGCAGAATACCAAAGCAGTTGT TACGGTAGCAGAGACTTGGTCTATGACTGCAAGGTATTCCGCTTCT TACGGTAGCAGAGACTTGGTCTGGTTAAAGGTCGTGGTTGACACAT TACGGTAGCAGAGACTTGGTCTTCTGATTTCATACACGTTGCTCCTC TACGGTAGCAGAGACTTGGTCTTCTCAGGGACGAGGAGACATATAAGA TACGGTAGCAGAGACTTGGTCTCAATGGTACGAACATGATTGACTGTG TACGGTAGCAGAGACTTGGTCTATGTCGTTTCCAAATCAGCACAAC TACGGTAGCAGAGACTTGGTCTTACAGCGCCAATCAAATCCACTAC TACGGTAGCAGAGACTTGGTCTTCAGACGAAACAGATAGCTCGTGA TACGGTAGCAGAGACTTGGTCTTTAATGATGGGTGGAAGTGAGAGG TACGGTAGCAGAGACTTGGTCTCGATAACGACGATGAAGATGATGA 
Supplementary Table 2 (continued)

TC_LOJ_219 TC_LOJ_220 TC_LOJ_221 TC_LOJ_223 TC_LOJ_224 TC_LOJ_225 TC_LOJ_227 TC LOJ 228 TC_LOJ_229 TC LOJ 230 TC_LOJ 231 TC_LOJ_232 TC LOJ 234 TC_LOJ_235 TC_LOJ_236 TC_LOJ_237 TC_LOJ_238 TC_LOJ_239 TC_LOJ_242 TC_LOJ_243 TC_LOJ_244 TC_LOJ_245 TC_LOJ_249 TC_LOJ 250 TC LOJ 251 TC_LOJ_252 TC_LOJ_253 TC_LOJ_255 TC_LOJ_256 TC_LOJ_257 TC_LOJ_259 chr26:38066-38187 chr14:923562-923682 chr11:868950-869070 chr27:96137-96258 chr1:2775484-2775623 chr15:246311-246435 chr27:116142-116263 chr5:1147485-1147616 chr5:1148049-1148168 chr15:926778-926915 chr1:2138077-2138196 chr5:191326-191447 chr10:715504-715626 chr15:197505-197635 chr11:235245-235379 chr9:134209-134328 chr21:322787-322911 chr44:237246-237373 chr31:92921-93071 chr21:288200-288319 chr18:566462-566592 chr3:1209990-1210114 chr10:1031977-1032097 chr21:505080-505199 chr5:743274-743396 chr36:237339-237479 chr3:240382-240505 chr27:388555-388675 chr39:221720-221854 chr5:992280-992407 chr32:837402-837557
ACACTGACGACATGGTTCTACAGTTGATGTGGATAGGCTTGACTACTTTC ACACTGACGACATGGTTCTACATCGGGTAAATGTCTAACGGAGAAA ACACTGACGACATGGTTCTACAGCTTCACAGCTATCGAGGTGTATTG ACACTGACGACATGGTTCTACACAAGCGCACCCTAATAAGAAATTG ACACTGACGACATGGTTCTACAGGTGTGTACGGATGACTGCTACTTACTT ACACTGACGACATGGTTCTACACGTGAAAGATACGGCTGACACATA ACACTGACGACATGGTTCTACAATGAGGAGGAGGAGAAATGGAAAC ACACTGACGACATGGTTCTACAACAGTGCAGTCGTACTTTCGCATT ACACTGACGACATGGTTCTACAAGTGGCTTGGCAGATTTCTTCTGT ACACTGACGACATGGTTCTACAATTCTGCCTGCGACAGTAGTTCTC ACACTGACGACATGGTTCTACAGGCAGACTCCAGATACTGACGAAT ACACTGACGACATGGTTCTACAACATCCTGACCCTTGGCTTTAGAC ACACTGACGACATGGTTCTACAAGTAAGCCTGTTGCTTTGGAAACTC ACACTGACGACATGGTTCTACATCGTCAATTTCCCGTAGGATACTTT ACACTGACGACATGGTTCTACAATCTTTACCATGCACCTCCACAAC ACACTGACGACATGGTTCTACACTCTTCACGCCAATACATTCCTTG ACACTGACGACATGGTTCTACATCAGGGTAGATTCATCAGGCAGAG ACACTGACGACATGGTTCTACAATTTATGCCCGCAAACCAGATAAC ACACTGACGACATGGTTCTACAATTGAAGTATCGCCAGAACAGCAT ACACTGACGACATGGTTCTACACGGTCAGGATCGTTATAGTTTGGTAG ACACTGACGACATGGTTCTACAATTATCTCGTGAGTTTGGCGGAAT ACACTGACGACATGGTTCTACAGGATCGACGTATGGGACGTATTTC ACACTGACGACATGGTTCTACAAAGCTCAGTGTTCAAAGTGCCATC ACACTGACGACATGGTTCTACAGTTCTCCGTTACTTTCCGACACAG ACACTGACGACATGGTTCTACACTAGGGATAGTGTCTCAACATTGGCTATAA ACACTGACGACATGGTTCTACATTAGAGCTTCGTATCGGCATGTTG ACACTGACGACATGGTTCTACACCACTACCATTACCCGTGTCGTTA ACACTGACGACATGGTTCTACAGTTATTTGTATCCGTATCTTGCTGTCG ACACTGACGACATGGTTCTACAAACTGACCGGAAGTGAGATTGATG ACACTGACGACATGGTTCTACACCTTTATTACGCTTCGGCAAGTACA ACACTGACGACATGGTTCTACAACTCTACACAAAGGCGTCAGAGATG
TACGGTAGCAGAGACTTGGTCTTCACCTTCGTAGCACAATACCTTACA TACGGTAGCAGAGACTTGGTCTCCAGATCCAGTGATTCGTCTTGTT TACGGTAGCAGAGACTTGGTCTCCAGGAGTTTAGTTACAACAGACGAGA TACGGTAGCAGAGACTTGGTCTCAACAAAGAGCTTCAAATGGTGTG TACGGTAGCAGAGACTTGGTCTCAACAAGGACAAAGACAACCACAA TACGGTAGCAGAGACTTGGTCTGTAGTGCGTGTTGCTCCTGTTGTT TACGGTAGCAGAGACTTGGTCTGTCGATGACACAGTCCAGACACTC TACGGTAGCAGAGACTTGGTCTTGTTGACTACTTTGACGGAAATCGT TACGGTAGCAGAGACTTGGTCTTGACAGTTTAGAGAGCGTTGTAGTGAAAG TACGGTAGCAGAGACTTGGTCTCCATTCTTCGTGAAATTGAGGTTG TACGGTAGCAGAGACTTGGTCTCCACAACTCCTTGACGACTTTCTT TACGGTAGCAGAGACTTGGTCTGGTTAGAGAGAACATTACGACGGAGA TACGGTAGCAGAGACTTGGTCTTCAACCCAGACGAAAGTCTAGTGG TACGGTAGCAGAGACTTGGTCTCAGGAGGAGGGTGAACTGATAATG TACGGTAGCAGAGACTTGGTCTGGTCTCACCACGTATCACGAGAAG TACGGTAGCAGAGACTTGGTCTCCAGCTACAACTGCAAACAAATACAC TACGGTAGCAGAGACTTGGTCTTATCAACAATGCTCGACACCCACT TACGGTAGCAGAGACTTGGTCTCGAGGCAATTCGTATAATGTCTTCA TACGGTAGCAGAGACTTGGTCTGTGTTGCTTGGAGTAAGGCACTCT TACGGTAGCAGAGACTTGGTCTAGACACTTTGTATCGTATGCGTCGT TACGGTAGCAGAGACTTGGTCTCAGAACCGTCTTGTCCTTCACTTC TACGGTAGCAGAGACTTGGTCTTTGAAGGACTGGAGCAAGACAAGT TACGGTAGCAGAGACTTGGTCTTTTCCTTGTTATCGGCTGTGAGAA TACGGTAGCAGAGACTTGGTCTTGCCATGTTACCCATAAACCACTT TACGGTAGCAGAGACTTGGTCTCACCCTTTAACTTTGAACGAACACG TACGGTAGCAGAGACTTGGTCTCACTTCATACATTTCCTCCAGAGACC TACGGTAGCAGAGACTTGGTCTCGCAGTCCTTGCTTAACCTCATTT TACGGTAGCAGAGACTTGGTCTAGTATCACCTGGAGGACCGTGAAG TACGGTAGCAGAGACTTGGTCTGGGCGGCGTCGTAGTATAAATAAG TACGGTAGCAGAGACTTGGTCTTTCCACGCAAACAATCAGTATCAG TACGGTAGCAGAGACTTGGTCTCCTGCAAGATCAATAAGGTTCAGC 
Supplementary Table 2 (continued)

TC_LOJ_260

TC_LOJ_262

TC_LOJ_264

TC_LOJ_265

TC_LOJ_266

TC_LOJ_267

TC_LOJ_268

TC LOJ 269

TC_LOJ_271

TC_LOJ_273

TC_LOJ 274

TC_LOJ_275

TC LOJ 276

TC_LOJ_277

TC_LOJ_278

TC LOJ 279

TC_LOJ_280

TC_LOJ_281

TC_LOJ_282

TC_LOJ_283

TC_LOJ_285

TC LOJ 286 chr4:1353006-1353141 chr1:2151183-2151303 chr18:649186-649316 chr27:343910-344029 chr1:2205081-2205202 chr26:405401-405543 chr26:302445-302583 chr5:495739-495879 chr2:323827-323957 chr1:2140290-2140430 chr21:239185-239310 chr39:50470-50598 chr1:2694842-2694979 chr4:1382610-1382749 chr2:164952-165077 chr9:400076-400197 chr21:505326-505445 chr10:735827-735952 chr37:470203-470342 chr12:561576-561706 chr31:132291-132424 chr15:941983-942118
ACACTGACGACATGGTTCTACATGGTACTTGTTCAGCTCGGAAATC ACACTGACGACATGGTTCTACACCGTAGTTGCGGTACGAATAAGTG ACACTGACGACATGGTTCTACAGTGGAGGCGAAGAAGAAGTTTACA ACACTGACGACATGGTTCTACAGTGCATCATATTCGATAGGGAGATGT ACACTGACGACATGGTTCTACACTACGAAGTGCCTTAACTGCCTCA ACACTGACGACATGGTTCTACATTGCTTTCGATGGAGATAGACCTTT ACACTGACGACATGGTTCTACACGTAGTCAAACGGACTGAAGTACACA ACACTGACGACATGGTTCTACATCTTTATGACAAGTGCAACCAAAGC ACACTGACGACATGGTTCTACAGTGGGTTTCATCTCTCGTTTATGC ACACTGACGACATGGTTCTACACAATGGCACCAAGATAATAGTACAGGA ACACTGACGACATGGTTCTACAAACAAGGTGAAGAAGAGCCATCAG ACACTGACGACATGGTTCTACACTGCTCCTGATACTGCACAAACTG ACACTGACGACATGGTTCTACATTACACATTGCAGGGCAGCATATT ACACTGACGACATGGTTCTACATAGCATCTTAATCAGCTCGGGAGA ACACTGACGACATGGTTCTACAGGTCATTCACGCCAGTTCATACAT ACACTGACGACATGGTTCTACACGAGACAGGGATGGACTCTTCAAT ACACTGACGACATGGTTCTACAGATTGCTACGTGAAGACGTGGAAG ACACTGACGACATGGTTCTACACAACGCATTTGGATTGCCTACTAA ACACTGACGACATGGTTCTACACTACTCAAGGAACCAGGCGTATTG ACACTGACGACATGGTTCTACACAGAAGGAGAAGACATTGGAACTCA ACACTGACGACATGGTTCTACACATTGACCTTGCCACAGAAGTGTA ACACTGACGACATGGTTCTACAGGCGTATCCACCACAAGAGTAGAA
TACGGTAGCAGAGACTTGGTCTCAAAGGCAGAGGAATGTTCAAAGA TACGGTAGCAGAGACTTGGTCTACTGGGAACGTGTATTAGGTATGGAGT TACGGTAGCAGAGACTTGGTCTAATAGAAACGGCATTCCATAAGCAC TACGGTAGCAGAGACTTGGTCTTATTACAGCATTGACCGTGTCTTCC TACGGTAGCAGAGACTTGGTCTATTCTATGTGCGTTTGGGTTTCAG TACGGTAGCAGAGACTTGGTCTGCGGAGATGTCTGATTTAGGAATTG TACGGTAGCAGAGACTTGGTCTGAGGAGGCAGTGGAGGTGTTAAAT TACGGTAGCAGAGACTTGGTCTCGTGATACTCCACCGTCTCAATCT TACGGTAGCAGAGACTTGGTCTACCCTTGTCCATGTGTCTTGTAGC TACGGTAGCAGAGACTTGGTCTTGCAGAACCATCGTGAGAACTTTA TACGGTAGCAGAGACTTGGTCTAAGGTGGAGGAGTTTGAACAGTACG TACGGTAGCAGAGACTTGGTCTGGTGCCTACAATGACTCCGTACAC TACGGTAGCAGAGACTTGGTCTGTCTTTGTTCGTCATGTCAGCGTA TACGGTAGCAGAGACTTGGTCTGACGAACAAATGGAGAATCAGACG TACGGTAGCAGAGACTTGGTCTACGGCCTTCTTCATAATCTCCATAA TACGGTAGCAGAGACTTGGTCTGTTACGATGGCCTTGAGTGTGAGA TACGGTAGCAGAGACTTGGTCTGAGCGTATCGTACAGGCCAAAGTA TACGGTAGCAGAGACTTGGTCTAAACGTCTTGGTCTGTACGAGGAG TACGGTAGCAGAGACTTGGTCTAACGTCCCACCAAGAATAATGAGC TACGGTAGCAGAGACTTGGTCTTCTTTGCCACTATCAAGCACCAAC TACGGTAGCAGAGACTTGGTCTTGGCCTTATTCACATACTCCACAAG TACGGTAGCAGAGACTTGGTCTGGATGCCAGATTACGTGAAAGAAA 
Supplementary Table 3 Summary of GLST library preparation and sequencing costs. Green dots indicate items/costs related to first-round PCR and clean-up. Blue dots indicate items/costs related to barcoding PCR and clean-up. The cost summary does not consider qPCR materials because we applied qPCR only for purposes of method development. It is not essential for GLST. Abbreviations: EUG Eurofins Genomics); NEB (New England Biolabs); MGRD (median genotype read-depth).

Item

200 GLST primer primer pairs (EUG) Q5 High-Fidelity 2X Master Mix (NEB) •

UltraPure Agarose (Invitrogen) •

100 bp DNA Ladder* (NEB) •

$6 \mathrm{X}$ Gel Loading Dye (NEB) •

PureLink Quick Gel Extraction Kit (Invitrogen) •

SYBR Safe (Invitrogen) •

$$
\text { Miscellaneous • }
$$

Barcoded reverse primer (EUG) •

Universal forward primer (EUG) •

Q5 High-Fidelity 2X Master Mix (NEB) •

Nuclease-free $\mathrm{dH}_{2} \mathrm{O}$ (Qiagen) •

Qubit assay tubes (Invitrogen) •

Qubit dsDNA HS Assay Kit (Invitrogen) •

UltraPure Agarose (Invitrogen) •

100 bp DNA Ladder (NEB) •

$6 \mathrm{X}$ Gel Loading Dye (NEB) •

PureLink Quick Gel Extraction Kit (Invitrogen) • SYBR Safe (Invitrogen) •

$$
\text { Miscellaneous • }
$$

Illumina Reagent Kit v2 Micro 300-cycle Illumina MiSeq

\section{Availability}

$60.90 \mathrm{ml} / 1508.88 £$

$2.5 \mathrm{ml} / 106.75 £$

$100 \mathrm{~g} / 124.00 £$

$50 \mathrm{ug} / 34.50 £$

$1 \mathrm{ml}$ comes free with ladder ${ }^{*}$

$3 \times 50$ units / $143.64 £$

$400 \mu \mathrm{l} / 62.78 £$

$\mathrm{n} / \mathrm{a}$

$0.02 \mu \mathrm{mol} / 49.95 £$

$0.02 \mu \mathrm{mol} / 49.95 £$

(see above)

$1000 \mathrm{ml} / 35.68 \varepsilon$

500 tubes $/ 51.50 £$

100 assay kit / $66.25 £$

(see above)

(see above)

(see above)

(see above)

(see above)

$\mathrm{n} / \mathrm{a}$ (quantity / price)

Quantity for
100 samples
$25 \mathrm{pmol}$
$500 \mu \mathrm{l}$
$15.6 \mathrm{~g}$
$13 \mathrm{ug}$
$226 \mu \mathrm{l}$
$100 \mathrm{units}$
$60 \mu \mathrm{l}$
$\mathrm{n} / \mathrm{a}$
$0.8 \mathrm{nmol}$
$0.8 \mathrm{nmol}$
$1 \mathrm{ml}$
$540 \mu \mathrm{l}$
102 tubes
100 assays
$1.2 \mathrm{~g}$
$1 \mathrm{ug}$
$9 \mu \mathrm{l}$
$1 \mathrm{unit}$
$10 \mu \mathrm{l}$
$\mathrm{n} / \mathrm{a}$

Cost for

100 samples

$21.35 £$

$19.34 £ 13$ agarose gels $(0.8 \%)$ to visualize 100 samples, separated by empty lanes

$8.97 £ 0.5 \mathrm{ug}$ ladder at left and right margins of each gel

$0.00 £ 2 \mu \mathrm{l}$ dye for each sample/ladder lane

$95.76 £$

$9.42 £$

$50.00 £$ Pipette tips, vials, blades, etc.

$2.00 £$ Primers purified by manufacturer using high performance liquid chromatography

$2.00 £$ Primers purified by manufacturer using high performance liquid chromatography

$42.70 £$

$19.27 £$

$10.51 £$

$66.25 £$

$1.49 £$ Only one agarose gel $(0.8 \%)$ is needed because samples have been pooled

$0.69 £ \quad 0.5 \mathrm{ug}$ ladder at left and right margins of the gel

$0.00 £ 7 \mu \mathrm{l}$ dye for sample (pool) lane, $2 \mu \mathrm{l}$ for each ladder lane

$0.96 £$ Only one unit is needed because samples have been pooled

$1.57 £$

$50.00 £$ Pipette tips, vials, blades, etc.

Total library preparation cost for 100 samples: $256.41 £ \quad \sim 3.15$ \$ per sample
Cost for

100 samples

$390.00 £$ As listed at https://emea.illumina.com (March 2020)

$400.00 £$ Costs for quality control, data storage, etc. vary considerably among providers (quantity / price) $\mathbf{1 0 0}$ samples

1 run $/ 40.00 £ \quad 1$ run

9.72 \$ per sample; 70x MGRD expected based on 125x MGRD for 56 samples in run 2 


\section{References}

1. Schwabl, P. et al. Meiotic sex in Chagas disease parasite Trypanosoma cruzi. Nat. Commun. 10, (2019).

2. Guerra-Assunção, J. A. et al. Large-scale whole genome sequencing of M. tuberculosis provides insights into transmission in a high prevalence area. eLife 4, (2015).

3. Hall, M. D. et al. Improved characterisation of MRSA transmission using within-host bacterial sequence diversity. eLife 8, (2019).

4. Grigg, M. E., Bonnefoy, S., Hehl, A. B., Suzuki, Y. \& Boothroyd, J. C. Success and virulence in Toxoplasma as the result of sexual recombination between two distinct ancestries. Science 294, 161165 (2001).

5. $\mathrm{Wu}, \mathrm{Z}$. et al. Point mutations in the major outer membrane protein drive hypervirulence of a rapidly expanding clone of Campylobacter jejuni. Proc. Natl. Acad. Sci. USA 113, 10690-10695 (2016).

6. Miotto, O. et al. Genetic architecture of artemisinin-resistant Plasmodium falciparum. Nat. Genet. 47, 226-234 (2015).

7. Auburn, S. et al. Genomic analysis of a pre-elimination Malaysian Plasmodium vivax population reveals selective pressures and changing transmission dynamics. Nat. Commun. 9, (2018).

8. Teixeira, D. G. et al. Comparative analyses of whole genome sequences of Leishmania infantum isolates from humans and dogs in northeastern Brazil. Int. J. Parasitol. 47, 655-665 (2017).

9. Devera, R., Fernandes, O. \& Coura, J. R. Should Trypanosoma cruzi be called 'cruzi' complex? A review of the parasite diversity and the potential of selecting population after in vitro culturing and mice infection. Mem. Inst. Oswaldo Cruz 98, 1-12 (2003).

10. Alves, A. M., De Almeida, D. F. \& von Krüger, W. M. Changes in Trypanosoma cruzi kinetoplast DNA minicircles induced by environmental conditions and subcloning. J. Eukaryot. Microbiol. 41, 415-419 (1994).

11. Dvorak, J., Hartman, D. \& Miles, M. A. Trypanosoma cruzi: correlation of growth kinetics to zymodeme type in clones derived from various sources. J. Eukaryot. Microbiol. 27, 472-474 (2007). 
bioRxiv preprint doi: https://doi org/10.1101/2020.0324.003590; this version posted March 25,2020 . The copyright holder for this preprint (which was not certified by peer review) is the author/funder, who has granted bioRxiv a license to display the preprint in perpetuity. It is made available under aCC-BY-ND 4.0 International license.

12. Dean, M. P., Jansen, A. M., Mangia, R. H. R., Gonçalves, A. M. \& Morel C. M. Are our laboratory 'strains' representative samples of Trypanosoma cruzi populations that circulate in nature? Mem. Inst. Oswaldo Cruz 79, 19-24 (1984).

13. Lima, F. M. et al. Interclonal Variations in the molecular karyotype of Trypanosoma cruzi: chromosome rearrangements in a single cell-derived clone of the G strain. PLoS One $\mathbf{8}$, e63738 (2013).

14. Reis-Cunha, J. L. et al. Whole genome sequencing of Trypanosoma cruzi field isolates reveals extensive genomic variability and complex aneuploidy patterns within TcII DTU. BMC Genomics 19, $816(2018)$.

15. Cuypers, B. et al. Multiplexed spliced-leader sequencing: a high-throughput, selective method for RNA-seq in trypanosomatids. Sci. Rep. 7, 1-11 (2017).

16. Kumar, N. et al. Efficient subtraction of insect rRNA prior to transcriptome analysis of WolbachiaDrosophila lateral gene transfer. BMC Res. Notes 5, 230 (2012).

17. Oyola, S. O. et al. Efficient depletion of host DNA contamination in malaria clinical sequencing. $J$. Clin. Microbiol. 51, 745-751 (2013).

18. Feehery, G. R. et al. A method for selectively enriching microbial DNA from contaminating vertebrate host DNA. PLoS One 8, e76096 (2013).

19. Domagalska, M. A. et al. Genomes of intracellular Leishmania parasites directly sequenced from patients. bioRxiv 676163 (2019) doi:10.1101/676163.

20. Melnikov, A. et al. Hybrid selection for sequencing pathogen genomes from clinical samples. Genome Biol. 12, R73 (2011).

21. Schuenemann, V. J. et al. Genome-wide comparison of medieval and modern Mycobacterium leprae. Science 341, 179-183 (2013).

22. Metsky, H. C. et al. Zika virus evolution and spread in the Americas. Nature 546, 411-415 (2017).

23. Cowell, A. N. et al. Selective whole-genome amplification is a robust method that enables scalable whole-genome sequencing of Plasmodium vivax from unprocessed clinical samples. mBio 8, (2017).

24. Hintzsche, J. D., Robinson, W. A. \& Tan, A. C. A survey of computational tools to analyze and interpret whole exome sequencing data. Int. J. Genomics 2016, (2016). 
1018

25. Gampawar, P. et al. Evaluation of the performance of AmpliSeq and SureSelect exome sequencing libraries for Ion Proton. Front. Genet. 10, 856 (2019).

26. Nag, S. et al. High throughput resistance profiling of Plasmodium falciparum infections based on custom dual indexing and Illumina next generation sequencing-technology. Sci. Rep. 7, (2017).

27. Balkenhol, N., Cushman, S., Storfer, A. \& Waits, L. Landscape genetics: concepts, methods, applications. (John Wiley \& Sons, 2015).

28. Momčilović, S., Cantacessi, C., Arsić-Arsenijević, V., Otranto, D. \& Tasić-Otašević, S. Rapid diagnosis of parasitic diseases: current scenario and future needs. Clin. Microbiol. Infect. 25, 290309 (2019).

29. Arias, A. et al. Rapid outbreak sequencing of Ebola virus in Sierra Leone identifies transmission chains linked to sporadic cases. Virus Evol. 2, vew016 (2016).

30. Park, J. et al. Determining genotypic drug resistance by ion semiconductor sequencing with the Ion AmpliSeq ${ }^{\mathrm{TM}}$ TB Panel in multidrug-resistant Mycobacterium tuberculosis isolates. Ann. Lab. Med. 38, 316-323 (2018).

31. Ferrario, C. et al. A genome-based identification approach for members of the genus Bifidobacterium. FEMS Microbiol. Ecol. 91, (2015).

32. Makowsky, R. et al. Genomic diversity and phylogenetic relationships of human papillomavirus 16 (HPV16) in Nepal. Infect. Genet. Evol. 46, 7-11 (2016).

33. Grijalva, M. J., Suarez-Davalos, V., Villacis, A. G., Ocaña-Mayorga, S. \& Dangles, O. Ecological factors related to the widespread distribution of sylvatic Rhodnius ecuadoriensis populations in southern Ecuador. Parasit. Vectors 5, 17 (2012).

34. Nascimento, J. D. et al. Taxonomical over splitting in the Rhodnius prolixus (Insecta: Hemiptera: Reduviidae) clade: are $R$. taquarussuensis (da Rosa et al., 2017) and $R$. neglectus (Lent, 1954) the same species? PLoS One 14, e0211285 (2019).

35. Velásquez-Ortiz, N. et al. Trypanosoma cruzi infection, discrete typing units and feeding sources among Psammolestes arthuri (Reduviidae: Triatominae) collected in eastern Colombia. Parasit. Vectors 12, 157 (2019). 
36. Caicedo-Garzón, V. et al. Genetic diversification of Panstrongylus geniculatus (Reduviidae: Triatominae) in northern South America. PLoS One 14, (2019).

37. Carrasco, H. J., Torrellas, A., García, C., Segovia, M. \& Feliciangeli, M. D. Risk of Trypanosoma cruzi I (Kinetoplastida: Trypanosomatidae) transmission by Panstrongylus geniculatus (Hemiptera: Reduviidae) in Caracas (Metropolitan District) and neighboring states, Venezuela. Int. J. Parasitol. 35, 1379-1384 (2005).

38. Carrasco, H. J. et al. Geographical distribution of Trypanosoma cruzi genotypes in Venezuela. PLoS Negl. Trop. Dis. 6, (2012).

39. Nakad, B. C. C. et al. Genetic variability of Panstrongylus geniculatus (Reduviidae: Triatominae) in the Metropolitan District of Caracas, Venezuela. Infect. Genet. Evol. 66, 236-244 (2018).

40. Messenger, L. A., Yeo, M., Lewis, M. D., Llewellyn, M. S. \& Miles, M. A. Molecular genotyping of Trypanosoma cruzi for lineage assignment and population genetics. Methods Mol. Biol. 1201, 297-337 (2015).

41. Li, H. \& Durbin, R. Fast and accurate short read alignment with Burrows-Wheeler transform. Bioinformatics 25, 1754-1760 (2009).

42. DePristo, M. A. et al. A framework for variation discovery and genotyping using next-generation DNA sequencing data. Nat. Genet. 43, 491-498 (2011).

43. Derrien, T. et al. Fast computation and applications of genome mappability. PLoS One 7, (2012).

44. Franzén, O. et al. Comparative genomic analysis of human infective Trypanosoma cruzi lineages with the bat-restricted subspecies T. cruzi marinkellei. BMC Genomics 13, 531 (2012).

45. Li, L., Stoeckert, C. J. \& Roos, D. S. OrthoMCL: identification of ortholog groups for eukaryotic genomes. Genome Res. 13, 2178-2189 (2003).

46. Talavera-Lopez, C. et al. Repeat-driven generation of antigenic diversity in a major human pathogen, Trypanosoma cruzi. bioRxiv 283531 (2018) doi:10.1101/283531.

47. You, F. M. et al. BatchPrimer3: a high throughput web application for PCR and sequencing primer design. BMC Bioinformatics 9, 253 (2008).

48. Sonnhammer, E. L. \& Hollich, V. Scoredist : a simple and robust protein sequence distance estimator. BMC Bioinformatics 6, 108 (2005). 
1073

1074

1075

1076

1077

1078

1079

1080

1081

1082

1083

1084

1085

1086

1087

1088

1089

1090

1091

1092

1093

1094

1095

1096

1097

1098

1099

49. R: the R project for statistical computing. https://www.r-project.org/.

50. Cummings, K. L. \& Tarleton, R. L. Rapid quantitation of Trypanosoma cruzi in host tissue by realtime PCR. Mol. Biochem. Parasitol. 129, 53-59 (2003).

51. PhiX Sequencing Control V3. https://www.illumina.com/products/by-type/sequencing-kits/clustergen-sequencing-reagents/phix-control-v3.html.

52. Access Array System for Illumina Sequencing Systems (user guide). https://docplayer.net/78505463-Access-array-system-for-illumina-sequencing-systems.html.

53. Schmieder, R. \& Edwards, R. Fast identification and removal of sequence contamination from genomic and metagenomic datasets. PloS One 6, e17288 (2011).

54. Picard Tools. Broad Institute. http://broadinstitute.github.io/picard/.

55. Bandelt, H. J., Forster, P. \& Röhl, A. Median-joining networks for inferring intraspecific phylogenies. Mol. Biol. Evol. 16, 37-48 (1999).

56. Purcell, S. et al. PLINK: a tool set for whole-genome association and population-based linkage analyses. Am. J. Hum. Genet. 81, 559-575 (2007).

57. Ritland, K. Inferences about inbreeding depression based on changes of the inbreeding coefficient. Evolution 44, 1230-1241 (1990).

58. Wigginton, J. E., Cutler, D. J. \& Abecasis, G. R. A note on exact tests of Hardy-Weinberg equilibrium. Am. J. Hum. Genet. 76, 887-893 (2005).

59. Excoffier, L. \& Lischer, H. E. L. Arlequin suite ver 3.5: a new series of programs to perform population genetics analyses under Linux and Windows. Mol. Ecol. Resour. 10, 564-567 (2010).

60. Slatkin, M. A measure of population subdivision based on microsatellite allele frequencies. Genetics 139, 457-462 (1995).

61. Oksanen, J. et al. vegan: community ecology package.

62. Šavrič, B., Jenny, B. \& Jenny, H. Projection wizard - an online map projection selection tool. Cartogr. J. 53, 177-185 (2016).

63. Wiens, J. J. \& Morrill, M. C. Missing data in phylogenetic analysis: reconciling results from simulations and empirical data. Syst. Biol. 60, 719-731 (2011). 
bioRxiv preprint doi: https://doi org/10.1101/2020.0324.003590; this version posted March 25, 2020. The copyright holder for this preprint (which was not certified by peer review) is the author/funder, who has granted bioRxiv a license to display the preprint in perpetuity. It is made available under aCC-BY-ND 4.0 International license.

1100

1101

1102

1103

1104

1105

1106

1107

1108

1109

1110

1111

1112

1113

1114

1115

1116

1117

1118

1119

1120

1121

1122

1123

1124

1125

1126

64. Slatkin, M. Isolation by distance in equilibrium and non-equilibrium populations. Evol. Int. J. Org. Evol. 47, 264-279 (1993).

65. Zumaya-Estrada, F. A. et al. North American import? Charting the origins of an enigmatic Trypanosoma cruzi domestic genotype. Parasit. Vectors 5, 226 (2012).

66. Ocaña-Mayorga, S., Llewellyn, M. S., Costales, J. A., Miles, M. A. \& Grijalva, M. J. Sex, subdivision, and domestic dispersal of Trypanosoma cruzi lineage I in southern Ecuador. PLoS Negl. Trop. Dis. 4, e915 (2010).

67. Messenger, L. A. et al. Ecological host fitting of Trypanosoma cruzi TcI in Bolivia: mosaic population structure, hybridization and a role for humans in Andean parasite dispersal. Mol. Ecol. 24, 2406-2422 (2015).

68. Ramírez, J. D. et al. Contemporary cryptic sexuality in Trypanosoma cruzi. Mol. Ecol. 21, 42164226 (2012).

69. Llewellyn, M. S. et al. Trypanosoma cruzi IIc: phylogenetic and phylogeographic insights from sequence and microsatellite analysis and potential impact on emergent Chagas disease. PLoS Negl. Trop. Dis. 3, e510 (2009).

70. Roman, F. et al. Dissecting the phyloepidemiology of Trypanosoma cruzi I (TcI) in Brazil by the use of high resolution genetic markers. PLoS Negl. Trop. Dis. 12, e0006466 (2018).

71. Barnabe, C. et al. Putative panmixia in restricted populations of Trypanosoma cruzi isolated from wild Triatoma infestans in Bolivia. PloS One 8, e82269 (2013).

72. Llewellyn, M. S. The molecular epidemiology of Trypanosoma cruzi infection in wild and domestic transmission cycles with special emphasis on multilocus microsatellite analysis ( $\mathrm{PhD}$ thesis). London School of Hygiene \& Tropical Medicine (2008).

73. Shibata, H. et al. The use of PCR in detecting toxoplasma parasites in the blood and brains of mice experimentally infected with Toxoplasma gondii. Kansenshogaku Zasshi 69, 158-163 (1995).

74. Yang, H., Golenberg, E. M. \& Shoshani, J. Proboscidean DNA from museum and fossil specimens: an assessment of ancient DNA extraction and amplification techniques. Biochem. Genet. 35, 165179 (1997). 
75. Ramos, R. A. N. et al. Quantification of Leishmania infantum DNA in the bone marrow, lymph node and spleen of dogs. Rev. Bras. Parasitol. Vet. Braz. J. Vet. Parasitol. Orgao Of. Col. Bras. Parasitol. Vet. 22, 346-350 (2013).

76. Schubert, G. et al. Targeted detection of mammalian species using carrion fly - derived DNA. Mol. Ecol. Resour. 15, (2014).

77. Côté, N. M. L. et al. A new high-throughput approach to genotype ancient human gastrointestinal parasites. PLoS One 11, (2016).

78. Cencig, S., Coltel, N., Truyens, C. \& Carlier, Y. Parasitic loads in tissues of mice infected with Trypanosoma cruzi and treated with AmBisome. PLoS Negl. Trop. Dis. 5, (2011).

79. Souza, R. T. et al. Genome size, karyotype polymorphism and chromosomal evolution in Trypanosoma cruzi. PLoS One 6, e23042 (2011).

80. Reithinger, R., Lambson, B. E., Barker, D. C. \& Davies, C. R. Use of PCR to detect Leishmania (Viannia) spp. in dog blood and bone marrow. 38, 5 (2000).

81. Wen, C. et al. Evaluation of the reproducibility of amplicon sequencing with Illumina MiSeq platform. PLoS One 12, (2017).

82. Storfer, A., Patton, A. \& Fraik, A. K. Navigating the interface between landscape genetics and landscape genomics. Front. Genet. 9, (2018).

83. Erben, E. D. High-throughput methods for dissection of trypanosome gene regulatory networks. Curr. Genomics 19, 78-86 (2018).

84. Cingolani, P. et al. A program for annotating and predicting the effects of single nucleotide polymorphisms, SnpEff: SNPs in the genome of Drosophila melanogaster strain w1118; iso-2; iso3. Fly 6, 80-92 (2012).

85. Quinlan, A. R \& Hall, I. M. BEDTools: a flexible suite of utilities for comparing genomic features. Bioinformatics 26, 841- 842 (2010).

86. Aurrecoechea, C. et al. EuPathDB: the eukaryotic pathogen genomics database resource. Nucleic Acids Res. 45, D581-D591 (2017).

87. Danecek, P. et al. The variant call format and VCFtools. Bioinformatics 27, 2156-2158 (2011). 
bioRxiv preprint doi: https://doi.org/10.1101/2020 03.24.003590; this version posted March 25, 2020. The copyright holder for this preprint (which was not certified by peer review) is the author/funder, who has granted bioRxiv a license to display the preprint in perpetuity. It is made available under aCC-BY-ND 4.0 International license.

1154

1155

1156

1157

1158

1159

1160

1161

1162

1163

1164

1165

1166

1167

1168

1169

1170

1171

1172

1173

1174

1175

1176

1177

1178

1179

88. Linck, E. \& Battey, C. J. Minor allele frequency thresholds strongly affect population structure inference with genomic data sets. Mol. Ecol. Resour. 19, 639-647 (2019).

89. Excoffier, L., Dupanloup, I., Huerta-Sánchez, E., Sousa, V. C. \& Foll, M. Robust demographic inference from genomic and SNP data. PLoS Genet. 9, e1003905 (2013).

90. Bryant, D., Bouckaert, R., Felsenstein, J., Rosenberg, N. A. \& RoyChoudhury, A. Inferring species trees directly from biallelic genetic markers: bypassing gene trees in a full coalescent analysis. Mol. Biol. Evol. 29, 1917-1932 (2012).

91. Landguth, E. L., Bearlin, A., Day, C. C. \& Dunham, J. CDMetaPOP: an individual-based, ecoevolutionary model for spatially explicit simulation of landscape demogenetics. Methods Ecol. Evol. 8, 4-11 (2017).

92. Pritchard, J. K., Stephens, M. \& Donnelly, P. Inference of population structure using multilocus genotype data. Genetics 155, 945-959 (2000).

93. Piry, S. et al. GENECLASS2: a software for genetic assignment and first-generation migrant detection. J. Hered. 95, 536-539 (2004).

94. Cheng, L., Connor, T. R., Sirén, J., Aanensen, D. M. \& Corander, J. Hierarchical and spatially explicit clustering of DNA sequences with BAPS software. Mol. Biol. Evol. 30, 1224-1228 (2013).

95. Anderson, E. C. \& Thompson, E. A. A model-based method for identifying species hybrids using multilocus genetic data. Genetics 160, 1217-1229 (2002).

96. Graffelman, J., Jain, D. \& Weir, B. A genome-wide study of Hardy-Weinberg equilibrium with next generation sequence data. Hum. Genet. 136, 727-741 (2017).

97. Sefid Dashti, M. J. \& Gamieldien, J. A practical guide to filtering and prioritizing genetic variants. BioTechniques 62, 18-30 (2017).

98. Kaplinski, L., Andreson, R., Puurand, T. \& Remm, M. MultiPLX: automatic grouping and evaluation of PCR primers. Bioinformatics 21, 1701-1702 (2005).

99. Etherington, T. R. Python based GIS tools for landscape genetics: visualising genetic relatedness and measuring landscape connectivity. Methods Ecol. Evol. 2, 52-55 (2011). 
bioRxiv preprint doi: https://doi org/10.1101/2020.0324.003590; this version posted March 25, 2020. The copyright holder for this preprint (which was not certified by peer review) is the author/funder, who has granted bioRxiv a license to display the preprint in perpetuity. It is made available under aCC-BY-ND 4.0 International license.

100. Carrasco, H. J. et al. Panstrongylus geniculatus and four other species of triatomine bug involved in the Trypanosoma cruzi enzootic cycle: high risk factors for Chagas' disease transmission in the Metropolitan District of Caracas, Venezuela. Parasit. Vectors 7, 602 (2014).

101. Valadares, H. M. S. et al. Unequivocal identification of subpopulations in putative multiclonal Trypanosoma cruzi strains by FACs single cell sorting and genotyping. PLoS Negl. Trop. Dis. 6, e1722 (2012).

102. Zhu, S. J., Almagro-Garcia, J. \& McVean, G. Deconvolution of multiple infections in Plasmodium falciparum from high throughput sequencing data. Bioinformatics 34, 9-15 (2018).

103. Lerch, A. et al. Development of amplicon deep sequencing markers and data analysis pipeline for genotyping multi-clonal malaria infections. BMC Genomics 18, 864 (2017).

104. Chang, H.-H. et al. The real McCOIL: a method for the concurrent estimation of the complexity of infection and SNP allele frequency for malaria parasites. PLoS Comput. Biol. 13, (2017).

105. Hathaway, N. J., Parobek, C. M., Juliano, J. J. \& Bailey, J. A. SeekDeep: single-base resolution de novo clustering for amplicon deep sequencing. Nucleic Acids Res. 46, e21 (2018).

106. Zingales, B. Trypanosoma cruzi genetic diversity: something new for something known about Chagas disease manifestations, serodiagnosis and drug sensitivity. Acta Trop. 184, 38-52 (2018).

107. Nunes Maria Carmo Pereira et al. Chagas cardiomyopathy: an update of current clinical knowledge and management: a scientific statement from the American Heart Association. Circulation 138, e169-e209 (2018).

108. Llewellyn, M. S. et al. Extraordinary Trypanosoma cruzi diversity within single mammalian reservoir hosts implies a mechanism of diversifying selection. Int. J. Parasitol. 41, 609-614 (2011).

109. Pronovost, H. et al. Deep sequencing reveals multiclonality and new discrete typing units of Trypanosoma cruzi in rodents from the southern United States. J. Microbiol. Immunol. Infect. (2018).

110. Yeo, M. et al. Resolution of multiclonal infections of Trypanosoma cruzi from naturally infected triatomine bugs and from experimentally infected mice by direct plating on a sensitive solid medium. Int. J. Parasitol. 37, 111-120 (2007). 